\title{
Lewis Base Catalyzed, Enantioselective Aldol Addition of Methyl Trichlorosilyl Ketene Acetal to Ketones
}

\author{
Scott E. Denmark,* Yu Fan, and Martin D. Eastgate \\ Roger Adams Laboratory, Department of Chemistry, University of Illinois, \\ Urbana, IL 61801
}

\section{SUPPORTING INFORMATION}

Table of Contents

General Experimental

General Procedures

Optimization of Catalyzed Additions

Survey of Ketone Substrates

Preparation of $\mathrm{N}$-Oxides from Bipyridines

VT NMR and Crystallographic Studies

Computational Results

References and Stereochemical Assignments
Page

S1

S3

S6

$S 12$

S37

S52

S56

S78

\section{General Experimental}

All reactions were performed in oven $\left(140{ }^{\circ} \mathrm{C}\right)$ or flame-dried glassware under an inert atmosphere of dry $\mathrm{N}_{2}$. All reaction temperatures corresponded to internal temperature unless other wise noted. Solvents for extraction and chromatography were technical grade and distilled from the indicated drying agents: hexane $\left(\mathrm{CaCl}_{2}\right)$; methylene chloride $\left(\mathrm{CaCl}_{2}\right)$; ethyl acetate $\left(\mathrm{K}_{2} \mathrm{CO}_{3}\right)$. Reaction solvents were distilled from the indicated drying agents: methylene chloride $\left(\mathrm{P}_{2} \mathrm{O}_{5}\right)$; diethyl ether (sodium, benzophenone); tetrahydrofuran (THF) (sodium, benzophenone); and toluene (sodium). Diipropylethylamine was freshly distilled from $\mathrm{CaH}_{2}$. Flash column chromatography was performed using 230-400 mesh silica gel purchased from EM Science. 4'-(Trifluoromethyl)acetophenone, 4-methoxyacetophenone, 1-acetonaphthone, 2-bromo-5methylpyridine were used as received. Acetophenone, propiophenone, 2-acetylfuran, 2butanone, cyclopropyl methyl ketone, cyclohexyl methyl ketone, pinacolone, cyclohexenone, 4- 
phenyl-3-butyn-2-one, $\quad(E)$-4-phenyl-3-buten-2-one, $\quad 4$-phenyl-2-butanone, $\alpha$-tetralone, $\beta$ tetralone were freshly distilled before use. Methyl trichlorosilyl ketene acetal was prepared according to the literature procedure with modification. ${ }^{1}$ 1-Phenyl-2-propyn-1-one, ${ }^{2}(R, R)-6,6^{\prime}$ Bis-(1-methoxy-2,2-dimethylpropyl)-2,2'-bipyridine ${ }^{3}$ were prepared according to the literature procedures.

NMR spectra were recorded at $400 \mathrm{MHz}\left({ }^{1} \mathrm{H}\right) ; 100 \mathrm{MHz}\left({ }^{13} \mathrm{C}\right)$ and $376\left({ }^{19} \mathrm{~F}\right) \mathrm{MHz}$ or 500 $\mathrm{MHz}$ and $126 \mathrm{MHz}\left({ }^{13} \mathrm{C}\right) .{ }^{1} \mathrm{H}$ NMR and ${ }^{13} \mathrm{C} \mathrm{NMR}$ spectra are referenced to residual chloroform ( $\delta 7.26 \mathrm{ppm},{ }^{1} \mathrm{H} ; \delta 77.0 \mathrm{ppm},{ }^{13} \mathrm{C}$ ). ${ }^{19} \mathrm{~F}$ NMR spectra are referenced externally to $\mathrm{C}_{6} \mathrm{~F}_{6}$. Chemical

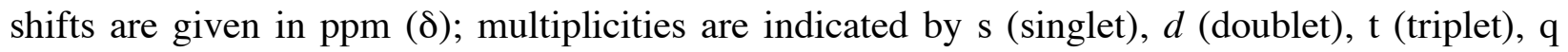
(quartet), m (multiplet) or br (broadened). Coupling constants, $J$, are reported in Hertz. All ${ }^{1} \mathrm{H}$ and ${ }^{13} \mathrm{C}$ NMR assignments are corroborated by 2D experiments (HETCOR and COSY). Spectra available on request from denmark@scs.uiuc.edu.

Low-resolution electron impact (EI) mass spectra were obtained on a with a typical ionization voltage of $70 \mathrm{eV}$. Data are reported in the form $\mathrm{m} / \mathrm{z}$ (intensity relative to base $=100$ ). Infrared spectra (IR) are reported in $\mathrm{cm}^{-1}$ with the following relative intensities: $\mathrm{s}$ (strong, 67100\%), m (medium, 34-66\%), w (weak, 0-33\%). Bulb-to-bulb distillations were done on a Kugelrohr apparatus; boiling points (bp) refer to air bath temperatures (ABT) and are uncorrected. Analytical TLC was performed on Merck silica gel plates with QF-254 indicator. Visualization was accomplished with potassium permanganate. Chiral stationary phase, supercritical fluid chromatography (CSP-SFC) was performed using Daicel Chiralpak columns with built-in photometric detector $(\lambda=220 \mathrm{~nm}$ or $258 \mathrm{~nm}) \mathrm{ns}$. Analytical capillary gas chromatography (GC) was performed with a flame ionization detection $\left(\mathrm{H}_{2}\right.$ carrier gas, 16 $\mathrm{mL} / \mathrm{min}$ ) using the following column : Astec (Chiraldex) BPH 30m x 0.25mm x 0.125 $\mu \mathrm{m}$. The injector temperature was $225{ }^{\circ} \mathrm{C}$, the detector temperature was $300{ }^{\circ} \mathrm{C}$. Analytical high pressure liquid chromatography (HPLC) was performed using a photometric detector $(\lambda=220 \mathrm{~nm}$ or 258 $\mathrm{nm})$ using a Rexchrom $(S, S) \beta$-Gem 1 column. Solvents for HPLC use were of spectroscopic grade and filtered before use. All separation methods for chiral samples were calibrated with racemic samples. Melting points $(\mathrm{mp})$ were determined on a capillary melting point apparatus and are uncorrected. Optical rotations were obtained on a digital polarimeter and are reported as follows: $[\alpha]_{D}^{T}$, concentration $(c=\mathrm{g} / 100 \mathrm{~mL})$, and solvent. 


\section{Experimental Procedures}

\section{Preparation of Methyl Tributylstannyl Acetate (4)}

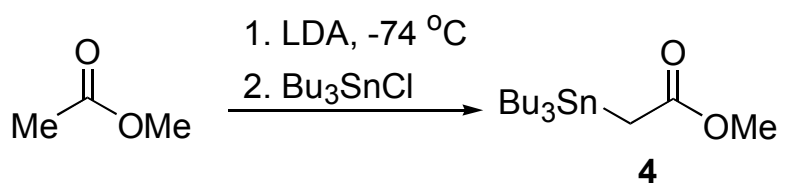

n-Butyllithium (124.0 mL, $1.52 \mathrm{M}$ in hexane, $222.0 \mathrm{mmol}, 1.2$ equiv) was added dropwise via a cannula to a stirred solution of diisopropylamine $(32.0 \mathrm{~mL}, \mathrm{~d}=0.742,222 \mathrm{mmol}$, 1.2 equiv) in dry THF $(1.0 \mathrm{~L})$ at 0 under nitrogen. The solution was cooled to $-74{ }^{\circ} \mathrm{C}$ and methyl acetate $\left(17.6 \mathrm{~mL}, \mathrm{~d}=0.932,222 \mathrm{mmol}, 1.2\right.$ equiv, distilled over $\left.\mathrm{K}_{2} \mathrm{CO}_{3}\right)$ in dry THF $(250 \mathrm{~mL})$ was added dropwise at a rate such that the internal temperature did not rise above $-70{ }^{\circ} \mathrm{C}$ (about $30 \mathrm{~min})$. The resulting solution was maintained between $-74{ }^{\circ} \mathrm{C}$ and $-70{ }^{\circ} \mathrm{C}$ for 30 min and then added via a short cannula over $10 \mathrm{~min}$ to tributyltin chloride $(50 \mathrm{~mL}, 184 \mathrm{mmol})$ in dry THF $\left(250 \mathrm{~mL}\right.$ ) also at $-74{ }^{\circ} \mathrm{C}$ under nitrogen. The mixture was warmed to $\mathrm{rt}$ over $2 \mathrm{~h}$ and stirred at $\mathrm{rt}$ for $30 \mathrm{~min}$. The mixture was concentrated under reduced pressure and the residue was taken up in hexane $(1.0 \mathrm{~L})$. A large amount of white precipitate $(\mathrm{LiCl}$ salt) was filtered off through Celite. The filtrate was concentrated under reduced pressure to provide $74.6 \mathrm{~g}$ of crude product $(110 \%)$ as light yellow oil, which was purified by preparative HPLC $\left(R_{f} 0.43\right.$, hexane/EtOAc $\left.12 / 1\right)$ using Waters PrePLC System 500A or alternatively at a smaller scale by flash column chromatography using triethylamine deactived silica gel. About $250 \mathrm{~g}$ of silica gel was loaded into a $50 \mathrm{~mm}$ column and was packed with a 90/10/1 mixture of hexane/EtOAc/triethylamine (1.2 L). The eluate was collected for running the column. From $14.3 \mathrm{~g}$ of crude product 4, $11.2 \mathrm{~g}$ was obtained after chromatography. The material was divided into two portions (about $6 \mathrm{~g}$ ) and bulbto-bulb distilled at $150{ }^{\circ} \mathrm{C}(0.1 \mathrm{~mm} \mathrm{Hg})$. It was found that the material without purification by silica gel column chromatography decomposed during distillation (ABT $150{ }^{\circ} \mathrm{C}, 0.1 \mathrm{~mm} \mathrm{Hg}$ ) using a short path distillation apparatus. On the other hand, analytically pure material (yields ranging from 60 to 82\%) was obtained using this two-step purification method. The spectroscopic data matched those from the literature. ${ }^{1}$ 


\section{Data for 4:}

bp: $\quad 150{ }^{\circ} \mathrm{C}$ ABT $(0.1 \mathrm{mmHg})$

TLC: $\quad R_{f} 0.43$ (hexane/EtOAc/triethylamine, 90/10/1)

Analysis: $\quad \mathrm{C}_{15} \mathrm{H}_{32} \mathrm{O}_{2} \mathrm{Sn}(363.11)$

Calcd: C, 49.61; H, 8.88\%

Found: $\quad$ C, 49.24; $\quad$ H, $9.12 \%$

\section{Preparation of Methyl Trichlorosilyl Ketene Acetal (1)}

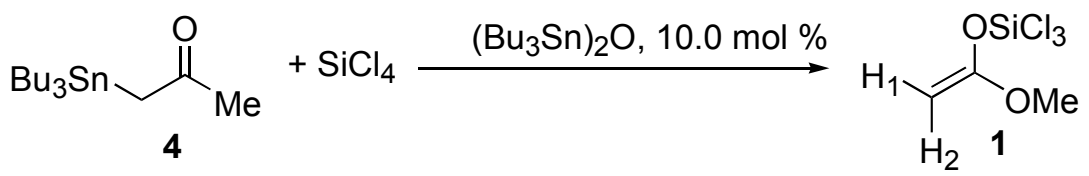

A. The apparatus for the preparation of 1: a one-piece room temperature distillation apparatus which consists of a short Vigreaux-column within a Claisen adapter, a dry-ice condenser and a receiving flask fitted with a septum is mounted on a $100 \mathrm{~mL}$ round-bottom flask with a septum and a magnetic stir bar.

B. Freshly distilled silicon tetrachloride $(10.0 \mathrm{~mL}, \mathrm{~d}=1.483,87.3 \mathrm{mmol}, 7.5$ equiv $)$ was loaded into a two-neck, $100 \mathrm{~mL}$ round bottom flask connected with a room temperature distillation apparatus consisting of a short. To rapidly stirred, neat $\mathrm{SiCl}_{4}$ was added dropwise 4 $(4.0 \mathrm{~mL}, \mathrm{~d}=1.063,11.7 \mathrm{mmol})$ over $10 \mathrm{~min}$, followed by $\left(\mathrm{Bu}_{3} \mathrm{Sn}\right)_{2} \mathrm{O}(0.6 \mathrm{~mL}, \mathrm{~d}=1.17,1.2$ mmol, 0.1 equiv). The mixture was stirred for $4 \mathrm{~h}$ at $\mathrm{rt}$ and the volatile was rapidly removed from the mixture under vacuum at $25{ }^{\circ} \mathrm{C}$ and condensed at $-78{ }^{\circ} \mathrm{C}$ (bath temperature). This mixture contained excess $\mathrm{SiCl}_{4}$ and the product $\mathbf{1}$ and it was transferred to another room temperature apparatus. The majority of $\mathrm{SiCl}_{4}$ was removed from the mixture at $25^{\circ} \mathrm{C}, 100 \mathrm{~mm}$ $\mathrm{Hg}$ and the product $1(2.01 \mathrm{~g}, 82 \%)$ was obtained after distillation at $25^{\circ} \mathrm{C}, 5 \mathrm{mmHg}$. A small portion of the product was reacted with excess of benzaldehyde. From the amount of the obtained aldol product the purity of 1 was determined to be $>90 \%$. ${ }^{1} \mathrm{H}$ NMR analysis indicated the presence of two impurities: the hydrolysis product of 1, methyl acetate, and the C-silyl isomer of $\mathbf{1}$, methyl trichlorosiylacetate.

It was found that $\mathbf{1}$ decomposed upon long exposure to tin containing by-products. Thus, 
the first distillation serves to separate 1 from the reaction mixture while the second distillation removes $\mathrm{SiCl}_{4}$ from 1 . Because in the first distillation full vacuum $(0.1 \mathrm{mmHg})$ was directly applied on a mixture of low boiling $\mathrm{SiCl}_{4}$ and $\mathbf{1}$, a relative large flask was used to avoid liquid "bumping". Methyl trichlorosilyl ketene acetal 1 gradually rearranges to its C-silyl isomer at rt, therefore it was stored in a freezer under argon. The spectroscopic data matched those from the literature. ${ }^{1}$

Data for 1:

bp: $\quad 25^{\circ} \mathrm{C}(5 \mathrm{mmHg})$

density: $\quad 1.3144 \mathrm{~g} / \mathrm{dm}^{3}$

${ }^{1}$ H NMR: $\quad\left(400 \mathrm{MHz}, \mathrm{CDCl}_{3}\right)$

$3.65(\mathrm{~d}, J=4.0,1 \mathrm{H}, \mathrm{H} 1) ; 3.64(\mathrm{~s}, 3 \mathrm{H}, \mathrm{OMe}) ; 3.41(\mathrm{~d}, J=3.9,1 \mathrm{H}, \mathrm{H} 2)$

\section{General Procedure I. Pyridine N-Oxide Catalyzed Aldol Reactions}

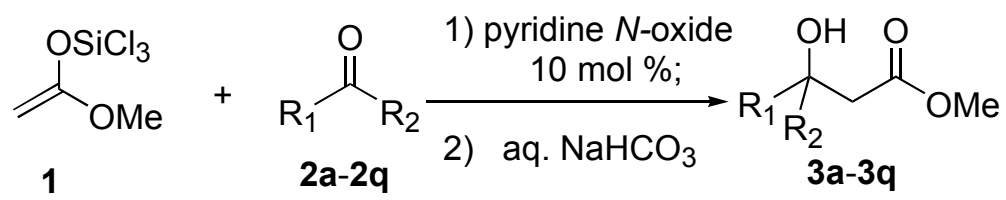

Trichlorosilyl ketene acetal 1 (1.2 equiv) was added to a solution of ketone (0.2 M) and pyridine $N$-oxide ( 0.1 equiv) in $\mathrm{CH}_{2} \mathrm{Cl}_{2}$ at $0^{\circ} \mathrm{C}$ or $\mathrm{rt}$ under nitrogen. After being stirred for the indicated time, the reaction mixture was transferred dropwise to an-ice-cold, saturated $\mathrm{NaHCO}_{3}$ solution $(20 \mathrm{~mL})$ with vigorous stirring. The mixture was further stirred for $30 \mathrm{~min}$ at $\mathrm{rt}$. The silicate precipitate was then filtered off through Celite and the filtrate was extracted by $\mathrm{CH}_{2} \mathrm{Cl}_{2}$ (4 x $20 \mathrm{~mL}$ ). The combined organic layers were dried over $\mathrm{MgSO}_{4}$ and concentrated under reduced pressure. Pyridine $N$-oxide was separated from the product by silica gel column chromatography (hexane/EtOAc). Analytically pure product was obtained after bulb-to-bulb distillation. 


\section{General Procedure II. Chiral Bipyridine Bis- $N$-Oxide Catalyzed Aldol Reactions}

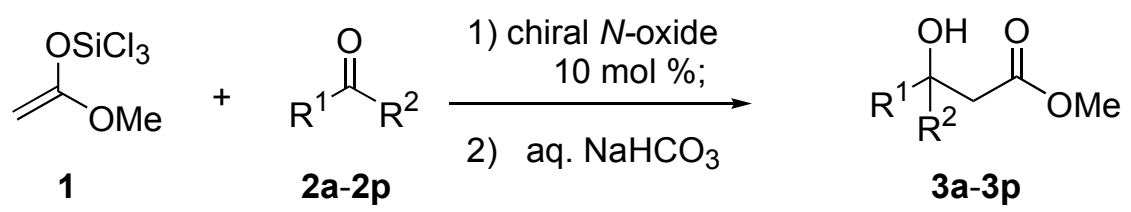

Trichlorosilyl ketene acetal 1 ( $2.4 \mathrm{mmol}, 1.2$ equiv) was added to a solution of ketone ( $2.0 \mathrm{mmol}$ ) and the indicated chiral bipyridine bis- $\mathrm{N}$-oxide (0.2 mmol, 0.1 equiv) in $\mathrm{CH}_{2} \mathrm{Cl}_{2}$ (10 $\mathrm{mL}$ ) at $-20{ }^{\circ} \mathrm{C}$ or $0{ }^{\circ} \mathrm{C}$ under nitrogen in a flame-dried, round-bottom flask with magnetic stirrer. After being stirred for the indicated time at $-20{ }^{\circ} \mathrm{C}$ or $0{ }^{\circ} \mathrm{C}$, the reaction mixture was transferred dropwise to an ice cold, saturated $\mathrm{NaHCO}_{3}$ solution $(20 \mathrm{~mL})$ with vigorous stirring. The mixture was further stirred for $30 \mathrm{~min}$ at $\mathrm{rt}$. The silicate precipitate was filtered off through Celite and the filtrate was extracted by $\mathrm{CH}_{2} \mathrm{Cl}_{2}(4 \times 20 \mathrm{~mL})$. The combined organic layers were dried over $\mathrm{MgSO}_{4}$ and concentrated under reduced pressure. The crude aldol product was separated from the catalyst by distillation and was further purified by silica gel chromatography. Analytically pure product was obtained after bulb-to-bulb distillation.

\section{Methyl 3-Hydroxy-3-phenylbutanoate (( \pm$)-3 a)$}

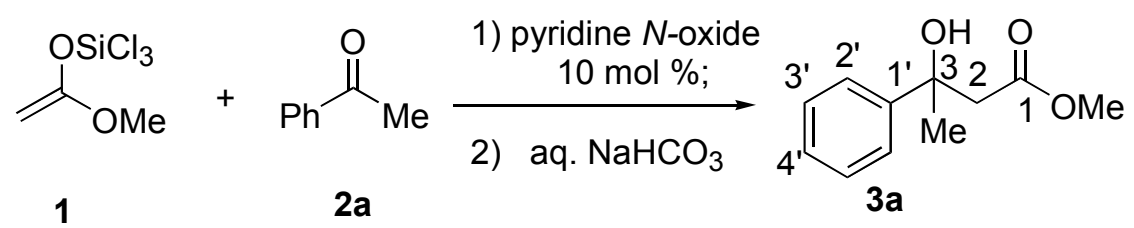

Following General Procedure I, a mixture of acetophenone (2a) (120 $\mu \mathrm{L}, 1.0 \mathrm{mmol})$, trichlorosilyl ketene acetal 1 (190 $\mu \mathrm{L}, 1.2 \mathrm{mmol}, 1.2$ equiv) and pyridine $N$-oxide (9.4 mg, 0.1 mmol, 0.1 equiv) in $\mathrm{CH}_{2} \mathrm{Cl}_{2}(5.0 \mathrm{~mL})$ was stirred at $\mathrm{rt}$ for $2 \mathrm{~h}$. Work-up provided $208 \mathrm{mg}$ of crude product. Analytically pure product (196 mg, 94\%) was obtained as a colorless liquid after bulb-to-bulb distillation. The spectroscopic data matched those from the literature. ${ }^{1}$ 


\section{Data for $( \pm)-3 \mathbf{a}:$}

bp: $\quad 110^{\circ} \mathrm{C}$ ABT $(1 \mathrm{mmHg})$

${ }^{1} \mathrm{H} \mathrm{NMR}: \quad\left(400 \mathrm{MHz}, \mathrm{CDCl}_{3}\right)$

7.24-7.45 (m, $5 \mathrm{H}$, Aryl); $4.35(\mathrm{~s}, 1 \mathrm{H}, \mathrm{OH}) ; 3.60\left(\mathrm{~s}, 3 \mathrm{H}, \mathrm{OCH}_{3}\right) ; 2.99(\mathrm{AB}$ quartet, $\left.J=15.9,2 \mathrm{H}, \mathrm{H}_{2} \mathrm{C}(2)\right) ; 1.53\left(\mathrm{~s}, 3 \mathrm{H}, \mathrm{H}_{3} \mathrm{C}\right)$

SFC: $(R)-3 \mathbf{a}, t_{\mathrm{R}} 2.79 \min (50.0 \%) ;(S)-3 \mathbf{a}, t_{\mathrm{R}} 3.04 \min (50.0 \%)$ (Chiracel OJ, $1.1 \%$ $\mathrm{MeOH}$ in $\mathrm{CO}_{2}, 150$ bar, $40{ }^{\circ} \mathrm{C}, 2.5 \mathrm{~mL} \mathrm{~min}^{-1}$ )

Table 6, entry 5. Following General Procedure II, acetophenone (2a) (60 $\mu \mathrm{L}, 0.5$ mmol), trichlorosilyl ketene acetal $1(100 \mu \mathrm{L}, 0.6 \mathrm{mmol}, 1.2$ equiv) and bis- $N$-oxide $(R, R)-17(20$ $\mathrm{mg}, 0.05 \mathrm{mmol}, 0.1$ equiv) were stirred at $0{ }^{\circ} \mathrm{C}$ for $3 \mathrm{~h}$. Work-up provided $80 \mathrm{mg}(92 \%)$ of product.

SFC: $(R)-3 a, t_{\mathrm{R}} 2.89 \min (22.6 \%) ;(S)-3 a, t_{\mathrm{R}} 3.10 \min (77.4 \%)$ (Chiracel OJ, 1.1\% $\mathrm{MeOH}$ in $\mathrm{CO}_{2}, 150$ bar, $40{ }^{\circ} \mathrm{C}, 2.5 \mathrm{~mL} \mathrm{~min}^{-1}$ )

Table 6, entry 6. Following General Procedure II, acetophenone (3a) (60 $\mu \mathrm{L}, 0.5$ mmol), trichlorosilyl ketene acetal $1(100 \mu \mathrm{L}, 0.6 \mathrm{mmol}, 1.2$ equiv) and bis- $N$-oxide $(R, R)-18$ (24 mg, $0.05 \mathrm{mmol}, 0.1$ equiv) were stirred at $0{ }^{\circ} \mathrm{C}$ for $3 \mathrm{~h}$. Work-up provided $82 \mathrm{mg}(94 \%$ ) of product.

SFC: $\quad(R)-3 \mathbf{a}, t_{\mathrm{R}} 2.79 \min (18.1 \%) ;(S)-3 \mathbf{a}, t_{\mathrm{R}} 3.00 \min (81.9 \%)$ (Chiracel OJ, $1.1 \%$ $\mathrm{MeOH}$ in $\mathrm{CO}_{2}, 150$ bar, $40{ }^{\circ} \mathrm{C}, 2.5 \mathrm{~mL} \mathrm{~min}^{-1}$ )

Table 7, entry 1. Following General Procedure II, acetophenone (2a) $(60 \mu \mathrm{L}, 0.5$ mmol), trichlorosilyl ketene acetal $1(100 \mu \mathrm{L}, 0.6 \mathrm{mmol}, 1.2$ equiv) and bis- $N$-oxide $(P)-(R, R)-19$ (25 mg, $0.05 \mathrm{mmol}, 0.1$ equiv) were stirred at $0{ }^{\circ} \mathrm{C}$ for $3 \mathrm{~h}$. Work-up provided $78 \mathrm{mg}(90 \%)$ of product.

SFC: $(R)-3 a, t_{\mathrm{R}} 2.86 \min (12.9 \%) ;(S)-3 \mathbf{a}, t_{\mathrm{R}} 3.07 \min (87.1 \%)$ (Chiracel OJ, $1.1 \%$ $\mathrm{MeOH}$ in $\mathrm{CO}_{2}, 150$ bar, $40{ }^{\circ} \mathrm{C}, 2.5 \mathrm{~mL} \mathrm{~min}^{-1}$ ) 
Table 7, entry 2. Following General Procedure II, acetophenone (2a) (240 $\mu \mathrm{L}, 2.0$ mmol), trichlorosilyl ketene acetal $1(380 \mu \mathrm{L}, 2.4 \mathrm{mmol}, 1.2$ equiv) and bis- $N$-oxide $(P)-(R, R)-19$ (101 mg, $0.2 \mathrm{mmol}, 0.1$ equiv) were stirred at $-20{ }^{\circ} \mathrm{C}$ for $12 \mathrm{~h}$. Work-up provided $478 \mathrm{mg}$ of crude mixture. Analytically pure product (364 mg, 94\%) was obtained as a colorless liquid after bulb-to-bulb distillation. From the sign of optical rotation of the product $\left([\alpha]_{\mathrm{D}}^{24}-5.62(\mathrm{EtOH})\right)$, the absolute configuration of $\mathbf{3 a}$ was deduced to be $S$ (lit. ${ }^{4 a}, \mathbf{3 a}$, er $S / R 79 / 31,[\alpha]_{\mathrm{D}}^{24}-4.96$ $(\mathrm{EtOH}))$.

Data for $(S)-\mathbf{3 a}$ :

SFC: $(R)-3 \mathbf{a}, t_{\mathrm{R}} 2.85 \min (8.7 \%)$; $(S)-3 \mathbf{a}, t_{\mathrm{R}} 3.04 \min (91.2 \%)$ (Chiracel OJ, $1.1 \%$ $\mathrm{MeOH}$ in $\mathrm{CO}_{2}, 150$ bar, $40{ }^{\circ} \mathrm{C}, 2.5 \mathrm{~mL} \mathrm{~min}^{-1}$ )

Opt. Rot.: $[\alpha]_{\mathrm{D}}^{24}-5.6(c=1.09, \mathrm{EtOH})$

Analysis: $\quad \mathrm{C}_{11} \mathrm{H}_{14} \mathrm{O}_{3}(194.22)$

$\begin{array}{lll}\text { Calcd: } & \mathrm{C}, 68.02 ; & \mathrm{H}, 7.26 \% \\ \text { Found: } & \mathrm{C}, 67.69 ; & \mathrm{H}, 7.35 \%\end{array}$

Table 7, entry 3. Following General Procedure II, acetophenone (2a) (60 $\mu \mathrm{L}, 0.5$ mmol), trichlorosilyl ketene acetal $1(100 \mu \mathrm{L}, 0.6 \mathrm{mmol}, 1.2$ equiv) and bis- $N$-oxide $(M)$ - $(R, R)$ 19 (25 mg, $0.05 \mathrm{mmol}, 0.1$ equiv) were stirred at $-20{ }^{\circ} \mathrm{C}$ for $12 \mathrm{~h}$. Work-up provided $77 \mathrm{mg}$ $(89 \%)$ of product.

SFC: $(R)-3 a, t_{\mathrm{R}} 2.81 \min (71.4 \%) ;(S)-3 \mathbf{a}, t_{\mathrm{R}} 3.04 \min (29.6 \%)$ (Chiracel OJ, $1.1 \%$ $\mathrm{MeOH}$ in $\mathrm{CO}_{2}, 150$ bar, $40{ }^{\circ} \mathrm{C}, 2.5 \mathrm{~mL} \mathrm{~min}^{-1}$ )

Table 7, entry 4. Following General Procedure II, acetophenone (2a) (60 $\mu \mathrm{L}, 0.5$ mmol), trichlorosilyl ketene acetal $1(100 \mu \mathrm{L}, 0.6 \mathrm{mmol}, 1.2$ equiv) and bis- $N$-oxide $(P)-(R, R)-20$ (33 mg, $0.05 \mathrm{mmol}, 0.1$ equiv) were stirred at $-20{ }^{\circ} \mathrm{C}$ for $12 \mathrm{~h}$. Work-up provided $77 \mathrm{mg}(89 \%)$ of product.

SFC: $(R)-3 \mathbf{a}, t_{\mathrm{R}} 2.81 \min (9.0 \%) ;(S)-3 \mathbf{a}, t_{\mathrm{R}} 3.04 \min (91.0 \%)$ (Chiracel OJ, $1.1 \%$ $\mathrm{MeOH}$ in $\mathrm{CO}_{2}, 150$ bar, $40{ }^{\circ} \mathrm{C}, 2.5 \mathrm{~mL} \mathrm{~min}^{-1}$ ) 
Table 7, entry 5. Following General Procedure II, acetophenone (2a) (60 $\mu \mathrm{L}, 0.5$ mmol), trichlorosilyl ketene acetal $1(100 \mu \mathrm{L}, 0.6 \mathrm{mmol}, 1.2$ equiv) and bis- $N$-oxide $(P)-(R, R)-21$ (38 mg, $0.05 \mathrm{mmol}, 0.1$ equiv) were stirred at $-20{ }^{\circ} \mathrm{C}$ for $12 \mathrm{~h}$. Work-up provided $79 \mathrm{mg}(91 \%)$ of product.

SFC: $(R)-3 \mathbf{a}, t_{\mathrm{R}} 2.81 \min (6.5 \%) ;(S)-3 \mathbf{a}, t_{\mathrm{R}} 3.04 \min (93.5 \%)$ (Chiracel OJ, $1.1 \%$ $\mathrm{MeOH}$ in $\mathrm{CO}_{2}, 150$ bar, $40{ }^{\circ} \mathrm{C}, 2.5 \mathrm{~mL} \mathrm{~min}^{-1}$ )

Table 7, entry 6. Following General Procedure II, acetophenone (2a) (60 $\mu \mathrm{L}, 0.5$ mmol), trichlorosilyl ketene acetal $1(100 \mu \mathrm{L}, 0.6 \mathrm{mmol}, 1.2$ equiv) and bis- $N$-oxide $(P)-(R, R)-22$ (42 mg, $0.05 \mathrm{mmol}, 0.1$ equiv) were stirred at $-20{ }^{\circ} \mathrm{C}$ for $12 \mathrm{~h}$. Work-up provided $57 \mathrm{mg}(64 \%$ ) of product.

SFC: $(R)-3 a, t_{\mathrm{R}} 2.81 \mathrm{~min}(20.0 \%) ;(S)-3 \mathbf{a}, t_{\mathrm{R}} 3.04 \mathrm{~min}(80.0 \%)$ (Chiracel OJ, $1.1 \%$ $\mathrm{MeOH}$ in $\mathrm{CO}_{2}, 150$ bar, $40{ }^{\circ} \mathrm{C}, 2.5 \mathrm{~mL} \mathrm{~min}^{-1}$ )

Table 7, entry 7. Following General Procedure II, acetophenone (2a) (60 $\mu \mathrm{L}, 0.5$ mmol), trichlorosilyl ketene acetal $1(100 \mu \mathrm{L}, 0.6 \mathrm{mmol}, 1.2$ equiv) and bis- $N$-oxide $(P)-(R, R)-\mathbf{2 3}$ (33 mg, $0.05 \mathrm{mmol}, 0.1$ equiv) were stirred at $-20{ }^{\circ} \mathrm{C}$ for $12 \mathrm{~h}$. Work-up provided $76 \mathrm{mg}(88 \%)$ of product.

SFC: $\quad(R)-3 \mathbf{a}, t_{\mathrm{R}} 2.81 \min (13.0 \%) ;(S)-3 \mathbf{a}, t_{\mathrm{R}} 3.04 \min (87.0 \%)$ (Chiracel OJ, $1.1 \%$ $\mathrm{MeOH}$ in $\mathrm{CO}_{2}, 150$ bar, $40{ }^{\circ} \mathrm{C}, 2.5 \mathrm{~mL} \mathrm{~min}^{-1}$ )

Table 7, entry 8. Following General Procedure II, acetophenone (2a) (60 $\mu \mathrm{L}, 0.5$ mmol), trichlorosilyl ketene acetal $1(100 \mu \mathrm{L}, 0.6 \mathrm{mmol}, 1.2$ equiv) and bis- $N$-oxide $(P)-(R, R)-24$ (33 mg, $0.05 \mathrm{mmol}, 0.1$ equiv) were stirred at $-20{ }^{\circ} \mathrm{C}$ for $12 \mathrm{~h}$. Work-up provided $79 \mathrm{mg}(91 \%)$ of product.

SFC: $(R)-3 \mathbf{a}, t_{\mathrm{R}} 2.81 \min (49.5 \%) ;(S)-3 \mathbf{a}, t_{\mathrm{R}} 3.04 \min (50.5 \%)$ (Chiracel OJ, $1.1 \%$ $\mathrm{MeOH}$ in $\mathrm{CO}_{2}, 150$ bar, $40{ }^{\circ} \mathrm{C}, 2.5 \mathrm{~mL} \mathrm{~min}^{-1}$ ) 
Table 7, entry 9. Following General Procedure II, acetophenone (2a) (60 $\mu \mathrm{L}, 0.5$ mmol), trichlorosilyl ketene acetal $1(100 \mu \mathrm{L}, 0.6 \mathrm{mmol}, 1.2$ equiv) and bis- $N$-oxide $(P)-(R, R)-25$ (24 mg, $0.05 \mathrm{mmol}, 0.1$ equiv) were stirred at $-20{ }^{\circ} \mathrm{C}$ for $12 \mathrm{~h}$. Work-up provided $81 \mathrm{mg}(93 \%)$ of product.

SFC: $(R)-3 a, t_{\mathrm{R}} 2.81 \mathrm{~min}(18.0 \%) ;(S)-3 \mathbf{a}, t_{\mathrm{R}} 3.04 \mathrm{~min}(82.0 \%)$ (Chiracel OJ, $1.1 \%$ $\mathrm{MeOH}$ in $\mathrm{CO}_{2}, 150$ bar, $40{ }^{\circ} \mathrm{C}, 2.5 \mathrm{~mL} \mathrm{~min}^{-1}$ )

Table 7, entry 10. Following General Procedure II, acetophenone (2a) (60 $\mu \mathrm{L}, 0.5$ mmol), trichlorosilyl ketene acetal $1(100 \mu \mathrm{L}, 0.6 \mathrm{mmol}, 1.2$ equiv) and bis- $N$-oxide $(P)-(R, R)-26$ ( $25 \mathrm{mg}, 0.05 \mathrm{mmol}, 0.1$ equiv) were stirred at $-20{ }^{\circ} \mathrm{C}$ for $12 \mathrm{~h}$. Work-up provided $78 \mathrm{mg}(90 \%)$ of product.

SFC: $(R)-3 a, t_{\mathrm{R}} 2.81 \min (10.1 \%) ;(S)-3 \mathbf{a}, t_{\mathrm{R}} 3.04 \mathrm{~min}(89.9 \%)$ (Chiracel OJ, $1.1 \%$ $\mathrm{MeOH}$ in $\mathrm{CO}_{2}, 150$ bar, $40{ }^{\circ} \mathrm{C}, 2.5 \mathrm{~mL} \mathrm{~min}^{-1}$ )

Table 7, entry 11. Following General Procedure II, acetophenone (2a) (60 $\mu \mathrm{L}, 0.5$ mmol), trichlorosilyl ketene acetal $1(100 \mu \mathrm{L}, 0.6 \mathrm{mmol}, 1.2$ equiv) and bis- $N$-oxide $(P)-(R, R)-27$ (38 mg, $0.05 \mathrm{mmol}, 0.1$ equiv) were stirred at $-20{ }^{\circ} \mathrm{C}$ for $12 \mathrm{~h}$. Work-up provided $75 \mathrm{mg}(87 \%)$ of product.

SFC: $\quad(R)-3 a, t_{\mathrm{R}} 2.81 \min (26.5 \%) ;(S)-3 \mathbf{a}, t_{\mathrm{R}} 3.04 \min (73.5 \%)$ (Chiracel OJ, $1.1 \%$ $\mathrm{MeOH}$ in $\mathrm{CO}_{2}, 150$ bar, $40{ }^{\circ} \mathrm{C}, 2.5 \mathrm{~mL} \mathrm{~min}^{-1}$ )

Table 7, entry 12. Following General Procedure II, acetophenone (2a) (60 $\mu \mathrm{L}, 0.5$ mmol), trichlorosilyl ketene acetal $1(100 \mu \mathrm{L}, 0.6 \mathrm{mmol}, 1.2$ equiv) and bis- $N$-oxide $(P)-(R, R)-28$ (31 mg, $0.05 \mathrm{mmol}, 0.1$ equiv) were stirred at $-20{ }^{\circ} \mathrm{C}$ for $12 \mathrm{~h}$. Work-up provided $23 \mathrm{mg}(32 \%$ ) of product.

SFC: $\quad(R)-3 \mathbf{a}, t_{\mathrm{R}} 2.81 \min (19.5 \%) ;(S)-3 \mathbf{a}, t_{\mathrm{R}} 3.04 \min (80.5 \%)$ (Chiracel OJ, $1.1 \%$ $\mathrm{MeOH}$ in $\mathrm{CO}_{2}, 150$ bar, $40{ }^{\circ} \mathrm{C}, 2.5 \mathrm{~mL} \mathrm{~min}^{-1}$ ) 
Table 7, entry 13. Following General Procedure II, acetophenone (2a) (60 $\mu \mathrm{L}, 0.5$ mmol), trichlorosilyl ketene acetal $1(100 \mu \mathrm{L}, 0.6 \mathrm{mmol}, 1.2$ equiv) and bis- $N$-oxide $(P)-(R, R)-29$ (27 mg, $0.05 \mathrm{mmol}, 0.1$ equiv) were stirred at $-20{ }^{\circ} \mathrm{C}$ for $12 \mathrm{~h}$. Work-up provided $83 \mathrm{mg}(95 \%$ ) of product.

SFC: $(R)-3 a, t_{\mathrm{R}} 2.81 \mathrm{~min}(14.0 \%) ;(S)-3 \mathbf{a}, t_{\mathrm{R}} 3.04 \mathrm{~min}(86.0 \%)$ (Chiracel OJ, $1.1 \%$ $\mathrm{MeOH}$ in $\mathrm{CO}_{2}, 150$ bar, $40{ }^{\circ} \mathrm{C}, 2.5 \mathrm{~mL} \mathrm{~min}^{-1}$ )

Table 7, entry 14. Following General Procedure II, acetophenone (2a) (60 $\mu \mathrm{L}, 0.5$ mmol), trichlorosilyl ketene acetal $1(100 \mu \mathrm{L}, 0.6 \mathrm{mmol}, 1.2$ equiv) and bis- $N$-oxide $(P)-(R, R)-30$ (27 mg, $0.05 \mathrm{mmol}, 0.1$ equiv) were stirred at $-20{ }^{\circ} \mathrm{C}$ for $12 \mathrm{~h}$. Work-up provided $81 \mathrm{mg}(93 \%)$ of product.

SFC: $(R)-3 a, t_{\mathrm{R}} 2.81 \mathrm{~min}(14.0 \%) ;(S)-3 \mathbf{a}, t_{\mathrm{R}} 3.04 \mathrm{~min}(86.0 \%)$ (Chiracel OJ, $1.1 \%$ $\mathrm{MeOH}$ in $\mathrm{CO}_{2}, 150$ bar, $40{ }^{\circ} \mathrm{C}, 2.5 \mathrm{~mL} \mathrm{~min}^{-1}$ )

Table 7, entry 15. Following General Procedure II, acetophenone (2a) (60 $\mu \mathrm{L}, 0.5$ mmol), trichlorosilyl ketene acetal $1(100 \mu \mathrm{L}, 0.6 \mathrm{mmol}, 1.2$ equiv) and bis- $N$-oxide $31 \mathrm{~A}$ ( $24 \mathrm{mg}$, $0.05 \mathrm{mmol}, 0.1$ equiv) were stirred at $-20{ }^{\circ} \mathrm{C}$ for $12 \mathrm{~h}$. Work-up provided $81 \mathrm{mg}(92 \%)$ of product.

SFC: $\quad(R)-3 \mathbf{a}, t_{\mathrm{R}} 2.81 \min (38.0 \%) ;(S)-3 \mathbf{a}, t_{\mathrm{R}} 3.04 \min (62.0 \%)$ (Chiracel OJ, $1.1 \%$ $\mathrm{MeOH}$ in $\mathrm{CO}_{2}, 150$ bar, $40{ }^{\circ} \mathrm{C}, 2.5 \mathrm{~mL} \mathrm{~min}^{-1}$ )

Table 7, entry 16. Following General Procedure II, acetophenone (2a) (60 $\mu \mathrm{L}, 0.5$ mmol), trichlorosilyl ketene acetal 1 (100 $\mu \mathrm{L}, 0.6 \mathrm{mmol}, 1.2$ equiv) and bis- $N$-oxide $31 \mathbf{B}$ (27 mg, $0.05 \mathrm{mmol}, 0.1$ equiv) were stirred at $-20{ }^{\circ} \mathrm{C}$ for $12 \mathrm{~h}$. Work-up provided $82 \mathrm{mg}$ (94\%) of product.

SFC: $(R)-3 \mathbf{a}, t_{\mathrm{R}} 2.81 \min (52.5 \%) ;(S)-3 \mathbf{a}, t_{\mathrm{R}} 3.04 \min (47.5 \%)$ (Chiracel OJ, $1.1 \%$ $\mathrm{MeOH}$ in $\mathrm{CO}_{2}, 150$ bar, $40{ }^{\circ} \mathrm{C}, 2.5 \mathrm{~mL} \mathrm{~min}^{-1}$ ) 
Methyl 3-Hydroxy-3-phenyl-pentanoate (( \pm -3b)

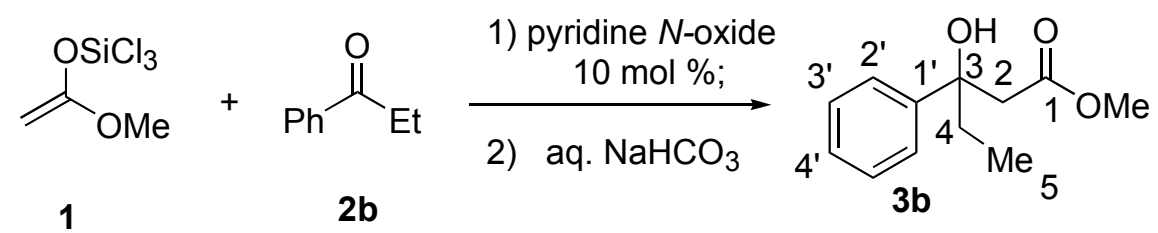

Following General Procedure I, propiophenone (2b) (132 $\mu \mathrm{L}, 1.0 \mathrm{mmol})$, trichlorosilyl ketene acetal $1(190 \mu \mathrm{L}, 1.2 \mathrm{mmol}, 1.2$ equiv) and pyridine $N$-oxide (9.6 mg, $0.1 \mathrm{mmol}, 0.1$ equiv) were stirred at $\mathrm{rt}$ for $2 \mathrm{~h}$. Work-up provided $213 \mathrm{mg}$ of crude product. Analytically pure product (191 mg, 92\%) was obtained as a white solid after bulb-to-bulb distillation.

Data for $( \pm)-\mathbf{3 b}$ :

bp: $\quad 110^{\circ} \mathrm{C} \operatorname{ABT}(0.2 \mathrm{mmHg})$

mp: $\quad 50-50.5^{\circ} \mathrm{C}$

${ }^{1} \mathrm{H}$ NMR: $\quad\left(500 \mathrm{MHz}, \mathrm{CDCl}_{3}\right)$

7.22-7.40 (m, $5 \mathrm{H}, \mathrm{Ar})$; (bs, $1 \mathrm{H}, \mathrm{OH}) ; 3.57$ (s, $\left.3 \mathrm{H}, \mathrm{OCH}_{3}\right) ; 2.88$ (AB quartet, $J=$ 15.6, $\left.2 \mathrm{H}, \mathrm{H}_{2} \mathrm{C}(2)\right) ; 1.751 .86\left(\mathrm{~m}, 2 \mathrm{H}, \mathrm{H}_{2} \mathrm{C}(4)\right)$; 0.76 (t, $\left.J=7.3,3 \mathrm{H}, \mathrm{H}_{3} \mathrm{C}(5)\right)$

${ }^{13} \mathrm{C}$ NMR: $\quad\left(100 \mathrm{MHz}, \mathrm{CDCl}_{3}\right)$

$173.4(\mathrm{C}(1)), 145.1\left(\mathrm{C}\left(1^{\prime}\right)\right), 129.1\left(\mathrm{C}\left(3^{\prime}\right)\right), 126.7\left(\mathrm{C}\left(4^{\prime}\right)\right), 125.1\left(\mathrm{C}\left(2^{\prime}\right)\right), 75.1(\mathrm{C}(3))$,

51.7 (C(OMe)), 44.7 (C(2)), 35.9 (C(4)), 7.8 (C(5))

IR: (neat)

3515 (m), 3021 (s), 2939 (m), 1716 (s), 1440 (s), 1347 (m), 1210 (m), 1174 (m)

TLC: $\quad R_{f} 0.30$ (hexane/EtOAc, 5/1)

GC: $\quad(S)-3 b, t_{\mathrm{R}} 7.07 \min (49.9 \%) ;(R)-3 \mathbf{b}, t_{\mathrm{R}} 7.35 \min (50.1 \%)$ (Chiraldex GTA, 115

$\left.{ }^{\circ} \mathrm{C}, 18 \mathrm{psi}\right)$

MS: $\quad(\mathrm{FI}, 70 \mathrm{eV})$

208 (27), 180 (12), 179 (100)

Analysis: $\quad \mathrm{C}_{12} \mathrm{H}_{16} \mathrm{O}_{3}(220.27)$

Calcd: $\quad$ C, 69.21; $\quad \mathrm{H}, 7.74 \%$

Found: $\quad$ C, 69.08; H, 7.80\% 
Following General Procedure II, propiophenone (2b) $(264 \mu \mathrm{L}, 2.0 \mathrm{mmol})$, trichlorosilyl ketene acetal 3b (380 $\mu \mathrm{L}, 2.4 \mathrm{mmol}, 1.2$ equiv) and bis- $N$-oxide $(P)-(R, R)$-19 (101 mg, 0.2 mmol, 0.1 equiv) were stirred at $-20{ }^{\circ} \mathrm{C}$ for $12 \mathrm{~h}$. Work-up provided $560 \mathrm{mg}$ of crude product. Analytically pure product (395 mg, 94\%) was obtained as a colorless liquid after bulb-to-bulb distillation. From the sign of optical rotation of the corresponding carboxylic acid, $\left([\alpha]_{D}^{24}+16.0\right.$ $(\mathrm{EtOH})$ ), the absolute configuration of $\mathbf{3 b}$ was deduced to be $S$ (lit..,$(S)$-enantiomer of 3hydroxy-3-phenylpentanoic acid, $\left.[\alpha]_{\mathrm{D}}^{24}+22.0(\mathrm{EtOH})\right)$.

Data for $(+)-3 \mathbf{b}$ :

GC: $\quad(S)-3 b, t_{\mathrm{R}} 7.08 \min (90.3 \%) ;(R)-3 b, t_{\mathrm{R}} 7.36 \min (9.7 \%)$ (Chiraldex GTA, $115{ }^{\circ} \mathrm{C}$, $18 \mathrm{psi})$

Opt. Rot.: $[\alpha]_{\mathrm{D}}^{24}+16.0(c=0.62, \mathrm{EtOH})$ (of the corresponding carboxylic acid)

Analysis: $\quad \mathrm{C}_{12} \mathrm{H}_{16} \mathrm{O}_{3}(220.27)$
Calcd:
C, 69.21;
$\mathrm{H}, 7.74 \%$
Found:
C, 69.23;
H, $7.79 \%$ 
Methyl 3-Hydroxy-3-phenyl-4-pentynoate ((土)-3c)

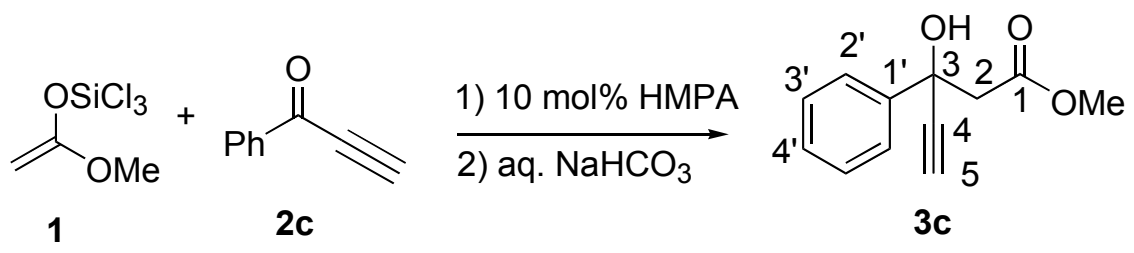

Following General Procedure I, 1-phenyl-2-pentyn-1-one (2c) (130 mg, $1.0 \mathrm{mmol}$ ), trichlorosilyl ketene acetal 1 (190 $\mu \mathrm{L}, 1.2 \mathrm{mmol}, 1.2$ equiv) and HMPA (9 $\mu \mathrm{L}, 0.1 \mathrm{mmol}, 0.1$ equiv) were stirred at $\mathrm{rt}$ for $2 \mathrm{~h}$. Work-up provided $221 \mathrm{mg}$ of crude product which was purified by silica gel chromatography (hexane/EtOAc, 10/1). Analytically pure product (147 mg, 71\%) was obtained as colorless oil after bulb-to-bulb distillation.

Data for $( \pm)-\mathbf{3 c}$ :

bp: $\quad 110^{\circ} \mathrm{C}$ ABT $(0.2 \mathrm{mmHg})$

${ }^{1} \mathrm{H}$ NMR: $\quad\left(500 \mathrm{MHz}, \mathrm{CDCl}_{3}\right)$

7.32-7.66 (m, $5 \mathrm{H}$, Aryl); $4.92(\mathrm{~s}, 1 \mathrm{H}, \mathrm{OH}), 3.77\left(\mathrm{~s}, 3 \mathrm{H}, \mathrm{OCH}_{3}\right) ; 2.92(\mathrm{AB}$ quartet, $J=15.9,2 \mathrm{H}$, $\left.\mathrm{H}_{2} \mathrm{C}(2)\right) ; 2.65$ (s, $\left.1 \mathrm{H}, \mathrm{HC}(5)\right)$

${ }^{13} \mathrm{C}$ NMR: $\quad\left(100 \mathrm{MHz}, \mathrm{CDCl}_{3}\right)$

$172.2(\mathrm{C}(1)), 142.5\left(\mathrm{C}\left(1^{\prime}\right)\right), 129.4\left(\mathrm{C}\left(3^{\prime}\right)\right), 126.8$ (C(4')), $125.1\left(\mathrm{C}\left(2^{\prime}\right)\right), 90.0(\mathrm{C}(4))$, $73.6(\mathrm{C}(3)), 69.7(\mathrm{C}(5)), 52.1(\mathrm{C}(\mathrm{OMe})), 48.4(\mathrm{C}(2))$

IR: (neat)

3476 (s), 3297 (s), 3062 (w), 3032 (w), 2956 (m), 2111(w), 1719 (s), 1602 (w), 1492 (w), 1450 (s), 1439 (s), 1324 (s), 1180 (s), 1061 (s), 943 (m)

TLC: $\quad R_{f} 0.27$ (hexane/EtOAc, 5/1)

SFC: $\quad t_{\mathrm{R}} 5.30 \min (49.9 \%) ; t_{\mathrm{R}} 6.11 \min (50.1 \%)\left(\right.$ Chiracel OJ, $2.5 \% \mathrm{MeOH}$ in $\mathrm{CO}_{2}, 150$ bar, $40{ }^{\circ} \mathrm{C}, 3.0 \mathrm{~mL} \mathrm{~min}^{-1}$ )

MS: (FI, $70 \mathrm{eV})$ 205 (14), 204 (100)

Analysis: $\quad \mathrm{C}_{12} \mathrm{H}_{12} \mathrm{O}_{3}(204.23)$
Calcd:
C, 70.57;
H, $5.92 \%$
Found:
C, 70.73;
H, $5.78 \%$ 
Following General Procedure II, ketone (2c) $(260 \mathrm{mg}, 2.0 \mathrm{mmol})$, trichlorosilyl ketene acetal 1 (380 $\mu \mathrm{L}, 2.4 \mathrm{mmol}, 1.2$ equiv) and bis- $N$-oxide $(P)-(R, R)-19$ (102 mg, $0.2 \mathrm{mmol}, 0.1$ equiv) were stirred at $-20{ }^{\circ} \mathrm{C}$ for $12 \mathrm{~h}$. Work-up provided $533 \mathrm{mg}$ of crude product. Analytically pure product (363 mg, 89\%) was obtained as a colorless liquid after bulb-to-bulb distillation.

Data for $(+)-3 \mathbf{c}:$

SFC: $\quad$ major, $t_{\mathrm{R}} 5.34 \mathrm{~min}(93.1 \%)$; minor, $t_{\mathrm{R}} 6.19 \mathrm{~min}(6.9 \%)($ Chiracel OJ, $2.5 \% \mathrm{MeOH}$ in $\mathrm{CO}_{2}, 150$ bar, $40{ }^{\circ} \mathrm{C}, 3.0 \mathrm{~mL} \mathrm{~min}^{-1}$ )

Opt. Rot.: $[\alpha]_{\mathrm{D}}^{24}+9.3(c=1.53, \mathrm{EtOH})$

Analysis: $\quad \mathrm{C}_{12} \mathrm{H}_{12} \mathrm{O}_{3}(204.23)$
Calcd:
C, 70.57;
$\mathrm{H}, 5.92 \%$
Found:
C, 70.44;
$\mathrm{H}, 5.90 \%$

Methyl (1-Hydroxy-1,2,3,4-tetrahydro-1-naphthalenyl)acetate (( ()$-3 d)$

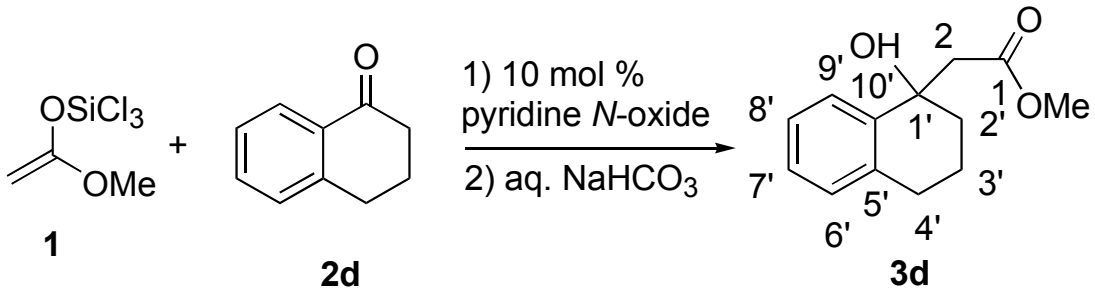

Following General Procedure I, 1-tetralone (2d) $(265 \mu \mathrm{L}, 2.0 \mathrm{mmol})$, trichlorosilyl ketene acetal 1 (380 $\mu \mathrm{L}, 2.4 \mathrm{mmol}, 1.2$ equiv) and pyridine $N$-oxide (19.0 mg, $0.2 \mathrm{mmol}, 0.1$ equiv) were stirred at ambient temperature for $2 \mathrm{~h}$. Work-up provided $434 \mathrm{mg}$ of crude product. Analytically pure product (386 mg, 87\%) was obtained as a colorless liquid after bulb-to-bulb distillation.

Data for $( \pm)-3 d:$

bp: $\quad 110^{\circ} \mathrm{C} \operatorname{ABT}(0.2 \mathrm{mmHg})$

${ }^{1} \mathrm{H}$ NMR: $\quad\left(400 \mathrm{MHz}, \mathrm{CDCl}_{3}\right)$

7.55-7.57 (dd, $\left.J=6.8,1.7,1 \mathrm{H}, \mathrm{HC}\left(9^{\prime}\right)\right)$; 7.16-7.24 (m, $\left.2 \mathrm{H}, \mathrm{HC}\left(7^{\prime}\right) \mathrm{HC}\left(8^{\prime}\right)\right)$; 7.07-

$7.09\left(\mathrm{dd}, J=6.8,0.7,1 \mathrm{H}, \mathrm{HC}\left(6^{\prime}\right)\right) ; 3.92$ (bs, $\left.1 \mathrm{H}, \mathrm{OH}\right) ; 3.73\left(\mathrm{~s}, 3 \mathrm{H}, \mathrm{OCH}_{3}\right) ; 2.84$

(AB quartet, $\left.J=15.6,2 \mathrm{H}, \mathrm{H}_{2} \mathrm{C}(2)\right)$; 2.73-2.84 (m, $2 \mathrm{H}, \mathrm{H}_{2} \mathrm{C}(4)$ )) 1.75-2.13 (m, 4 $\left.\mathrm{H}, \mathrm{H}_{2} \mathrm{C}\left(2^{\prime}\right) \mathrm{H}_{2} \mathrm{C}\left(3^{\prime}\right)\right)$ 
${ }^{13} \mathrm{C}$ NMR: $\quad\left(100 \mathrm{MHz}, \mathrm{CDCl}_{3}\right)$

$172.9(\mathrm{C}(1)), 140.5\left(\mathrm{C}\left(10^{\prime}\right)\right), 136.4\left(\mathrm{C}\left(5^{\prime}\right)\right), 129.8\left(\mathrm{C}\left(8^{\prime}\right)\right), 127.4\left(\mathrm{C}\left(7^{\prime}\right)\right), 126.3$ $\left(\mathrm{C}\left(9^{\prime}\right.\right.$ or $\left.\left.6^{\prime}\right)\right), 126.2\left(\mathrm{C}\left(9^{\prime}\right.\right.$ or $\left.\left.\left.6^{\prime}\right)\right), 71.2(\mathrm{C}(2)), 51.8\left(\mathrm{OCH}_{3}\right)\right), 45.8\left(\mathrm{C}\left(2^{\prime}\right)\right), 36.2$ $\left(\mathrm{C}\left(4^{\prime}\right)\right), 23.4\left(\mathrm{C}\left(2^{\prime}\right)\right), 20.0\left(\mathrm{C}\left(3^{\prime}\right)\right)$

IR: (neat)

3497 (s), 3061 (w), 3021 (w), 2947 (s), 2370 (w), 2340 (w), 1729 (s), 1605 (w), 1489 (m), 1437 (s), 1336 (s), 1278(s), 1203 (s), 1171 (s), 1087 (m), 973 (m), 947 (w)

TLC: $\quad R_{f} 0.39$ (hexane/EtOAc, 5/1)

SFC: $t_{\mathrm{R}} 4.93 \min (50.0 \%) ; t_{\mathrm{R}} 5.68 \mathrm{~min}(50.0 \%)$ (Chiracel OJ, $1.3 \% \mathrm{MeOH}$ in $\mathrm{CO}_{2}, 150$ bar, $40{ }^{\circ} \mathrm{C}, 2.5 \mathrm{~mL} \mathrm{~min}^{-1}$ )

MS: $\quad(\mathrm{EI}, 70 \mathrm{eV})$

202 (35), 147 (100), 129 (65), 115 (13), 103 (5), 91 (35), 77 (5)

Analysis: $\quad \mathrm{C}_{13} \mathrm{H}_{16} \mathrm{O}_{3}(220.27)$

$\begin{array}{lll}\text { Calcd: } & \text { C, 70.88; } & \text { H, 7.32\% } \\ \text { Found: } & \text { C, 70.80; } & \text { H, 7.37\% }\end{array}$

Following General Procedure II, 1-tetralone (2d) (265 $\mu \mathrm{L}, 2.0 \mathrm{mmol})$, trichlorosilyl ketene acetal 1 (380 $\mu \mathrm{L}, 2.4 \mathrm{mmol}, 1.2$ equiv) and bis- $N$-oxide $(P)-(R, R)-19$ (102 mg, $0.2 \mathrm{mmol}$, 0.1 equiv) were stirred at $-20{ }^{\circ} \mathrm{C}$ for $12 \mathrm{~h}$. Work-up provided $492 \mathrm{mg}$ of crude product. Analytically pure product (395 mg, 90\%) was obtained as a colorless liquid after bulb-to-bulb distillation.

Data for (-)-3d:

SFC: $\quad$ minor, $t_{\mathrm{R}} 4.93 \mathrm{~min}(9.9 \%)$; major, $t_{\mathrm{R}} 5.68 \min (90.1 \%)$ (Chiracel OJ, $1.3 \% \mathrm{MeOH}$ in $\mathrm{CO}_{2}, 150$ bar, $40{ }^{\circ} \mathrm{C}, 2.5 \mathrm{~mL} \mathrm{~min}^{-1}$ )

Opt. Rot.: $[\alpha]_{\mathrm{D}}^{24}-19.6(c=1.86, \mathrm{EtOH})$

Analysis: $\quad \mathrm{C}_{13} \mathrm{H}_{16} \mathrm{O}_{3}(220.27)$
Calcd:
C, 70.88;
$\mathrm{H}, 7.32 \%$
Found:
C, 70.70;
H, $7.23 \%$ 
Methyl 3-Hydroxy-3-(1'-naphthalenyl)butanoate (( \pm$)-3 e)$

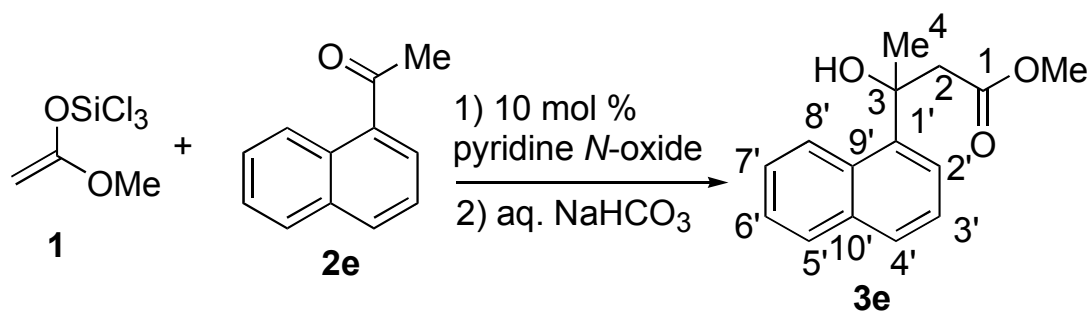

Following General Procedure I, 1-acetonaphthone (2e) $(151 \mu \mathrm{L}, 1.0 \mathrm{mmol})$, trichlorosilyl ketene acetal 1 (190 $\mu \mathrm{L}, 1.2 \mathrm{mmol}, 1.2$ equiv) and pyridine $N$-oxide (9.4 mg, $0.1 \mathrm{mmol}, 0.1$ equiv) were stirred at ambient temperature for $16 \mathrm{~h}$. Work-up provided $245 \mathrm{mg}$ of crude product. Analytically pure product $(217 \mathrm{mg}, 89 \%)$ was obtained as colorless oil after bulb-to-bulb distillation.

Data for $( \pm)-\mathbf{3 e}$ :

bp: $\quad 150{ }^{\circ} \mathrm{C} \operatorname{ABT}(0.1 \mathrm{mmHg})$

${ }^{1} \underline{\mathrm{H} N M R}: \quad\left(400 \mathrm{MHz}, \mathrm{CDCl}_{3}\right)$

8.1(d, $\left.J=8.6,1 \mathrm{H}, \mathrm{HC}\left(2^{\prime}\right)\right) ; 7.85(\mathrm{dd}, J=8.0,0.7,1 \mathrm{H}) ; 7.77(\mathrm{~d}, J=8.3,1 \mathrm{H}$, $\left.\mathrm{HC}\left(4^{\prime}\right)\right)$; 7.45-7.54 (m, 3 H), 7.39 (t, 7.8, 1 H, C(3') ); 4.61 (s, 1 H, OH), 3.67 (s, 3 $\left.\mathrm{H}, \mathrm{OCH}_{3}\right) ; 3.21\left(\mathrm{AB}\right.$ quartet, $\left.J=16.3,2 \mathrm{H}, \mathrm{H}_{2} \mathrm{C}(2)\right) ; 1.86\left(\mathrm{~s}, 3 \mathrm{H}, \mathrm{H}_{3} \mathrm{C}(4)\right.$ )

${ }^{13} \mathrm{C}$ NMR: $\quad\left(100 \mathrm{MHz}, \mathrm{CDCl}_{3}\right)$

$173.5(\mathrm{C}(1)), 141.3,135.0,130.8,129.2,129.9,126.9,125.2,125.2$, 124.7, 122.7, 74.4 (C(3)), 51.8 (OMe), 45.9 (C(2)), 29.8 (C(4))

IR: (neat)

3492 (s), 3096 (w), 3048 (m), 2981 (s), 2952 (s), 2362 (w), 1938 (w), 1716 (s), 1599 (m), 1509 (m), 1437 (s), 1372 (s), 1343 (s), 1204 (s), 1177 (s), 1113 (s), 1081 (s), 1061 (m), 1008 (m), $956(\mathrm{~m}), 871(\mathrm{w})$

TLC: $\quad R_{f} 0.27$ (hexane/EtOAc, 5/1)

SFC: $\quad t_{\mathrm{R}} 4.72 \min (50.1 \%) ; t_{\mathrm{R}} 5.17 \mathrm{~min}(49.9 \%)$ (Chiracel AS, $1.8 \mathrm{~mol} \% \mathrm{MeOH}$ in $\mathrm{CO}_{2}, 150$ bar, $40{ }^{\circ} \mathrm{C}, 2.5 \mathrm{~mL}$ min-1)

MS: $\quad(\mathrm{FI}, 70 \mathrm{eV})$ 245 (15), 244 (100), 170 (4) 
Analysis: $\quad \mathrm{C}_{15} \mathrm{H}_{16} \mathrm{O}_{3}(244.29)$
Calcd:
C, 73.75;
$\mathrm{H}, 6.60 \%$
Found:
C, 73.58;
$\mathrm{H}, 6.24 \%$

Following General Procedure II, 1-acetonaphthone (2e) (302 $\mu \mathrm{L}, 2.0 \mathrm{mmol}$ ), trichlorosilyl ketene acetal $\mathbf{1}(380 \mu \mathrm{L}, 1.2 \mathrm{mmol}, 1.2$ equiv) and bis- $N$-oxide $(P)-(R, R)-\mathbf{1 9}(102$ $\mathrm{mg}, 0.2 \mathrm{mmol}, 0.1$ equiv) were stirred at $-20{ }^{\circ} \mathrm{C}$ for $20 \mathrm{~h}$. Work-up provided $530 \mathrm{mg}$ of crude product. Analytically pure product $(430 \mathrm{mg}, 89 \%)$ was obtained as colorless oil after bulb-tobulb distillation.

Data for $(+)-3 e$ :

SFC: $\quad$ minor, $t_{\mathrm{R}} 4.65 \mathrm{~min}(22.1 \%)$; major, $t_{\mathrm{R}} 5.17 \mathrm{~min}$ (77.9\%) (Chiracel AS, $1.8 \mathrm{~mol} \%$ $\mathrm{MeOH}$ in $\mathrm{CO}_{2}, 150$ bar, $40{ }^{\circ} \mathrm{C}, 2.5 \mathrm{~mL}$ min$^{-1}$ )

Opt. Rot.: $[\alpha]_{\mathrm{D}}^{24}+13.7(c=1.3, \mathrm{EtOH})$

Analysis: $\quad \mathrm{C}_{15} \mathrm{H}_{16} \mathrm{O}_{3}(244.23)$
Calcd:
C, 73.75;
$\mathrm{H}, 6.60 \%$
Found:
C, 73.58;
$\mathrm{H}, 6.24 \%$ 
Methyl 3-Hydroxy-3-(2'-furyl)butanoate (( \pm$)-3 f)$

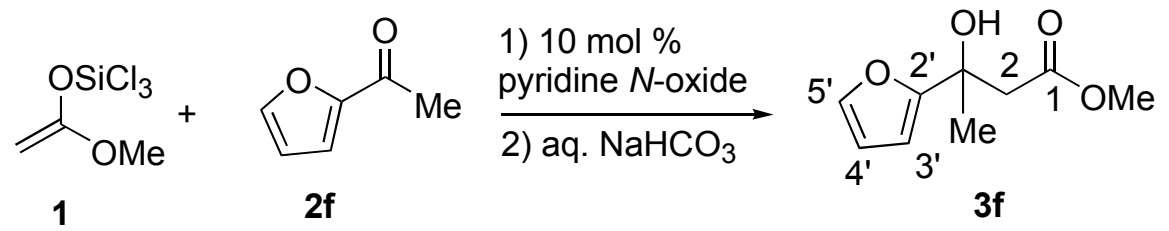

Following General Procedure I, 2-furyl methyl ketone (2f) (200 $\mu \mathrm{L}, 2.0 \mathrm{mmol})$, trichlorosilyl ketene acetal 1 (380 $\mu \mathrm{L} 2.4 \mathrm{mmol}, 1.2$ equiv) and pyridine $N$-oxide (19.0 mg, 0.2 mmol, 0.1 equiv) were stirred at $\mathrm{rt}$ for $2 \mathrm{~h}$. Work-up provided $344 \mathrm{mg}$ of crude product. Analytically pure product $306 \mathrm{mg}(91 \%)$ was obtained as a colorless liquid after bulb-to-bulb distillation.

Data for $( \pm)-\mathbf{3 f}$ :

bp: $\quad 90{ }^{\circ} \mathrm{C}(\mathrm{ABT} 2 \mathrm{mmHg})$

${ }^{1} \mathrm{H}$ NMR: $\quad\left(400 \mathrm{MHz}, \mathrm{CDCl}_{3}\right)$

$7.32\left(\mathrm{dd}, J=1.7,0.7,1 \mathrm{H}, \mathrm{HC}\left(3^{\prime}\right)\right) ; 6.30\left(\mathrm{dd}, J=3.2,1.7,1 \mathrm{H}, \mathrm{HC}\left(4^{\prime}\right)\right) ; 6.24(\mathrm{dd}$, $\left.J=3.2,0.8,1 \mathrm{H}, \mathrm{HC}\left(5^{\prime}\right)\right) ; 4.30(\mathrm{bs}, 1 \mathrm{H}, \mathrm{OH}) ; 3.68\left(\mathrm{~s}, 3 \mathrm{H}, \mathrm{OCH}_{3}\right) ; 2.89(\mathrm{AB}$ quartet, $\left.J=16.0,2 \mathrm{H}, \mathrm{H}_{2} \mathrm{C}(2)\right) ; 1.58\left(\mathrm{~s}, 3 \mathrm{H}, \mathrm{H}_{3} \mathrm{C}(4)\right)$

${ }^{13} \mathrm{C} \mathrm{NMR:} \quad\left(100 \mathrm{MHz}, \mathrm{CDCl}_{3}\right)$

$172.7(\mathrm{C}(1)), 158.3\left(\mathrm{C}\left(1^{\prime}\right)\right), 141.5\left(\mathrm{C}\left(4^{\prime}\right)\right), 110.1\left(\mathrm{C}\left(3^{\prime}\right)\right), 104.5\left(\mathrm{C}\left(2^{\prime}\right)\right), 69.5(\mathrm{C}(3))$, $51.8\left(\mathrm{OCH}_{3}\right), 44.1(\mathrm{C}(2)), 27.5(\mathrm{C}(4))$

IR: (neat)

3485 (s), 2987 (s), 2953 (s), 1731 (s), 1490 (s), 1370 (s), 1296 (m), 1205 (m), $1117(\mathrm{~m}), 944(\mathrm{w})$

TLC: $\quad R f 0.33$ (hexane/EtOAc, 5/1)

SFC: $\quad t_{\mathrm{R}} 2.99 \min (49.8 \%) ; t_{\mathrm{R}} 3.44 \min (50.2 \%)\left(\right.$ Chiracel $\mathrm{AD}, 1.5 \% \mathrm{MeOH}$ in $\mathrm{CO}_{2}$, 150 bar, $40{ }^{\circ} \mathrm{C}, 2.5 \mathrm{~mL} \min ^{-1}$ )

MS: $\quad(\mathrm{EI}, 70 \mathrm{eV})$

184 (15), 169 (15), 153 (5), 137 (7), 111 (100), 95 (35), 79 (7), 59 (7)

Analysis: $\quad \mathrm{C}_{9} \mathrm{H}_{12} \mathrm{O}_{3}$ (184.19)
Calcd:
C, 58.69;
H, $6.57 \%$
Found:
C, 58.55;
$\mathrm{H}, 6.58 \%$ 
Following General Procedure II, 2-furyl methyl ketone (2f) (200 $\mu \mathrm{L}, 2.0 \mathrm{mmol})$, trichlorosilyl ketene acetal 1 (380 $\mu \mathrm{L} 2.4 \mathrm{mmol}, 1.2$ equiv) and bis- $N$-oxide $(P)-(R, R)-19(102$ $\mathrm{mg}, 0.2 \mathrm{mmol}, 0.1$ equiv) were stirred at $-20{ }^{\circ} \mathrm{C}$ for $12 \mathrm{~h}$. Work-up provided $440 \mathrm{mg}$ of crude product. Analytically pure product $(298 \mathrm{mg}, 87 \%)$ was obtained as a colorless liquid after bulbto-bulb distillation.

Data for (-)-3f:

SFC: $\operatorname{minor}, t_{\mathrm{R}} 3.03$ min $(25.7 \%)$; major, $t_{\mathrm{R}} 3.47 \min (74.3 \%)$ (Chiracel AD, $1.5 \%$ $\mathrm{MeOH}$ in $\mathrm{CO}_{2}, 150$ bar, $40{ }^{\circ} \mathrm{C}, 2.5 \mathrm{~mL} \mathrm{~min}^{-1}$ )

Opt. Rot.: $[\alpha]_{\mathrm{D}}^{24}-3.11(c=2.7, \mathrm{EtOH})$

Analysis: $\quad \mathrm{C}_{9} \mathrm{H}_{12} \mathrm{O}_{3}(184.19)$
Calcd:
C, 58.69;
$\mathrm{H}, 6.57 \%$
Found:
C, 58.55;
$\mathrm{H}, 6.58 \%$

\section{Methyl 3-Hydroxy-3-methyl-5-phenyl-4-pentynoate ((土)-3g)}

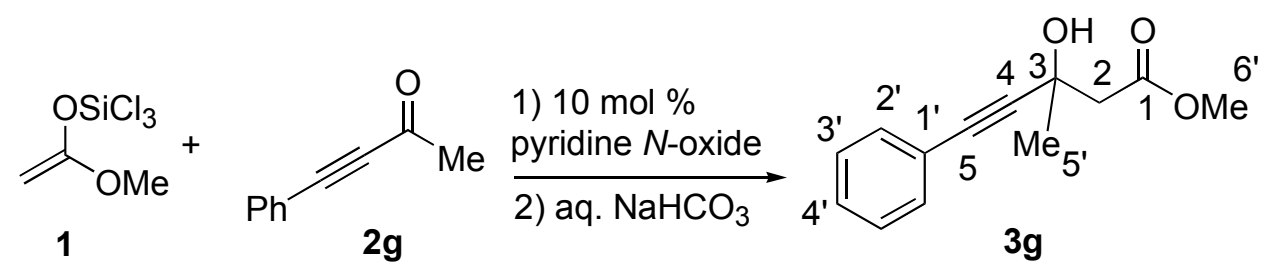

Following General Procedure I, 4-phenyl-3-butyn-2-one (2g) (290 $\mu \mathrm{L}, 2.0 \mathrm{mmol})$, trichlorosilyl ketene acetal $1(380 \mu \mathrm{L}, 2.4 \mathrm{mmol}, 1.2$ equiv) and pyridine $N$-oxide (19.0 mg, 0.2 mmol, 0.1 equiv) were stirred at ambient temperature for $2 \mathrm{~h}$. Work-up provided $244 \mathrm{mg}$ of crude product. Analytically pure product (402 mg, 92\%) was obtained as a colorless liquid after bulb-to-bulb distillation.

\section{Data for $( \pm)-3 g:$}

bp: $\quad 102^{\circ} \mathrm{C}(\mathrm{ABT} 0.2 \mathrm{mmHg})$

${ }^{1}$ H NMR: $\quad\left(400 \mathrm{MHz}, \mathrm{CDCl}_{3}\right)$

7.29-7.41 (m, $5 \mathrm{H}$, Aryl); $4.32(\mathrm{~s}, 1 \mathrm{H}, \mathrm{OH}) ; 3.77\left(\mathrm{~s}, 3 \mathrm{H}, \mathrm{OCH}_{3}\right) ; 2.80(\mathrm{AB}$ quartet, $\left.J=15.9,2 \mathrm{H}, \mathrm{H}_{2} \mathrm{C}(2)\right) ; 1.63\left(\mathrm{~s}, 3 \mathrm{H}, \mathrm{H}_{3} \mathrm{C}\left(5^{\prime}\right)\right)$ 
${ }^{13}$ C NMR: $\quad\left(100 \mathrm{MHz}, \mathrm{CDCl}_{3}\right)$

$172.4(\mathrm{C}(1)), 131.7\left(\mathrm{C}\left(2^{\prime}\right)\right), 129.4\left(\mathrm{C}\left(4^{\prime}\right)\right), 129.2\left(\mathrm{C}\left(3^{\prime}\right)\right), 122.4\left(\mathrm{C}\left(1^{\prime}\right)\right), 91.2(\mathrm{C}(5))$, $83.0(\mathrm{C}(4)), 65.6(\mathrm{C}(3)), 51.9\left(\mathrm{C}\left(6^{\prime}\right)\right), 24.8(\mathrm{C}(2)), 23.5\left(\mathrm{C}\left(5^{\prime}\right)\right)$

IR: (neat) $3487(\mathrm{~s}), 3120(\mathrm{w}), 2986$ (s), 2954 (s), 1732 (s), 1624 (w), 1505 (w), 1439 (s), 1347 (s), 1294 (s), 1180 (s), 1095 (s), 1010 (s), 939 (m)

TLC: $\quad R_{f} 0.40$ (hexane/EtOAc, 5/1)

SFC: $\quad t_{\mathrm{R}} 4.06 \min (49.5 \%) ; t_{\mathrm{R}} 4.43 \min (50.5 \%)$ (Chiracel OJ, $1.0 \% \mathrm{MeOH}$ in $\mathrm{CO}_{2}, 150$ bar, $40{ }^{\circ} \mathrm{C}, 2.5 \mathrm{~mL} \mathrm{~min}^{-1}$ )

MS: $\quad(\mathrm{EI}, 70 \mathrm{eV})$ 218 (17), 203 (10), 161 (7), 145 (100), 129 (40), 101 (15), 77 (7)

Analysis: $\quad \mathrm{C}_{13} \mathrm{H}_{14} \mathrm{O}_{3}(218.15)$
Calcd:
C, 71.54;
$\mathrm{H}, 6.47 \%$

Found:

C, 71.42;

$\mathrm{H}, 6.42 \%$

Following General Procedure II, 4-phenyl-3-butyn-2-one (2g) (290 $\mu \mathrm{L}, 2.0 \mathrm{mmol})$, trichlorosilyl ketene acetal 1 (380 $\mu \mathrm{L}, 2.4 \mathrm{mmol}, 1.2$ equiv) and bis- $N$-oxide $(P)-(R, R)-19$ (102 mg, $0.2 \mathrm{mmol}$, 0.1 equiv) were stirred at $-20{ }^{\circ} \mathrm{C}$ for $20 \mathrm{~h}$. Work-up provided $244 \mathrm{mg}$ of crude product. Analytically pure product (402 mg, 92\%) was obtained as a colorless liquid after bulb-to-bulb distillation.

\section{Data for (-)-3g:}

SFC: $\operatorname{minor}, t_{\mathrm{R}} 4.16 \min (32.4 \%)$; major, $t_{\mathrm{R}} 4.54 \min (67.6 \%)$ (Chiracel OJ, $1.0 \%$, $\mathrm{MeOH}$ in $\mathrm{CO}_{2}, 150$ bar, $40{ }^{\circ} \mathrm{C}, 2.5 \mathrm{~mL} \mathrm{~min}^{-1}$ )

Opt. Rot.: $[\alpha]_{\mathrm{D}}^{24}-2.6(c=3.52, \mathrm{EtOH})$

Analysis: $\quad \mathrm{C}_{13} \mathrm{H}_{14} \mathrm{O}_{3}(218.15)$
Calcd:
C, 71.54;
$\mathrm{H}, 6.47 \%$
Found:
C, 71.26;
$\mathrm{H}, 6.50 \%$ 
Methyl 3-Hydroxy-3-(4'-trifluoromethyl phenyl)butanoate (( \pm$)-3 h)$

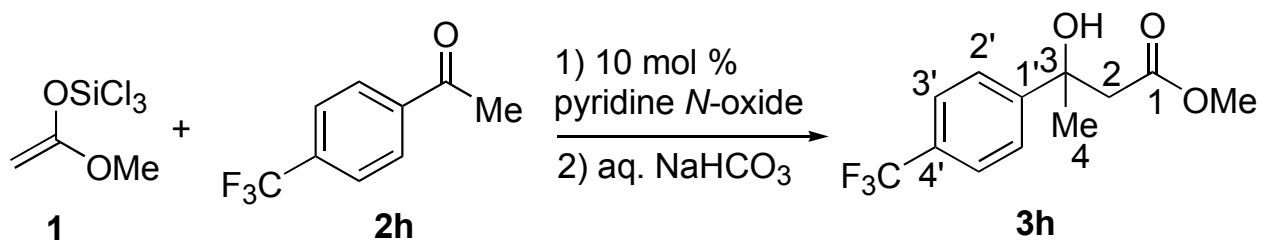

Following General Procedure I, 4'-trifluoromethyl acetophenone (2h) (188 mg, 1.0 mmol), trichlorosilyl ketene acetal 1 (190 $\mu \mathrm{L}, 1.2 \mathrm{mmol}, 1.2$ equiv) and pyridine $N$-oxide (9.4 $\mathrm{mg}, 0.1 \mathrm{mmol}, 0.1$ equiv) were stirred at $\mathrm{rt}$ for $4 \mathrm{~h}$. Work-up provided $271 \mathrm{mg}$ of crude product. Analytically pure product $(250 \mathrm{mg}, 90 \%)$ was obtained as colorless oil after bulb-to-bulb distillation.

Data for $( \pm)-3 h$ :

bp: $\quad 120{ }^{\circ} \mathrm{C} \mathrm{ABT}(0.1 \mathrm{mmHg})$

${ }^{1}$ H NMR: $\quad\left(400 \mathrm{MHz}, \mathrm{CDCl}_{3}\right)$

7.59 (AB quartet, $J=8.6,4 \mathrm{H}, \mathrm{HC}\left(2^{\prime}\right)$ and $\left.\mathrm{HC}\left(3^{\prime}\right)\right) ; 4.45(\mathrm{~s}, 1 \mathrm{H}, \mathrm{OH}) ; 3.62$ (s, 3

$\left.\mathrm{H}, \mathrm{OCH}_{3}\right) ; 2.92\left(\mathrm{AB}\right.$ quartet, $\left.J=16.1,2 \mathrm{H}, \mathrm{H}_{2} \mathrm{C}(2)\right) ; 1.54$ (s, $3 \mathrm{H}, \mathrm{H}_{3} \mathrm{C}(4)$ )

${ }^{13} \mathrm{C}$ NMR: $\quad\left(100 \mathrm{MHz}, \mathrm{CDCl}_{3}\right)$

$173.0(\mathrm{C}(1)), 150.9\left(\mathrm{C}\left(1^{\prime}\right)\right), 129.2$ (quartet, $J=32.2, \mathrm{C}\left(4^{\prime}\right)$ ), 125.3 (quartet, $J=$

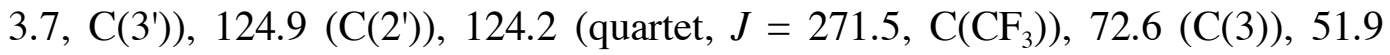

(C(OMe)), 45.7 (C(2)), 30.5 (C(4))

${ }^{19}$ F NMR: $\quad(376 \mathrm{MHz})$

$-62.86$

IR: (neat)

3500 (s), 2983 (m), 2958 (m), 2648 (w), 1923 (w), 1720 (s), 1620 (s), 1583 (w),

1411 (s), 1440 (s), 1372 (s), 1278 (s), 1211 (s), 1166 (s), 1124 (s), 1089 (s), 1075

(s), $1015(\mathrm{~s}), 961(\mathrm{~m}), 824(\mathrm{~s})$

TLC: $\quad R_{f} 0.24$ (hexane/EtOAc, 5/1)

SFC: $t_{\mathrm{R}} 1.59 \min (50.5 \%) ; t_{\mathrm{R}} 1.77 \mathrm{~min}(49.5 \%)$ (Chiracel $\mathrm{AD}, 3.0 \mathrm{~mol} \% \mathrm{MeOH}$ in $\mathrm{CO}_{2}, 150$ bar, $40{ }^{\circ} \mathrm{C}, 3.0 \mathrm{~mL} \mathrm{~min}^{-1}$ ) 
MS: $\quad(F I, 70 \mathrm{eV})$

262 (21), 248 (12), 247 (100)

Analysis: $\quad \mathrm{C}_{12} \mathrm{H}_{13} \mathrm{~F}_{3} \mathrm{O}_{3}(262.23)$
Calcd:
C, 54.96;
$\mathrm{H}, 5.00 \%$
Found:
C, 55.11;
$\mathrm{H}, 5.30 \%$

Following General Procedure II, 4'-trifluoromethyl acetophenone (2h) $(380 \mathrm{mg}, 2.0$ mmol), trichlorosilyl ketene acetal 1 ( $380 \mu \mathrm{L}, 2.4 \mathrm{mmol}, 1.2$ equiv) and bis- $N$-oxide $(P)-(R, R)-19$ (102 mg, $0.2 \mathrm{mmol}, 0.1$ equiv) were stirred at $-20{ }^{\circ} \mathrm{C}$ for $20 \mathrm{~h}$. Work-up provided $590 \mathrm{mg}$ of crude product. Analytically pure product (504 mg, 91\%) was obtained as colorless oil after bulbto-bulb distillation.

Data for $(-)-3 \mathbf{h}$ :

SFC: $\quad$ minor, $t_{\mathrm{R}} 1.62 \mathrm{~min}(12.0 \%)$; major, $t_{\mathrm{R}} 1.80 \mathrm{~min}(88.0 \%)($ Chiracel AD, $3.0 \mathrm{~mol} \%$ $\mathrm{MeOH}$ in $\mathrm{CO}_{2}, 150 \mathrm{bar}, 40{ }^{\circ} \mathrm{C}, 3.0 \mathrm{~mL} \mathrm{~min}^{-1}$ )

Opt. Rot.: $\quad[\alpha]_{\mathrm{D}}^{24}-5.9(c=4.68, \mathrm{EtOH})$

Analysis: $\quad \mathrm{C}_{12} \mathrm{H}_{13} \mathrm{~F}_{3} \mathrm{O}_{3}(262.23)$

$\begin{array}{lll}\text { Calcd: } & \text { C, 54.96; } & \text { H, } 5.00 \% \\ \text { Found: } & \text { C, 55.11; } & \text { H, } 5.30 \%\end{array}$

\section{Methyl 3-Hydroxy-3-(4'-methoxy phenyl)butanoate (( ()$-3 i)$}

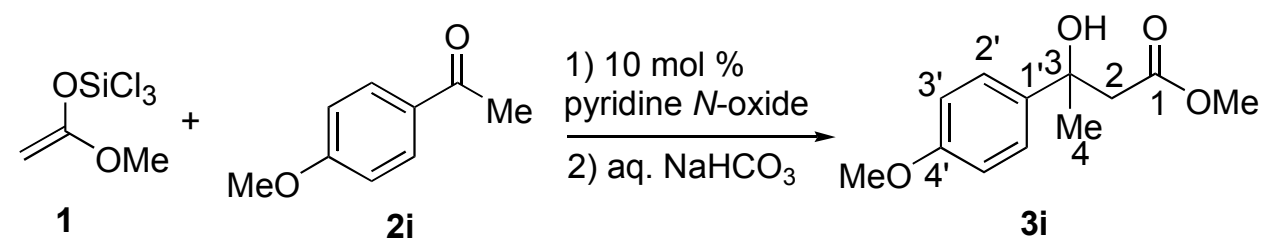

Following General Procedure I, 4'-methoxy acetophenone (2i) $(150 \mathrm{mg}, 1.0 \mathrm{mmol})$, trichlorosilyl ketene acetal 1 (190 $\mu \mathrm{L}, 1.2 \mathrm{mmol}, 1.2$ equiv) and pyridine $N$-oxide (9.4 mg, 0.1 mmol, 0.1 equiv) were stirred at ambient temperature for $2 \mathrm{~h}$. Work-up provided $226 \mathrm{mg}$ of crude product. Analytically pure product (208 mg, 94\%) was obtained as a colorless liquid after bulb-to-bulb distillation. 


\section{Data for $( \pm)-3 \mathbf{i}:$}

bp: $\quad 140^{\circ} \mathrm{C} \operatorname{ABT}(0.5 \mathrm{mmHg})$

11 H NMR: $\quad\left(400 \mathrm{MHz}, \mathrm{CDCl}_{3}\right)$

7.35-7.37 (m, 2 H, H(2')), 6.85-6.88 (m, H(3')); 4.27 (s, $1 \mathrm{H}, \mathrm{OH}) ; 3.80$ (s, $3 \mathrm{H}$, $\mathrm{OCH}_{3}$ ); 2.87 (AB quartet, $\mathrm{J}=16.0,2 \mathrm{H}, \mathrm{H}_{2} \mathrm{C}(2)$ ); 1.53 (s, $3 \mathrm{H}, \mathrm{H}_{3} \mathrm{C}(4)$ )

${ }^{13} \mathrm{C}$ NMR: $\quad\left(100 \mathrm{MHz}, \mathrm{CDCl}_{3}\right)$

$173.2(\mathrm{C}(1)), 158.4\left(\mathrm{C}\left(1^{\prime}\right)\right), 139.0\left(\mathrm{C}\left(4^{\prime}\right)\right), 125.6\left(\mathrm{C}\left(3^{\prime}\right)\right), 113.5\left(\mathrm{C}\left(2^{\prime}\right)\right), 72.4(\mathrm{C}(3))$, $55.2(\mathrm{MeOOC}(1)), 51.7\left(\mathrm{MeO} \mathrm{C}\left(4^{\prime}\right)\right), 24.2(\mathrm{C}(2)), 30.7$ (C(4))

IR: (neat)

3504 (s), 2975 (s), 2955 (s), 2937 (m), 2051 (w), 1892 (w), 1718 (s), 1612 (s), 1584 (m), 1513 (s), 1415 (s), 1439 (s), 1371 (s), 1342 (s), 1299 (s), 1208 (s), 1179 (s), $1113(\mathrm{~m}), 1090$ (s), 1010 (m), 833 (s)

TLC: $\quad \operatorname{Rf} 0.19$ (hexane/EtOAc, 5/1)

SFC: $\quad t_{\mathrm{R}} 2.59 \mathrm{~min}(49.9 \%) ; t_{\mathrm{R}} 3.07 \mathrm{~min}(50.1 \%)$ (Chiracel AD, $5 \mathrm{~mol} \% \mathrm{MeOH}$ in $\mathrm{CO} 2$, 150 bar, $40{ }^{\circ} \mathrm{C}, 3.0 \mathrm{~mL}$ min-1)

MS: $\quad(\mathrm{FI}, 70 \mathrm{eV})$ 225 (14), 224 (100)

Analysis: $\quad \mathrm{C}_{12} \mathrm{H}_{16} \mathrm{O}_{4}(224.26)$
Calcd:
C, 64.27;
H, $7.19 \%$
Found:
C, 64.15;
$\mathrm{H}, 7.29 \%$

Following General Procedure II, 4-methoxyacetophenone (2i) (300 mg, 2.0 mmol), trichlorosilyl ketene acetal $1(380 \mu \mathrm{L}, 2.4 \mathrm{mmol}, 1.2$ equiv) and bis- $N$-oxide $(P)-(R, R)$-19 (102 $\mathrm{mg}, 0.2 \mathrm{mmol}, 0.1$ equiv) were stirred at $-20{ }^{\circ} \mathrm{C}$ for $12 \mathrm{~h}$. Work-up provided $556 \mathrm{mg}$ of crude product. Analytically pure product $(420 \mathrm{mg}, 94 \%)$ was obtained as a colorless liquid after bulbto-bulb distillation.

Data for (-)-3i:

SFC: $\quad \operatorname{minor}, t_{\mathrm{R}} 2.59 \min (16.1 \%)$; major, $t_{\mathrm{R}} 3.07 \min (83.9 \%)$ (Chiracel AD, $5 \mathrm{~mol} \%$ $\mathrm{MeOH}$ in $\mathrm{CO}_{2}, 150$ bar, $40{ }^{\circ} \mathrm{C}, 3.0 \mathrm{~mL} \mathrm{~min}^{-1}$ )

Opt. Rot.: $\quad[\alpha]_{D}^{24}-6.4(c=4.44, \mathrm{EtOH})$ 
Analysis: $\quad \mathrm{C}_{12} \mathrm{H}_{16} \mathrm{O}_{4}(224.26)$
Calcd:
C, 64.27;
H, $7.19 \%$
Found:
C, 64.22;
$\mathrm{H}, 7.31 \%$

\section{Methyl (E)-3-Hydroxy-3-methyl-5-phenyl-4-pentenoate (( $( \pm)-3 \mathbf{j})$}

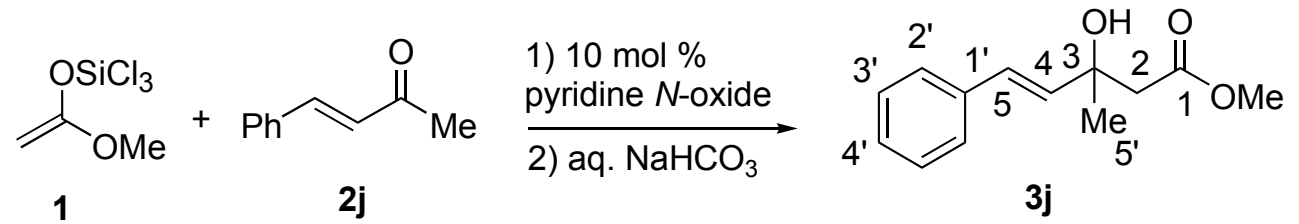

Following General Procedure I, methyl cinnamyl ketone (2j) (292 mg, 2.0 mmol), trichlorosilyl ketene acetal $1(380 \mu \mathrm{L}, 2.4 \mathrm{mmol}, 1.2$ equiv) and pyridine $N$-oxide (19.0 mg, 0.2 mmol, 0.1 equiv) were stirred at $\mathrm{rt}$ for $2 \mathrm{~h}$. Work-up provided $426 \mathrm{mg}$ of crude product. Analytically pure product (410 mg, 92\%) was obtained as a colorless liquid after bulb-to-bulb distillation.

Data for $( \pm)-\mathbf{3 j}$ :

bp: $\quad 110^{\circ} \mathrm{C}$ ABT $(0.2 \mathrm{mmHg})$

${ }^{1}$ H NMR: $\quad\left(400 \mathrm{MHz}, \mathrm{CDCl}_{3}\right)$

7.29-7.39 (m, 5 H, Aryl); 6.65 (d, $J=15.9,1 \mathrm{H}, \mathrm{HC}(5)) ; 6.27$ (d, $J=16.2,1 \mathrm{H}$, $\mathrm{HC}(4)$ ); 3.99 (bs, $1 \mathrm{H}, \mathrm{OH}) ; 3.69$ (s, $3 \mathrm{H}, \mathrm{OCH}_{3}$ ); 2.67 (AB quartet, $J=15.7,2 \mathrm{H}$, $\left.\mathrm{H}_{2} \mathrm{C}(2)\right) ; 1.43$ (s, $\left.3 \mathrm{H}, \mathrm{H}_{3} \mathrm{C}\left(5^{\prime}\right)\right)$

${ }^{13} \mathrm{C}$ NMR: $\quad\left(100 \mathrm{MHz}, \mathrm{CDCl}_{3}\right)$

172.9 (C(1)), $136.7\left(\mathrm{C}\left(1^{\prime}\right)\right), 134.7$ (C(4)), 129.5 (C(5)), 127.9 (C(3')), 127.5 $\left.\left(\mathrm{C}\left(4^{\prime}\right)\right), 126.5\left(\mathrm{C}\left(2^{\prime}\right)\right), 71.2(\mathrm{C}(3)), 51.8\left(\mathrm{OCH}_{3}\right)\right), 45.4(\mathrm{C}(2)), 29.4\left(\mathrm{C}\left(5^{\prime}\right)\right)$

IR: (neat)

3497 (s), 3082 (w), 3059 (w), 3026 (m), 2977 (s), 2953 (s), 1731 (s), 1600 (w), 1578 (w), 1494 (s), 1438 (s), 1370 (s), 1340 (s), 1270 (s), 1206 (s), 1130 (s), 969 (s), $942(\mathrm{~m})$

TLC: $\quad R f 0.31$ (hexane/EtOAc, 5/1) 
SFC: $\quad t_{\mathrm{R}} 6.96 \min (49.9 \%) ; t_{\mathrm{R}} 7.43 \mathrm{~min}(50.1 \%)$ (Chiracel OJ, 1.5\% $\mathrm{MeOH}$ in $\mathrm{CO}_{2}, 150$ bar, $40{ }^{\circ} \mathrm{C}, 2.5 \mathrm{~mL} \mathrm{~min}^{-1}$ )

MS: (EI, $70 \mathrm{eV})$

220 (15), 205 (8), 147 (100), 131 (30), 117 (10), 103 (20), 91 (14), 77 (15)

Analysis: $\quad \mathrm{C}_{13} \mathrm{H}_{16} \mathrm{O}_{3}(220.27)$
Calcd:
C, 70.89;
$\mathrm{H}, 7.32 \%$
Found:
C, 70.60;
$\mathrm{H}, 7.17 \%$

Following General Procedure II, methyl cinnamyl ketone (2j) (292 mg, $2.0 \mathrm{mmol})$, trichlorosilyl ketene acetal 1 (380 $\mu \mathrm{L}, 2.4 \mathrm{mmol}, 1.2$ equiv) and bis- $N$-oxide $(P)-(R, R)-\mathbf{1 9}(102$ $\mathrm{mg}, 0.2 \mathrm{mmol}, 0.1$ equiv) were stirred at $-20{ }^{\circ} \mathrm{C}$ for $12 \mathrm{~h}$. Work-up provided $426 \mathrm{mg}$ of crude product. Analytically pure product $(390 \mathrm{mg}, 87 \%$ ) was obtained as a colorless liquid after bulbto-bulb distillation.

Data for $\mathbf{3 j}$ :

SFC: $\operatorname{minor}, t_{\mathrm{R}} 6.96 \min (44.6 \%)$; major, $t_{\mathrm{R}} 7.43 \mathrm{~min}(55.4 \%)$ (Chiracel OJ, 1.5\% $\mathrm{MeOH}$ in $\mathrm{CO}_{2}, 150 \mathrm{bar}, 40^{\circ} \mathrm{C}, 2.5 \mathrm{~mL} \mathrm{~min}^{-1}$ )

Analysis: $\quad \mathrm{C}_{13} \mathrm{H}_{16} \mathrm{O}_{3}(220.27)$
Calcd:
C, 70.89;
H, $7.32 \%$
Found:
C, 70.74;
H, 7.39\% 
Methyl 1-(1-Hydroxy-2-cyclohexenyl)acetate (( \pm$)-3 k)$

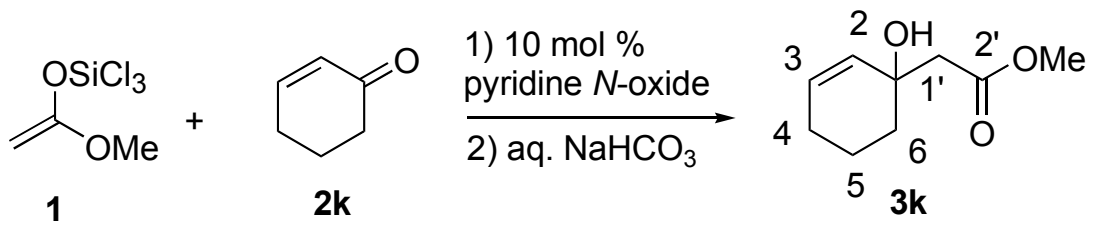

Following General Procedure I, cyclohexenone (2k) (97 $\mu \mathrm{L}, 1.0 \mathrm{mmol})$, trichlorosilyl ketene acetal 1 (190 $\mu \mathrm{L}, 1.2 \mathrm{mmol}, 1.2$ equiv) and pyridine $N$-oxide (10.0 mg, $0.1 \mathrm{mmol}, 0.1$ equiv) were stirred at ambient temperature for $2 \mathrm{~h}$. Work-up provided $182 \mathrm{mg}$ of crude product which was purified by silica gel column chromatography (hexane/EtOAc, 5/1). Analytically pure product (122 mg, 71\%) was obtained as a colorless liquid after bulb-to-bulb distillation.

Data for $( \pm)-3 \mathbf{k}$ :

bp: $\quad 110^{\circ} \mathrm{C} \operatorname{ABT}(0.2 \mathrm{mmHg})$

${ }^{1} \mathrm{H}$ NMR: $\quad\left(400 \mathrm{MHz}, \mathrm{CDCl}_{3}\right)$

5.79-5.83 (m, $1 \mathrm{H}, \mathrm{HC}(3)) ; 5.64(\mathrm{~d}, J=10.0,1 \mathrm{H}, \mathrm{HC}(2)) ; 3.70\left(\mathrm{~s}, 3 \mathrm{H}, \mathrm{OCH}_{3}\right)$;

3.47 (s, $1 \mathrm{H}, \mathrm{OH}) ; 2.55$ (AB quartet, $\left.J=15.7,2 \mathrm{H}, \mathrm{H}_{2} \mathrm{C}(2)\right)$; 1.37-2.07 (m, $6 \mathrm{H}$, $\left.\mathrm{H}_{2} \mathrm{C}(4) \mathrm{H}_{2} \mathrm{C}(5) \mathrm{H}_{2}(6)\right)$

${ }^{13} \mathrm{C}$ NMR: $\quad\left(100 \mathrm{MHz}, \mathrm{CDCl}_{3}\right)$

$173.0\left(\mathrm{C}\left(2^{\prime}\right)\right), 130.8(\mathrm{C}(2)), 130.5(\mathrm{C}(3)), 68.2(\mathrm{C}(1)), 51.7\left(\mathrm{OCH}_{3}\right), 45.2\left(\mathrm{C}\left(1^{\prime}\right)\right)$, $35.7(\mathrm{C}(4)), 25.0(\mathrm{C}(6)), 18.9(\mathrm{C}(5))$

IR: (neat)

3492 (s), 3029 (w), 2939 (s), 2971 (s), 2936 (m), 1732 (s), 1649 (w), 1438 (s), 1407 (m), 1375 (s), 1327 (s), 1178 (s), 1070 (s), 1009 (s), 985 (s), 851 (m)

TLC: $\quad R_{f} 0.29$ (hexane/EtOAc, 5/1)

GC: $\quad t_{\mathrm{R}} 14.00 \mathrm{~min}(49.9 \%) ; t_{\mathrm{R}} 14.41 \mathrm{~min}(50.1 \%)$ (Chiraldex GTA, $\left.90{ }^{\circ} \mathrm{C}, 16 \mathrm{psi}\right)$

MS: $\quad(\mathrm{EI}, 70 \mathrm{eV})$ 170 (2), 153 (12), 142 (22), 121 (10), 110 (15), 97 (100), 85 (4), 79 (45), 68 (50), $55(33)$

Analysis: $\quad \mathrm{C}_{9} \mathrm{H}_{14} \mathrm{O}_{3}(170.21)$

Calcd: $\quad \mathrm{C}, 63.51 ; \quad \mathrm{H}, 8.23 \%$

Found: $\quad$ C, 63.29; $\quad$ H, $8.31 \%$ 
Following General Procedure II, cyclohexenone (2k) (195 $\mu \mathrm{L}, 2.0 \mathrm{mmol})$, trichlorosilyl ketene acetal 1 (380 $\mu \mathrm{L}, 2.4 \mathrm{mmol}, 1.2$ equiv) and bis- $N$-oxide $(P)-(R, R)-19$ (102 mg, $0.2 \mathrm{mmol}$, 0.1 equiv) were stirred at $-20{ }^{\circ} \mathrm{C}$ for $12 \mathrm{~h}$. Work-up provided $480 \mathrm{mg}$ of crude product which was purified by silica gel column chromatography (hexane/EtOAc, 5/1). Analytically pure product (290 mg, 86\%) was obtained as colorless liquid after bulb-to-bulb distillation.

Data for 3k:

GC: $\quad \operatorname{minor}, t_{\mathrm{R}} 14.09 \min (46.1 . \%)$;major, $t_{\mathrm{R}} 14.51 \min (53.9 \%)$ (Chiraldex GTA, 90 ${ }^{\circ} \mathrm{C}, 16$ psi)

Analysis: $\quad \mathrm{C}_{9} \mathrm{H}_{14} \mathrm{O}_{3}(170.21)$

Calcd: $\quad$ C, 63.51; $\quad \mathrm{H}, 8.23 \%$

Found: $\quad$ C, $63.29 ; \quad \mathrm{H}, 8.53 \%$

\section{Methyl 3-Hydroxy-3-methylpentanoate (( \pm$)-31)$}

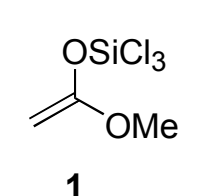

1

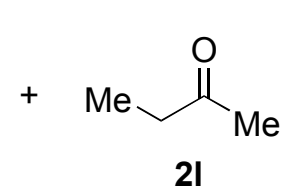

1) $10 \mathrm{~mol} \%$ pyridine $N$-oxide

2) aq. $\mathrm{NaHCO}_{3}$

2l

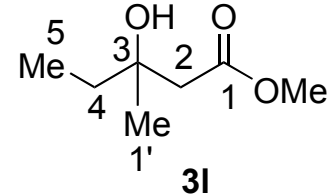

31

Following General Procedure I, 2-butanone (2l) (180 $\mu \mathrm{L}, 2.0 \mathrm{mmol})$, trichlorosilyl ketene acetal 1 (380 $\mu \mathrm{L}, 2.4 \mathrm{mmol}, 1.2$ equiv) and pyridine $N$-oxide (19.0 mg, $0.2 \mathrm{mmol}, 0.1$ equiv) were stirred at ambient temperature for $8 \mathrm{~h}$. Work-up provided $292 \mathrm{mg}$ of crude product. Analytically pure product $(243 \mathrm{mg}, 83 \%$ ) was obtained as a colorless liquid after bulb-to-bulb distillation.

Data for $( \pm)-3 \mathbf{l}$ :

bp: $\quad 100^{\circ} \mathrm{C} \mathrm{ABT}(7 \mathrm{mmHg})$

${ }^{1} \mathrm{H}$ NMR: $\quad\left(400 \mathrm{MHz}, \mathrm{CDCl}_{3}\right)$

$3.71\left(\mathrm{~s}, 3 \mathrm{H}, \mathrm{OCH}_{3}\right) ; 3.52(\mathrm{bs}, 1 \mathrm{H}, \mathrm{OH}) ; 2.50(\mathrm{AB}$ quartet, $J=16.6,2 \mathrm{H}$, $\left.\mathrm{H}_{2} \mathrm{C}(2)\right) ; 1.50-1.59\left(\mathrm{~m}, 2 \mathrm{H}, \mathrm{H}_{2} \mathrm{C}(4)\right) ; 1.21$ (s, $\left.3 \mathrm{H}, \mathrm{H}_{3} \mathrm{C}\left(1^{\prime}\right)\right)$; 0.91 (t, J = 7.6, $3 \mathrm{H}$, $\left.\mathrm{H}_{3} \mathrm{C}(5)\right)$ 
${ }^{13} \mathrm{C} \mathrm{NMR}: \quad\left(100 \mathrm{MHz}, \mathrm{CDCl}_{3}\right)$

$173.7(\mathrm{C}(1)), 71.4(\mathrm{C}(3)), 51.9\left(\mathrm{OCH}_{3}\right), 44.4(\mathrm{C}(2)), 34.8(\mathrm{C}(4)), 26.3\left(\mathrm{C}\left(1^{\prime}\right)\right), 8.5$ $(\mathrm{C}(5))$

IR: (neat)

3509 (s), 2973 (s), 2384 (m), 1735 (s), 1241 (s), 1439 (s), 1209 (s), 1002 (s), 923 (m)

TLC: $\quad R_{f} 0.33$ (hexane/EtOAc, 5/1)

GC: $\quad t_{\mathrm{R}} 7.42 \mathrm{~min}(49.9 \%) ; t_{\mathrm{R}} 7.80 \mathrm{~min}(50.1 \%)$ (Chiraldex GTA, $\left.64{ }^{\circ} \mathrm{C}, 20 \mathrm{psi}\right)$

MS: $\quad(F I, 70 \mathrm{eV})$

147 (2), 131 (17), 118 (6), 117 (100), 74 (3), 58 (7)

Analysis: $\quad \mathrm{C}_{7} \mathrm{H}_{14} \mathrm{O}_{3}(124.19)$
Calcd:
C, 57.51;
$\mathrm{H}, 9.65 \%$
Found:
C, 57.41;
$\mathrm{H}, 9.62 \%$

Following General Procedure II, 2-butanone (2l) (180 $\mu \mathrm{L}, 2.0 \mathrm{mmol})$, trichlorosilyl ketene acetal 1 (380 $\mu \mathrm{L}, 2.4 \mathrm{mmol}, 1.2$ equiv) and bis- $N$-oxide $(P)-(R, R)-19$ (102 mg, $0.2 \mathrm{mmol}$, 0.1 equiv) were stirred at $-20{ }^{\circ} \mathrm{C}$ for $12 \mathrm{~h}$. Work-up provided $402 \mathrm{mg}$ of crude product. Analytically pure product (224 mg, 84\%) was obtained as a colorless liquid after bulb-to-bulb distillation.

Data for (-)-31:

GC: $\quad$ major, $t_{\mathrm{R}} 7.72 \min (66.1 \%)$; minor, $t_{\mathrm{R}} 8.14 \min (33.9 \%)$ (Chiraldex GTA, $64{ }^{\circ} \mathrm{C}$, 20 psi)

Opt. Rot.: $\quad[\alpha]_{D}^{24}-1.2(c=3.08$, EtOH $)$

Analysis: $\quad \mathrm{C}_{7} \mathrm{H}_{14} \mathrm{O}_{3}$ (124.19)

$\begin{array}{lll}\text { Calcd: } & \mathrm{C}, 57.51 ; & \mathrm{H}, 9.65 \% \\ \text { Found: } & \mathrm{C}, 57.18 ; & \mathrm{H}, 9.85 \%\end{array}$


Methyl 3-hydroxy-3-methyl-5-phenylpentanoate (( ()$-3 m)$

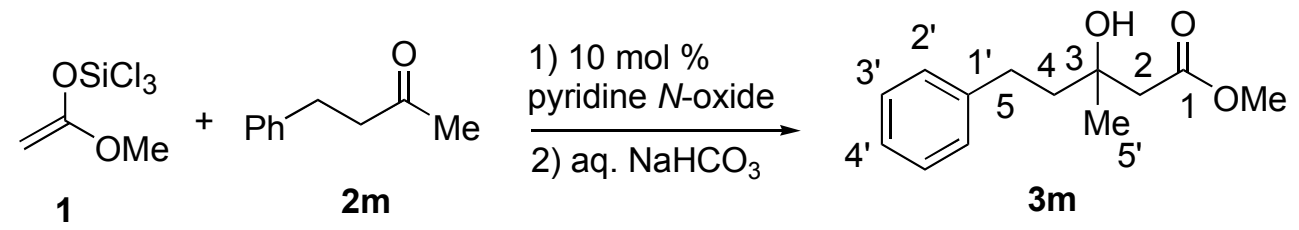

Following General Procedure I, methyl hydrocinnamyl ketone (2m) (300 $\mu \mathrm{L}, 2.0$ mmol), trichlorosilyl ketene acetal 1 (380 $\mu \mathrm{L}, 2.4 \mathrm{mmol}, 1.2$ equiv) and pyridine $N$-oxide (19.0 mg, 0.2 mmol, 0.1 equiv) were stirred at ambient temperature for $2 \mathrm{~h}$. Work-up provided $505 \mathrm{mg}$ of crude product. Analytically pure product (400 mg, 94\%) was obtained as a colorless liquid after bulb-to-bulb distillation.

Data for $( \pm)-3 m$ :

bp: $\quad 110^{\circ} \mathrm{C} \operatorname{ABT}(0.2 \mathrm{mmHg})$

${ }^{1} \mathrm{H}$ NMR: $\quad\left(400 \mathrm{MHz}, \mathrm{CDCl}_{3}\right)$

7.17-7.30 (m, $5 \mathrm{H}$, Aryl), 3.72 (s, $\left.3 \mathrm{H}, \mathrm{OCH}_{3}\right) ; 3.55$ (s, $\left.1 \mathrm{H}, \mathrm{OH}\right) ; 2.69-2.74$ (m, 2 $\mathrm{H}, \mathrm{H}_{2} \mathrm{C}(5)$ ); 2.55 (AB quartet, $\left.J=15.7,2 \mathrm{H}, \mathrm{H}_{2} \mathrm{C}(2)\right)$; 1.78-1.88 (m, $2 \mathrm{H}$, $\left.\mathrm{H}_{2} \mathrm{C}(4)\right) ; 1.32$ (s, $\left.3 \mathrm{H}, \mathrm{H}_{3} \mathrm{C}\left(6^{\prime}\right)\right)$

${ }^{13} \mathrm{C} \mathrm{NMR}: \quad\left(100 \mathrm{MHz}, \mathrm{CDCl}_{3}\right)$

$173.4(\mathrm{C}(1)), 142.2\left(\mathrm{C}\left(1^{\prime}\right)\right), 129.4\left(\mathrm{C}\left(3^{\prime}\right)\right), 129.3$ (C(2')), $125.8\left(\mathrm{C}\left(4^{\prime}\right)\right), 70.7(\mathrm{C}(3))$, $51.7\left(\mathrm{C}\left(6^{\prime}\right)\right), 44.8(\mathrm{C}(5)$ or $\mathrm{C}(2)), 43.8(\mathrm{C}(5)$ or $\mathrm{C}(2)), 30.2(\mathrm{C}(4)), 26.7\left(\mathrm{C}\left(5^{\prime}\right)\right)$

IR: (neat)

3506 (s), 3.63 (w), 3027 (m), 2952 (s), $2365(\mathrm{w}), 1732$ (s), 1603 (w), 1496 (w), 1454 (s), 1439 (s), 1377 (s), 1341 (s), 1215 (s), 1178 (s), 926 (m)

TLC: $\quad R_{f} 0.29$ (hexane/EtOAc, 5/1)

GC: $\quad t_{\mathrm{R}} 10.79 \min (50.1 \%) ; t_{\mathrm{R}} 11.18 \min (49.9 \%)\left(\right.$ Chiraldex GTA, $\left.130{ }^{\circ} \mathrm{C}, 18 \mathrm{psi}\right)$

MS: (FI, $70 \mathrm{ev)}$

223 (17), 222 (100), 187 (5), 117 (3)

(EI, $70 \mathrm{ev})$

204 (65), 144 (70), 131 (100), 117 (33), 105 (24), 91 (95), 65 (12)

Analysis: $\quad \mathrm{C}_{13} \mathrm{H}_{18} \mathrm{O}_{3}(222.23)$ 

Calcd:
C, 70.25;
$\mathrm{H}, 8.16 \%$
Found:
C, 70.16;
$\mathrm{H}, 8.00 \%$

Following General Procedure II, methyl hydrocinnamyl ketone (2m) (300 $\mu \mathrm{L}, 2.0$ mmol), trichlorosilyl ketene acetal $1(380 \mu \mathrm{L}, 2.4 \mathrm{mmol}, 1.2$ equiv) and bis- $N$-oxide $(P)-(R, R)-26(126$ $\mathrm{mg}, 0.2 \mathrm{mmol}, 0.1$ equiv) were stirred at $-20{ }^{\circ} \mathrm{C}$ for $12 \mathrm{~h}$. Work-up provided $515 \mathrm{mg}$ of crude product. Analytically pure product (413 mg, 97\%) was obtained as a colorless liquid after bulbto-bulb distillation.

Data for (+)-3m:

GC: $\quad \operatorname{minor}, t_{\mathrm{R}} 10.71 \min (32.5 \%)$; major, $t_{\mathrm{R}} 11.08 \min (67.5 \%)$ (Chiraldex GTA, 130 $\left.{ }^{\circ} \mathrm{C}, 18 \mathrm{psi}\right)$

Opt. Rot.: $[\alpha]_{\mathrm{D}}^{24}+0.4(c=2.32, \mathrm{EtOH})$

Analysis: $\quad \mathrm{C}_{13} \mathrm{H}_{18} \mathrm{O}_{3}(222.23)$
Calcd:
C, 70.25;
$\mathrm{H}, 8.16 \%$
Found:
C, 70.20;
$\mathrm{H}, 8.24 \%$

\section{Methyl 3-Cyclopropyl-3-hydroxybutanoate (( \pm )-3n)}

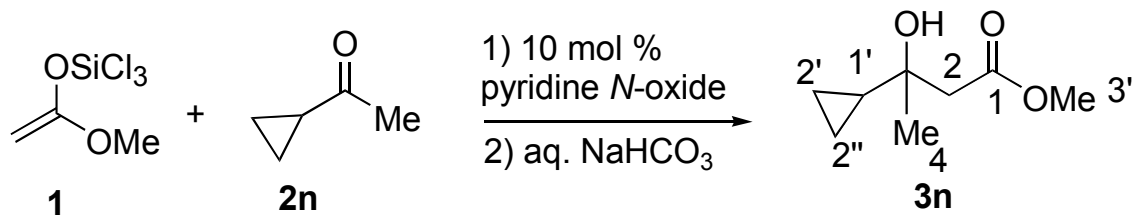

Following General Procedure II, methyl cyclopropyl ketone (2n) (200 $\mu \mathrm{L}, 2.0$ mmol), trichlorosilyl ketene acetal $1(380 \mu \mathrm{L}, 2.4 \mathrm{mmol}, 1.2$ equiv) and pyridine $N$-oxide (19.0 mg, 0.2 mmol, 0.1 equiv) were stirred at ambient temperature for $2 \mathrm{~h}$. Work-up provided $350 \mathrm{mg}$ of crude product. Analytically pure product $(278 \mathrm{mg}, 87 \%)$ was obtained as a colorless liquid after bulb-to-bulb distillation.

Data for ( \pm$)-\mathbf{3 n}$ :

bp: $\quad 90^{\circ} \mathrm{C} \mathrm{ABT}(10 \mathrm{mmHg})$

${ }^{1}$ H NMR: $\quad\left(400 \mathrm{MHz}, \mathrm{CDCl}_{3}\right)$ 
$3.72\left(\mathrm{~s}, 3 \mathrm{H}, \mathrm{OCH}_{3}\right) ; 3.27(\mathrm{bs}, 1 \mathrm{H}, \mathrm{OH}) ; 2.55(\mathrm{AB}$ quartet, $J=15.1,2 \mathrm{H}$, $\left.\mathrm{H}_{2} \mathrm{C}(2)\right)$; 1.23 (s, $\left.3 \mathrm{H}, \mathrm{H}_{3} \mathrm{C}\left(1^{\prime}\right)\right)$; 0.85-0.92 (m, $\left.1 \mathrm{H}, \mathrm{HC}\left(1^{\prime}\right)\right)$; 0.33-0.44 (m, 4 H, $\mathrm{H}_{2} \mathrm{C}\left(2^{\prime}\right)$ and $\left.\mathrm{H}_{2} \mathrm{C}\left(2^{\prime \prime}\right)\right)$

${ }^{13} \mathrm{C}$ NMR: $\quad\left(100 \mathrm{MHz}, \mathrm{CDCl}_{3}\right)$

$173.6(\mathrm{C}(1)), 69.4(\mathrm{C}(3)), 51.9\left(\mathrm{C}\left(3^{\prime}\right)\right), 24.1(\mathrm{C}(2)), 27.2\left(\mathrm{C}\left(1^{\prime}\right)\right), 21.0(\mathrm{C}(4)), 0.9$ $\left(\mathrm{C}\left(2^{\prime}\right.\right.$ or $\left.\left.2^{\prime \prime}\right)\right), 0.2\left(\mathrm{C}\left(2^{\prime}\right.\right.$ or $\left.\left.2^{\prime \prime}\right)\right)$

IR: (neat)

3510 (s), 3086 (w), 3007 (s), 2977 (s), 1719 (s), 1438 (s), 1375 (m), 1349 (s), 1202 (s), 1050 (m), 1017 (m), 947 (m)

TLC: $\quad R_{f} 0.40$ (hexane/EtOAc, 5/1)

GC: $\quad t_{\mathrm{R}} 4.97 \min (49.9 \%) ; t_{\mathrm{R}} 5.21 \min (50.1 \%)$ (Chiraldex GTA, $\left.80{ }^{\circ} \mathrm{C}, 20 \mathrm{psi}\right)$

MS: $\quad(\mathrm{FI}, 70 \mathrm{ev})$

159 (4), 158 (31), 144 (8), 143 (100), 117 (3), 84 (3), 74 (5)

(EI, $70 \mathrm{ev})$

143 (80), 130 (10), 117 (25), 98 (25), 85 (100), 69 (70), 56 (18)

Analysis: $\quad \mathrm{C}_{8} \mathrm{H}_{14} \mathrm{O}_{3}(158.20)$

Calcd: $\quad$ C, $60.74 ; \quad H, 8.92 \%$

Found: $\quad \mathrm{C}, 60.99 ; \quad \mathrm{H}, 8.95 \%$

Following General Procedure II, methyl cyclopropyl ketone (2n) (200 $\mu \mathrm{L}, 2.0$ mmol), trichlorosilyl ketene acetal $1(380 \mu \mathrm{L}, 2.4 \mathrm{mmol}, 1.2$ equiv) and bis- $N$-oxide $(P)-(R, R)-19(102$ $\mathrm{mg}, 0.2 \mathrm{mmol}, 0.1$ equiv) were stirred at $-20{ }^{\circ} \mathrm{C}$ for $12 \mathrm{~h}$. Work-up provided $470 \mathrm{mg}$ of crude product. Analytically pure product $(279 \mathrm{mg}, 84 \%)$ was obtained as a colorless liquid after bulbto-bulb distillation.

Data for (-)-3n:

GC: $\operatorname{minor}, t_{\mathrm{R}} 4.92 \min (39.9 \%)$; major, $t_{\mathrm{R}} 5.15 \min (60.1 \%)$ (Chiraldex GTA, $80{ }^{\circ} \mathrm{C}$, 20 psi)

Opt. Rot.: $\quad[\alpha]_{\mathrm{D}}^{24}-0.7(c=4.54, \mathrm{EtOH})$ 
Analysis: $\quad \mathrm{C}_{8} \mathrm{H}_{14} \mathrm{O}_{3}(158.20)$
Calcd:
C, 60.74;
$\mathrm{H}, 8.92 \%$
Found:
C, 60.24;
H, $9.02 \%$

\section{Methyl 3-Cyclohexyl-3-hydroxybutanoate ((土)-3o)}

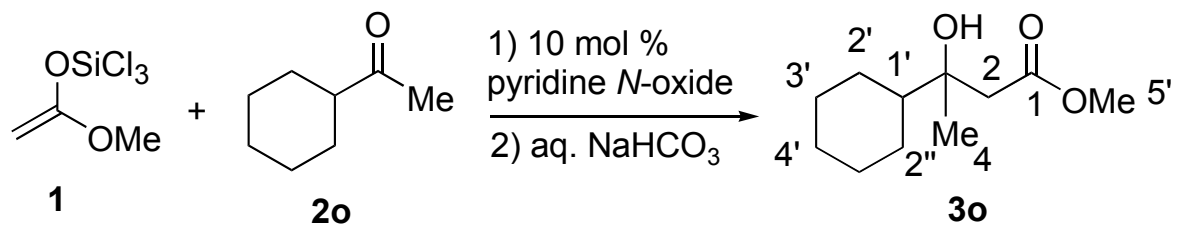

Following General Procedure I, methyl cyclohexyl ketone (2o) (290 $\mu \mathrm{L}, 2.0 \mathrm{mmol})$, trichlorosilyl ketene acetal 1 (380 $\mu \mathrm{L}, 2.4 \mathrm{mmol}, 1.2$ equiv) and pyridine $N$-oxide (19.0 mg, 0.2 mmol, 0.1 equiv) were stirred at ambient temperature for $2 \mathrm{~h}$. Work-up provided $503 \mathrm{mg}$ of crude product. Analytically pure product $(376 \mathrm{mg}, 94 \%)$ was obtained as a colorless liquid after bulb-to-bulb distillation.

Data for $( \pm)-\mathbf{3 o}$ :

bp: $\quad 110^{\circ} \mathrm{C}$ ABT $(0.3 \mathrm{mmHg})$

${ }^{1} \mathrm{H}$ NMR: $\quad\left(400 \mathrm{MHz}, \mathrm{CDCl}_{3}\right)$

3.77(s, 3H, $\left.\mathrm{OCH}_{3}\right) ; 3.44$ (s, 1H, OH); 2.50 (AB quartet, $\left.J=15.4,2 \mathrm{H}, \mathrm{H}_{2} \mathrm{C}(2)\right)$;

0.95-1.89 (m, 11H, cyclohexyl), 1.16 (s, 3H, $\left.\mathrm{H}_{3} \mathrm{C}(4)\right)$

${ }^{13} \mathrm{C} \mathrm{NMR}: \quad\left(100 \mathrm{MHz}, \mathrm{CDCl}_{3}\right)$

$173.8(\mathrm{C}(1)), 73.0(\mathrm{C}(3)), 51.6\left(\mathrm{C}\left(5^{\prime}\right)\right), 47.9$ (C(2)), 42.7 (C(1')), $27.3(\mathrm{C}(4)), 26.9$

$\left(\mathrm{C}\left(2^{\prime}\right.\right.$ or $\left.\left.2^{\prime \prime}\right)\right), 26.6\left(\mathrm{C}\left(2^{\prime}\right.\right.$ or $\left.\left.2^{\prime \prime}\right)\right), 26.6\left(\mathrm{C}\left(3^{\prime}\right)\right), 26.4\left(\mathrm{C}\left(3^{\prime}\right)\right), 23.7\left(\mathrm{C}\left(4^{\prime}\right)\right)$.

IR: (neat)

3529 (s), 2923 (s), 2954 (s), 1720 (s), 1438 (s), 1378 (s), 1332 (s), 1240 (m), 1117

(m), 1007 (m), $943(\mathrm{w})$

TLC: $\quad$ Rf 0.45 (hexane/EtOAc, 5/1)

GC: $t_{\mathrm{R}} 10.74 \min (49.6 \%) ; t_{\mathrm{R}} 11.20 \min (50.4 \%)\left(\right.$ Chiraldex GTA, $\left.102{ }^{\circ} \mathrm{C}, 18 \mathrm{psi}\right)$

MS: (FI, $70 \mathrm{ev})$

201 (3), 182 (5), $118(5), 117$ (100), 83 (18) 
(EI, $70 \mathrm{ev})$

185 (2), 151 (2), 127 (8), 117 (100), 85 (40), 67 (5), 55 (13)

Analysis: $\mathrm{C}_{11} \mathrm{H}_{20} \mathrm{O}_{3}(200.23)$
Calcd:
C, 65.97;
H, $10.06 \%$
Found:
C, 65.81;
H, 9.99\%

Following General Procedure II, methyl cyclohexyl ketone (2o) (290 $\mu \mathrm{L}, 2.0$ mmol), trichlorosilyl ketene acetal $1(380 \mu \mathrm{L}, 2.4 \mathrm{mmol}, 1.2$ equiv) and bis- $N$-oxide $(P)-(R, R)-19(102$ $\mathrm{mg}, 0.2 \mathrm{mmol}, 0.1$ equiv) were stirred at $-20{ }^{\circ} \mathrm{C}$ for $12 \mathrm{~h}$. Work-up provided $503 \mathrm{mg}$ of crude product. Analytically pure product $(367 \mathrm{mg}, 91 \%)$ was obtained as a colorless liquid after bulbto-bulb distillation.

Data for (-)-30:

GC: $\quad$ major, $t_{\mathrm{R}} 6.10 \min (66.0 \%)$; minor, $t_{\mathrm{R}} 10.61 \min (34.0 \%)$ (Chiraldex GTA, 102 $\left.{ }^{\circ} \mathrm{C}, 18 \mathrm{psi}\right)$

Opt. Rot.: $\quad[\alpha]_{\mathrm{D}}^{24}-3.1(c=2.69, \mathrm{EtOH})$

Analysis: $\quad \mathrm{C}_{11} \mathrm{H}_{20} \mathrm{O}_{3}(200.23)$
Calcd:
C, 65.97;
H, $10.06 \%$
Found:
C, 65.54;
$\mathrm{H}, 9.95 \%$

\section{Methyl 3-Hydroxy-3,4,4-trimethylpentanoate (( \pm )-3p)}

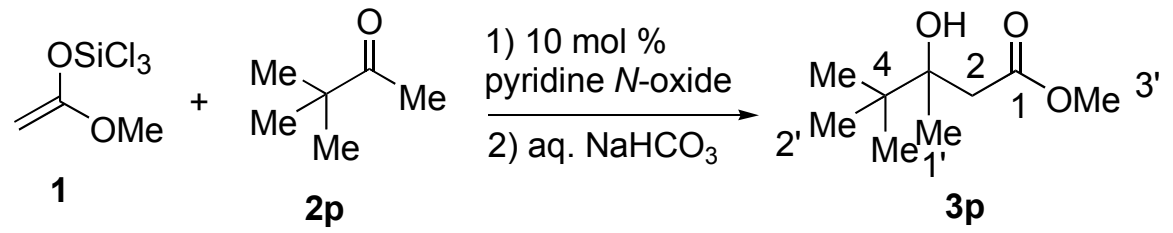

Following General Procedure I, pinacolone (2p) $(260 \mu \mathrm{L}, 2.0 \mathrm{mmol})$, trichlorosilyl ketene acetal 1 (380 $\mu \mathrm{L}, 2.4 \mathrm{mmol}, 1.2$ equiv) and pyridine $N$-oxide (19.0 mg, $0.2 \mathrm{mmol}, 0.1$ equiv) were stirred at ambient temperature for $8 \mathrm{~h}$. Work-up provided $360 \mathrm{mg}$ of crude product. Analytically pure product (336 mg, 93\%) was obtained as a colorless liquid after bulb-to-bulb distillation. 


\section{Data for $( \pm)-\mathbf{3 p}$ :}

bp: $\quad 100^{\circ} \mathrm{C} \mathrm{ABT}(3 \mathrm{mmHg})$

${ }^{1}$ H NMR: $\quad\left(400 \mathrm{MHz}, \mathrm{CDCl}_{3}\right)$

3.72 (s, $\left.3 \mathrm{H}, \mathrm{OCH}_{3}\right) ; 3.49$ (bs, $\left.1 \mathrm{H}, \mathrm{OH}\right) ; 2.50$ (AB quartet, $J=15.1,2 \mathrm{H}, \mathrm{H}_{2} \mathrm{C}(2)$ );

1.21 (s, $\left.3 \mathrm{H}, \mathrm{H}_{3} \mathrm{C}\left(1^{\prime}\right)\right) ; 0.53$ (s, $\left.9 \mathrm{H}, 3 \mathrm{H}_{3} \mathrm{C}\left(2^{\prime}\right)\right)$

${ }^{13} \mathrm{C} \mathrm{NMR}: \quad\left(100 \mathrm{MHz}, \mathrm{CDCl}_{3}\right)$

174.6 (C(1)), $74.8(\mathrm{C}(3)), 51.7\left(\mathrm{C}\left(3^{\prime}\right)\right), 40.4(\mathrm{C}(2)), 37 . \mathrm{X}(\mathrm{C}(4)), 25.1\left(\mathrm{C}\left(1^{\prime}\right)\right), 22.2$ $\left(\mathrm{C}\left(2^{\prime}\right)\right)$

IR: (neat)

3392 (s), 2948 (s), 3935 (s), 1719 (m), 1656 (m), 1449 (m), 1200 (m), 1113 (m), $1026(\mathrm{~s})$

TLC: $R_{f} 0.45$ (hexane/EtOAc, 5/1)

GC: $\quad t_{\mathrm{R}} 3.22 \mathrm{~min}(49.9 \%) ; t_{\mathrm{R}} 3.40 \mathrm{~min}(50.1 \%)$ (Chiraldex GTA, $\left.85^{\circ} \mathrm{C}, 20 \mathrm{psi}\right)$

MS: (EI, $70 \mathrm{eV}$ )

157 (15), 141 (12), 125 (20), 117 (70), 85 (100), 75 (13), 57 (75)

Analysis: $\quad \mathrm{C}_{9} \mathrm{H}_{18} \mathrm{O}_{3}(174.24)$

Calcd: $\quad$ C, $62.04 ; \quad \mathrm{H}, 10.41 \%$

Found: $\quad$ C, $62.25 ; \quad \mathrm{H}, 10.31 \%$

Following General Procedure II, pinacolone (2p) $(260 \mu \mathrm{L}, 2.0 \mathrm{mmol})$, trichlorosilyl ketene acetal 1 (380 $\mu \mathrm{L}, 2.4 \mathrm{mmol}, 1.2$ equiv) and bis- $N$-oxide $(P)-(R, R)-19(102 \mathrm{mg}, 0.2 \mathrm{mmol}$, 0.1 equiv) were stirred at $-20{ }^{\circ} \mathrm{C}$ for $32 \mathrm{~h}$. Work-up provided $455 \mathrm{mg}$ of crude product. Analytically pure product ( $315 \mathrm{mg}, 87 \%$ ) was obtained as a colorless liquid after bulb-to-bulb distillation.

Data for $(-)-3 \mathbf{p}$ :

GC: $\quad \operatorname{minor}, t_{\mathrm{R}} 4.26 \min (29.5 \%)$; major, $t_{\mathrm{R}} 4.55 \min (71.5 \%)$ (Chiraldex GTA, $80{ }^{\circ} \mathrm{C}$, $20 \mathrm{psi})$

Opt. Rot.: $[\alpha]_{\mathrm{D}}^{24}-4.9(c=0.65, \mathrm{EtOH})$ 
Analysis: $\quad \mathrm{C}_{9} \mathrm{H}_{18} \mathrm{O}_{3}(174.24)$
Calcd:
C, 62.04;
$\mathrm{H}, 10.41 \%$
Found:
C, 61.89;
$\mathrm{H}, 10.40 \%$

\section{Methyl (1-Hydroxy-1,2,3,4-tetrahydro-2-naphthalenyl)acetate (( \pm )-3q)}

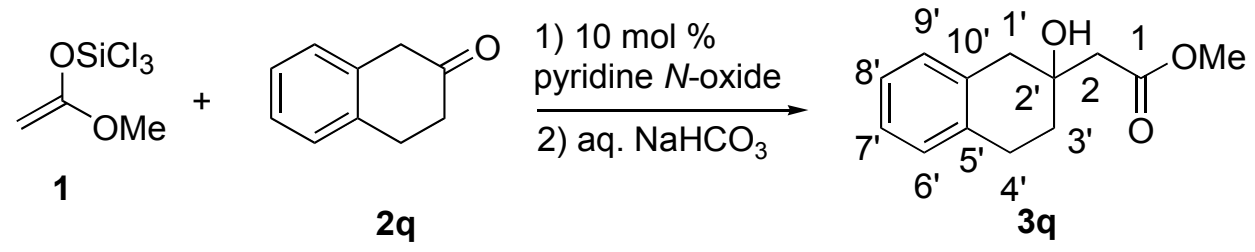

Following General Procedure II, 2-tetralone (2q) (265 $\mu \mathrm{L}, 2.0 \mathrm{mmol})$, trichlorosilyl ketene acetal 1 (380 $\mu \mathrm{L}, 2.4 \mathrm{mmol}, 1.2$ equiv) and pyridine $N$-oxide $(19.0 \mathrm{mg}, 0.2 \mathrm{mmol}, 0.1$ equiv) were stirred at ambient temperature for $2 \mathrm{~h}$. Work-up provided $402 \mathrm{mg}$ mixture of the product and 2-tetralone which was purified by silica gel column chromatography (hexane/EtOAc 5/1). Analytically pure product ( $201 \mathrm{mg}, 45 \%$ ) was obtained after bulb-to-bulb distillation.

\section{Data for ( \pm -)-3q:}

bp: $\quad 110^{\circ} \mathrm{C}$ ABT $(0.2 \mathrm{mmHg})$

${ }^{1} \mathrm{H}$ NMR: $\quad\left(400 \mathrm{MHz}, \mathrm{CDCl}_{3}\right)$

7.04-7.13 (m, 5 H, Aryl); 3.75 (s, 3 H, OCH3); 3.63 (bs, 1 H, OH); 3.04 (dt, J = 14.7, 6.3, $1 \mathrm{H}, \mathrm{HeqC}\left(3^{\prime}\right)$ ); 2.91 (AB quartet, $J=16.6,2 \mathrm{H}, \mathrm{H}_{2} \mathrm{C}(2)$ ); 2.77 (dt, $J=$ 17.1, 6.3, $\left.1 \mathrm{H}, \mathrm{H}_{a x} \mathrm{C}\left(3^{\prime}\right)\right) ; 2.60$ (s, $2 \mathrm{H}, \mathrm{H}_{2} \mathrm{C}\left(1^{\prime}\right)$ ); 1.98 (ddd, $J=8.0$, 6.3, 1.5, $\left.\mathrm{H}_{\mathrm{eq}} \mathrm{C}\left(4^{\prime}\right)\right) ; 1.84$ (ddd, $\left.J=7.9,6.3,0.9, \mathrm{H}_{\mathrm{ax}} \mathrm{C}\left(4^{\prime}\right)\right)$

${ }^{13} \mathrm{C} \mathrm{NMR}: \quad\left(100 \mathrm{MHz}, \mathrm{CDCl}_{3}\right)$

$173.3(\mathrm{C}(1)), 135.0\left(\mathrm{C}\left(10^{\prime}\right)\right), 133.9\left(\mathrm{C}\left(5^{\prime}\right)\right), 129.5\left(\mathrm{C}\left(8^{\prime}\right)\right), 129.6\left(\mathrm{C}\left(7^{\prime \prime}\right)\right), 126.0$ $\left(\mathrm{C}\left(9^{\prime}\right.\right.$ or $\left.\left.6^{\prime}\right)\right), 125.9\left(\mathrm{C}\left(9^{\prime}\right.\right.$ or $\left.\left.\left.6^{\prime}\right)\right), 69.4\left(\mathrm{C}\left(2^{\prime}\right)\right), 51.8\left(\mathrm{OCH}_{3}\right)\right), 43.7(\mathrm{C}(2)), 41.7$ $\left(\mathrm{C}\left(1^{\prime}\right)\right), 34.1\left(\mathrm{C}\left(4^{\prime}\right)\right), 34.0\left(\mathrm{C}\left(3^{\prime}\right)\right)$

IR: (neat)

3392 (s), 2948 (s), 2935 (s), 1719 (m), 1656 (m), 1449 (m), 1200 (m), 1113 (m), $1026(\mathrm{~s})$ 
TLC: $R_{f} 0.29$ (hexane/EtOAc, 5/1)

MS: (EI, $70 \mathrm{eV}$ ) 202 (35), 147 (100), 129 (65), 115 (13), 103 (5), 91 (35), 77 (5)

Analysis: $\quad \mathrm{C}_{13} \mathrm{H}_{16} \mathrm{O}_{3}(220.27)$
Calcd:
C, 70.88;
$\mathrm{H}, 7.32 \%$
Found:
C, 70.80;
$\mathrm{H}, 7.37 \%$

\section{General Procedure III. Preparation of $\boldsymbol{N}$-Oxides from Bipyridines}

3-Chloroperoxybenzoic acid ( $m$ CPBA) was added to a solution of the bipyridine in $\mathrm{CH}_{2} \mathrm{Cl}_{2}$ and the mixture was stirred overnight (about $12 \mathrm{~h}$ ) at rt. After the pyridine starting material was consumed, cold, $40 \%$ aqueous $\mathrm{KOH}$ solution was added into the flask and the resulting slurry was stirred for $1 \mathrm{~h}$ at $\mathrm{rt}$. The mixture was diluted with water and thoroughly extracted with $\mathrm{CH}_{2} \mathrm{Cl}_{2}$. The combined organic layers were dried over $\mathrm{MgSO}_{4}$ and were concentrated under reduced pressure. The residue was further purified by flash column chromatography.

\section{Preparation of $(R, R)-6,6$ '-Bis-(1-methoxy-2,2-dimethylpropyl)-2,2'-bipyridine Bis- $N$-oxide $((R, R)-17)$}

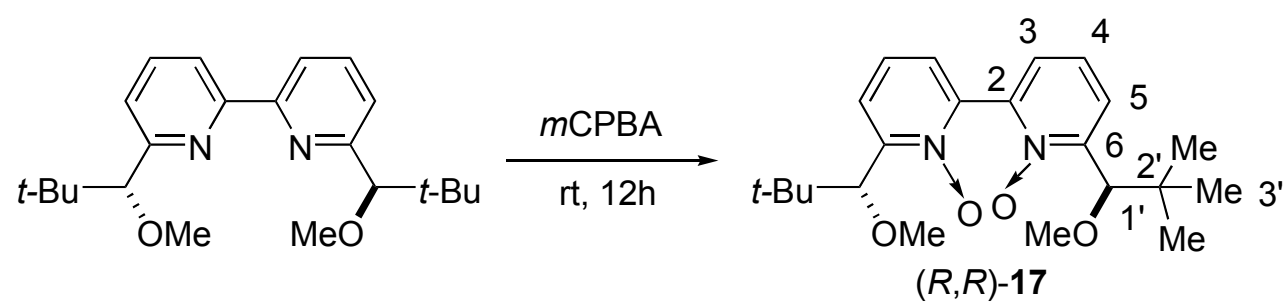

Following General Procedure III, $(R, R)$-6,6'-bis-(1-methoxy-2,2-dimethylpropyl)-2,2'bipyridine (390 mg, $1.1 \mathrm{mmol}$ ) and $m \mathrm{CPBA}(570 \mathrm{mg}, 3.3 \mathrm{mmol}, 3.0$ equiv) were dissolved in $\mathrm{CH}_{2} \mathrm{Cl}_{2}(5 \mathrm{~mL})$ and the mixture were stirred for $12 \mathrm{~h}$ at $\mathrm{rt}$. Work-up provided $607 \mathrm{mg}$ of white solid residue. Silica gel column chromatography $\left(\mathrm{CH}_{2} \mathrm{Cl}_{2} / \mathrm{MeOH}, 19 / 1\right)$ provided $425 \mathrm{mg}(95 \%)$ of white needle crystals. An analytically pure sample was obtained after recrystallization from hexane. 


\section{Data for $(R, R)-\mathbf{1 7}$ :}

mp: $\quad 185-186^{\circ} \mathrm{C}$ (hexane)

${ }^{1}$ H NMR: $\quad\left(400 \mathrm{MHz}, \mathrm{CDCl}_{3}\right)$

7.47 (dd, $J=7.8,1.9,2$ H, HC(3)), 7.44 (dd, $J=7.8,2.2,2$ H, H(C(5)), 7.29 (t, $J$ = 7.8, $\left.2 \mathrm{H}, \mathrm{HC}(4)), 5.04\left(\mathrm{~s}, 2 \mathrm{H}, \mathrm{HC}\left(1^{\prime}\right)\right), 3.24\left(\mathrm{~s}, 6 \mathrm{H}, \mathrm{OCH}_{3}\right)\right), 0.99(\mathrm{~s}, 18 \mathrm{H}$, $\left.\mathrm{H}_{3} \mathrm{C}\left(3^{\prime}\right)\right)$

${ }^{13} \mathrm{C}$ NMR: $\quad\left(100 \mathrm{MHz}, \mathrm{CDCl}_{3}\right)$

$151.8(\mathrm{C}(2$ or 6$)), 143.7$ ( $\mathrm{C}(2$ or 6$)), 126.1$ (C(3 or 5$)), 125.7$ (C(3 or 5$)), 123.3$ $(\mathrm{C}(4)), 82.5\left(\left(\mathrm{OCH}_{3}\right)\right), 58.2\left(\mathrm{C}\left(1^{\prime}\right)\right), 37.4\left(\mathrm{C}\left(2^{\prime}\right)\right), 25.7\left(\mathrm{C}\left(3^{\prime}\right)\right)$

IR: $(\mathrm{KBr})$

2972 (s), 2904 (s), 2977 (m), 2921 (s), 1629 (w), 1477 (m), 1398 (s), 1382 (s), 1330 (s), 1297 (s), 1245 (s), 1207 (m), 1162 (s), 1100 (s), 1045 (w), 968 (m), 850 (s), $800(\mathrm{~m}), 773(\mathrm{~m})(\mathrm{w})$

TLC: $\quad R_{f} 0.34\left(\mathrm{CH}_{2} \mathrm{Cl}_{2} / \mathrm{MeOH}, 19 / 1\right)$

MS: (FAB)

390 (24), 389 (100), 374 (10), 373 (40), 357 (24), 119 (14)

Opt. Rot.: $[\alpha]_{\mathrm{D}}^{24}+126.0(c=0.89, \mathrm{EtOH})$

Analysis: $\quad \mathrm{C}_{22} \mathrm{H}_{32} \mathrm{O}_{2} \mathrm{~N}_{2} \mathrm{O}_{2}(388.51)$
Calcd:
C, 68.01;
H, 8.30;
$\mathrm{N}, 7.21 \%$
Found:
C, 68.09;
H, 8.24;
$\mathrm{N}, 7.27 \%$ 
$(R, R)-6,6$ '-Bis-(1-butoxy-2,2-dimethylpropyl)-2,2'-bipyridine Bis- $N$-oxide $((R, R)-18)$

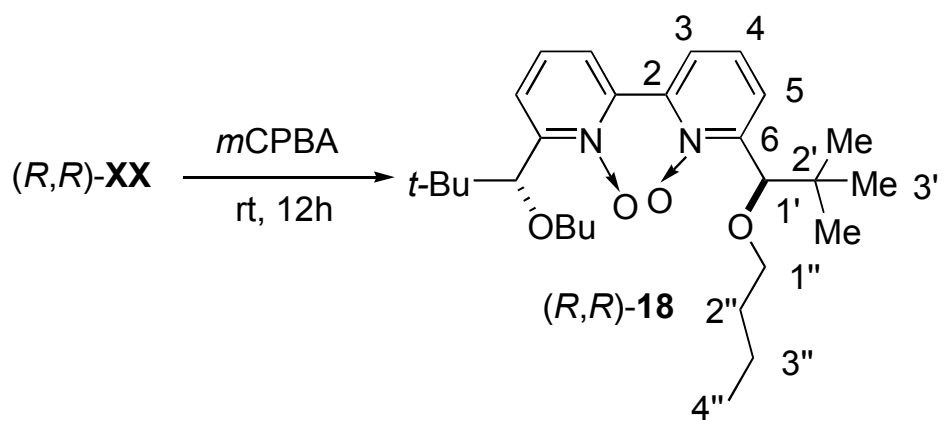

Following General Procedure III, bipyridine $(R, R)-\mathbf{X X}(160 \mathrm{mg}, 0.36 \mathrm{mmol})$ and $m \mathrm{CPBA}$ (200 mg, $1.2 \mathrm{mmol}, 3.4$ equiv) were dissolved in $\mathrm{CH}_{2} \mathrm{Cl}_{2}(2 \mathrm{~mL})$ and the mixture was stirred overnight at rt. Work-up provided $177 \mathrm{mg}$ of white solid residue. Purification by silica gel column chromatography (hexane/EtOAc, 3/1) provided $(167 \mathrm{mg}, 97 \%$ ) of the product as white needles. An analytically pure sample was obtained after recrystallization from hexane.

Data for $(R, R)-\mathbf{1 8}$ :

mp: $\quad 157-158^{\circ} \mathrm{C}$ (hexane)

${ }^{1} \mathrm{H}$ NMR: $\quad\left(400 \mathrm{MHz}, \mathrm{CDCl}_{3}\right)$

$7.48(\mathrm{dd}, J=7.8,2.2,2 \mathrm{H}, \mathrm{HC}(3)), 7.42(\mathrm{dd}, J=7.5,1.9,2 \mathrm{H}, \mathrm{HC}(5)), 7.27(\mathrm{t}, J=$ 7.8, 2 H, H(C(4)), 5.08 (s, 2 H, HC(1')), 3.25-3.44 (m, 4H, $\left.\mathrm{H}_{2} \mathrm{C}\left(1^{\prime \prime}\right)\right), 1.47-1.58$ (m, $\left.4 \mathrm{H}, \mathrm{H}_{2} \mathrm{C}\left(2^{\prime \prime}\right)\right), 1.30-1.40\left(\mathrm{~m}, 4 \mathrm{H}, \mathrm{H}_{2} \mathrm{C}\left(3^{\prime \prime}\right)\right), 0.98$ (s, 18H, $\left.\mathrm{H}_{3} \mathrm{C}\left(3^{\prime}\right)\right), 0.88$ (t, $J=$ $\left.7.3,6 \mathrm{H}, \mathrm{H}_{3} \mathrm{C}\left(4^{\prime \prime}\right)\right)$

${ }^{13} \mathrm{C}$ NMR: $\quad\left(100 \mathrm{MHz}, \mathrm{CDCl}_{3}\right)$

$152.5(\mathrm{C}(2$ or 6$)), 143.6(\mathrm{C}(2$ or 6$)), 126.0(\mathrm{C}(3$ or 5$)), 125.9(\mathrm{C}(3$ or 5$)), 123.3$ (C(4)), $80.6\left(\mathrm{C}\left(1^{\prime}\right)\right), 70.1\left(\mathrm{C}\left(1^{\prime \prime}\right)\right), 37.2\left(\mathrm{C}\left(2^{\prime}\right)\right), 32.0\left(\mathrm{C}\left(2^{\prime \prime}\right)\right), 25.7\left(\mathrm{C}\left(3^{\prime}\right)\right), 19.4$ $\left(\mathrm{C}\left(3^{\prime \prime}\right)\right), 13.9\left(\mathrm{C}\left(4^{\prime \prime}\right)\right)$

IR: $\quad(\mathrm{KBr})$

2958 (s), 2933 (s), 2873 (s), 1477 (m), 1396 (s), 1378 (s), 1326 (s), 1297 (m), 1240 (s), 1201 (m), 1091 (s), 848 (m), 779 (m)

TLC: $\quad R_{f} 0.34$ (hexane/EtOAc, 3/1) 
MS: (FAB)

476 (6), 474 (32), 473 (100), 471 (5), 458 (13), 457 (40), 442 (14), 441 (41)

Opt. Rot.: $\quad[\alpha]_{\mathrm{D}}^{24}+21.5(c=0.95$, EtOH $)$

Analysis: $\quad \mathrm{C}_{28} \mathrm{H}_{44} \mathrm{~N}_{2} \mathrm{O}_{2}(472.67)$

Calcd: $\quad$ C, $76.32 ; \quad \mathrm{H}, 10.06 ; \quad \mathrm{N}, 6.36 \%$

Found: $\quad$ C, $76.51 ; \quad \mathrm{H}, 10.27 ; \quad \mathrm{N}, 6.23 \%$

Preparation of $(P)-(R, R)-5,5 '$-Bismethyl-6,6'-Bis-(1-butyloxy-2,2-dimethylpropyl))-2,2'bipyridine Bis- $N$-oxide $((P)-(R, R)-19)$

1. 1-[2-(5-Methylpyridyl)]-2,2-dimethylpropanone (32)
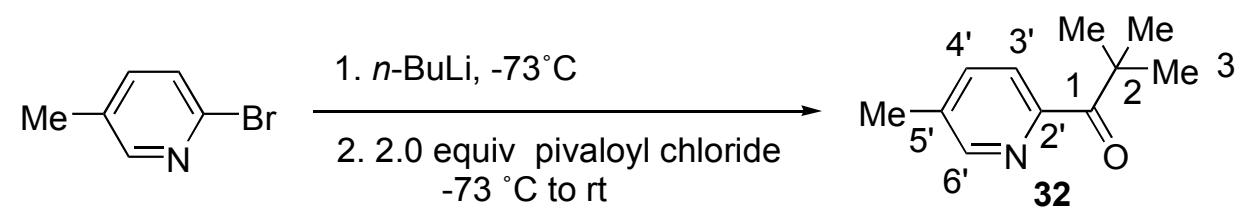

To a suspension of 2-bromo-5-methylpyridine $(5.02 \mathrm{~g}, 29.2 \mathrm{mmol})$ in $\mathrm{Et}_{2} \mathrm{O}(50 \mathrm{~mL})$ was added $n$-BuLi in hexane $\left(20.0 \mathrm{~mL}, 31 \mathrm{mmol}, 1.05\right.$ equiv) at $-73{ }^{\circ} \mathrm{C}$ dropwise over $15 \mathrm{~min}$. The mixture was stirred for $1 \mathrm{~h}$ at $-73^{\circ} \mathrm{C}$ and the resulting deep red solution was transferred dropwise via a short cannula to a rapidly stirred solution of pivaloyl chloride ( $7.2 \mathrm{~mL}, 61 \mathrm{mmol}, 2.0$ equiv) in THF $(10 \mathrm{~mL})$, which was cooled to $-73{ }^{\circ} \mathrm{C}$. After stirring for $4 \mathrm{~h}$ at $-73{ }^{\circ} \mathrm{C}$, the reddish mixture was slowly brought to rt over $5 \mathrm{~h}$ and the reaction was quenched with cold water (20 $\mathrm{mL}$ ). The mixture was pour into cold $\mathrm{NaOH}$ solution $(40 \%, 100 \mathrm{~mL})$ and stirred for $30 \mathrm{~min}$. The aqueous layer was extracted with $\mathrm{Et}_{2} \mathrm{O}(3 \times 100 \mathrm{~mL})$. The combined organic layers were dried over $\mathrm{MgSO}_{4}$ and concentrated under reduced pressure. Purification of the semi-solid residue by silica gel chromatography (hexane/EtOAc, 10/1) afforded $4.72 \mathrm{~g}$ of product 37 (90\%) as colorless oil. An analytically pure sample was obtained after bulb-to-bulb distillation.

Data for 32:

bp: $\quad 112^{\circ} \mathrm{C} \mathrm{ABT}(7.3 \mathrm{mmHg})$

${ }^{1}$ H NMR: $\quad\left(500 \mathrm{MHz}, \mathrm{CDCl}_{3}\right)$

8.43-8.44 (m, $\left.1 \mathrm{H}, \mathrm{HC}\left(5^{\prime}\right)\right), 7.82\left(\mathrm{~d}, J=8.0,1 \mathrm{H}, \mathrm{HC}\left(2^{\prime}\right)\right)$, 7.56-7.58 (m, $1 \mathrm{H}$, $\mathrm{H}\left(\mathrm{C}\left(3^{\prime}\right)\right), 2.38\left(\mathrm{~s}, 3 \mathrm{H}, \mathrm{H}_{3} \mathrm{C}\left(6^{\prime}\right)\right), 1.45\left(\mathrm{~s}, 9 \mathrm{H},\left(\mathrm{H}_{3} \mathrm{C}\right)_{3} \mathrm{C}(2)\right)$ 
${ }^{13} \mathrm{C} \mathrm{NMR}: \quad\left(100 \mathrm{MHz}, \mathrm{CDCl}_{3}\right)$

206.6 (C(1)), 152.2 (C(2')), 148.1 (C(6')), 136.9 (C(4')), 136.0 (C(5')), 129.4 $\left(\mathrm{C}\left(3^{\prime}\right)\right), 44.1\left(\mathrm{H}_{3} \mathrm{C}\left(\mathrm{C}\left(4^{\prime}\right)\right)\right), 27.5(\mathrm{C}(3)), 18.6(\mathrm{C}(2))$.

IR: (neat)

2955 (m), 2928 (m), 2867 (w),1682 (s), 1566 (w), 1481 (m), 1297 (m), 1199 (s), $964(\mathrm{~s}) 849(\mathrm{w})$

TLC: $\quad R_{f} 0.44$ (hexane/EtOAc, 10/1)

MS: (EI)

177 (14), 149 (11), 120 (10), 107 (10), 93 (100), 92 (39), 65 (18), 57 (15)

Analysis: $\quad \mathrm{C}_{11} \mathrm{H}_{15} \mathrm{NO}(177.25)$
Calcd:
C, 74.54;
H, 8.53;
$\mathrm{N}, 7.90 \%$
Found:
C, 74.55;
H, 8.68;
$\mathrm{N}, 8.04 \%$

\section{1-[2-(5-Methylpyridyl)]-2,2-dimethylpropanol (( 1$)-33)$}

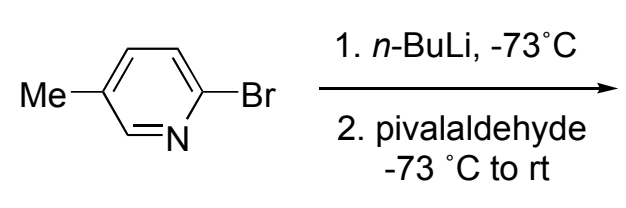

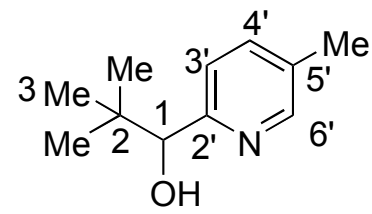

$( \pm)-33$

To a suspension of 2-bromo-5-methylpyridine (344 mg, $2.0 \mathrm{mmol})$ in $\mathrm{Et}_{2} \mathrm{O}(5 \mathrm{~mL})$ was added $n$-BuLi in hexane $\left(1.4 \mathrm{~mL}, 2.2 \mathrm{mmol}, 1.1\right.$ equiv) at $-73{ }^{\circ} \mathrm{C}$ dropwise over $15 \mathrm{~min}$. The mixture was stirred for $1 \mathrm{~h}$ at $-73{ }^{\circ} \mathrm{C}$ and to the resulting deep-red solution was added pivalaldehyde $\left(240 \mu \mathrm{L}, 2.2 \mathrm{mmol}, 1.1\right.$ equiv). The mixture was stirred for $1 \mathrm{~h}$ at $-73^{\circ} \mathrm{C}$ and then was warmed to rt over $40 \mathrm{~min}$. The reaction mixture was quenched with cold water and the aqueous layer was extracted with $\mathrm{Et}_{2} \mathrm{O}(3 \times 30 \mathrm{~mL})$. The combined organic layers were dried over $\mathrm{MgSO}_{4}$ and concentrated under reduced pressure. Purification of the semi-solid residue by silica gel chromatography (hexane/EtOAc, 5/1) afforded $331 \mathrm{mg}$ of product (91\%) as white needles.

Data for $( \pm)-33$ :

mp: $\quad 42-43^{\circ} \mathrm{C}$ (hexane) 
${ }^{1} \mathrm{H}$ NMR: $\quad\left(500 \mathrm{MHz}, \mathrm{CDCl}_{3}\right)$

8.39 (s, $\left.1 \mathrm{H}, \mathrm{HC}\left(6^{\prime}\right)\right), 7.48$ (d, J= 7.9, $\left.1 \mathrm{H}, \mathrm{HC}\left(4^{\prime}\right)\right), 7.12$ (d, J= 7.9, $1 \mathrm{H}, \mathrm{H}\left(\mathrm{C}\left(3^{\prime}\right)\right)$, 4.36 (s, $1 \mathrm{H}, \mathrm{HC}(1)), 4.31$ (bs, $1 \mathrm{H}, \mathrm{HO}), 2.35$ (s, $\left.3 \mathrm{H}, \mathrm{H}_{3} \mathrm{C}\left(6^{\prime}\right)\right), 0.91$ (s, $9 \mathrm{H}$, $\left.\left(\mathrm{H}_{3} \mathrm{C}\right)_{3} \mathrm{C}(2)\right)$

\section{1-[2-(5-Methylpyridyl)]-2,2-dimethylpropy1 Trifluoroacetate (( $( \pm)-42)$}

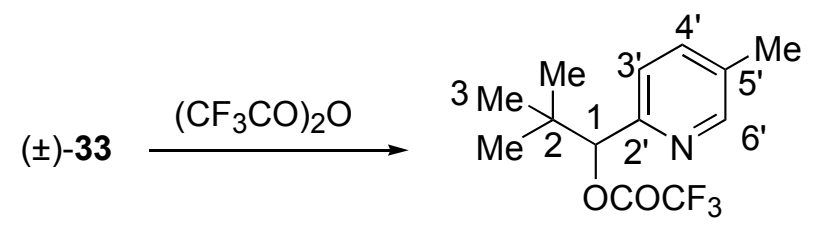

$( \pm)-42$

To a solution of $( \pm)-33(45 \mathrm{mg}, 0.25 \mathrm{mmol})$ in $2 \mathrm{~mL} \mathrm{CH}_{2} \mathrm{Cl}_{2}$ was added trifluoroacetic anhydride (35 $\mu \mathrm{L}, 0.25 \mathrm{mmol}, 1.0$ equiv) at $0^{\circ} \mathrm{C}$ under nitrogen. The resulting mixture was stirred for $30 \mathrm{~min}$ at $0^{\circ} \mathrm{C}$ and was quenched by pouring the mixture into a cold, saturated $\mathrm{NaHCO}_{3}(20 \mathrm{~mL})$ solution. Aqueous layer was extracted with $\mathrm{CH}_{2} \mathrm{Cl}_{2}(3 \mathrm{X} 20 \mathrm{~mL})$. The combined organic layers were washed with water $(20 \mathrm{~mL})$, dried over $\mathrm{MgSO}_{4}$, and concentrated under reduced pressure. The product $( \pm)-42$ (43 $\mathrm{mg}, 70 \%)$ was obtained as colorless oil after bulb-to-bulb distillation of the residue.

Data for $( \pm)-\mathbf{4 2}$ :

bp: $\quad 150{ }^{\circ} \mathrm{C}$ ABT $(0.3 \mathrm{mmHg})$

${ }^{1}$ H NMR: $\quad\left(500 \mathrm{MHz}, \mathrm{CDCl}_{3}\right)$

$8.40\left(\mathrm{dd}, J=1.5,0.8,1 \mathrm{H}, \mathrm{HC}\left(6^{\prime}\right)\right), 7.49\left(\mathrm{dd}, J=7.8,1.4,1 \mathrm{H}, \mathrm{HC}\left(4^{\prime}\right)\right), 7.17$ (d, $J$ = 7.8, $1 \mathrm{H}, \mathrm{H}\left(\mathrm{C}\left(3^{\prime}\right)\right), 5.67$ (s, $\left.1 \mathrm{H}, \mathrm{HC}(1)\right), 2.34\left(\mathrm{~s}, 3 \mathrm{H}, \mathrm{H}_{3} \mathrm{C}\left(5^{\prime}\right)\right), 1.01(\mathrm{~s}, 9 \mathrm{H}$, $\left.\left(\mathrm{H}_{3} \mathrm{C}\right)_{3} \mathrm{C}(2)\right)$

GC: $\quad(R)-\mathbf{4 2}, t_{\mathrm{R}} 6.10 \min (50.1 \%) ;(S)-\mathbf{4 2}, t_{\mathrm{R}} 7.37 \min (49.8 \%)$ (Chiraldex GTA, $88^{\circ} \mathrm{C}$, $18 \mathrm{psi})$ 


\section{4. $\quad(R)-1-[2-(5-M e t h y l p y r i d y l)]-2,2-d i m e t h y l p r o p a n o l ~((R)-33)$}
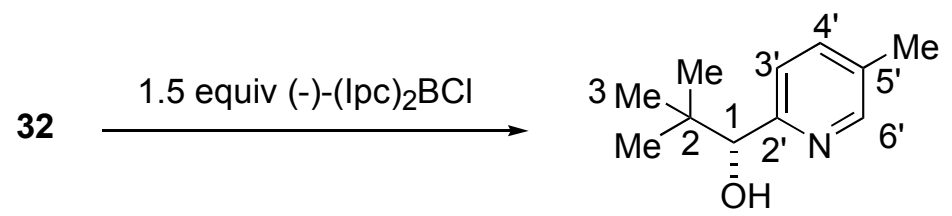

$(R)-33$

A mixture of (-)-(Ipc) $2 \mathrm{BCl}(11.8 \mathrm{~g}, 36.8 \mathrm{mmol}, 1.5$ equiv) and 32 (4.34 g, $24.5 \mathrm{mmol})$ were stirred neat at $\mathrm{rt}$. The mixture became very viscous after $2 \mathrm{~h}$ and $5 \mathrm{~mL}$ THF was added to decrease the viscosity. After stirring for 3 days at $\mathrm{rt}, \mathrm{Et}_{2} \mathrm{O}(80 \mathrm{~mL})$ was added into the flask, followed by diethanolamine (12 g). The mixture was stirred for $3 \mathrm{~h}$ at $\mathrm{rt}$ and the white precipitate was filtered off through Celite. The filtrate was dried $\left(\mathrm{Na}_{2} \mathrm{SO}_{4}\right)$ and concentrated under reduced pressure. Purification of the oily residue by silica gel column chromatography (hexane/EtOAc 5/1) afforded $3.68 \mathrm{~g}$ of product $(R)-33(84 \%)$ as colorless oil. An analytically pure sample was obtained after bulb-to-bulb distillation.

Data for $(R)-33$ :

bp: $\quad 110^{\circ} \mathrm{C} \operatorname{ABT}(0.5 \mathrm{mmHg})$

${ }^{1} \underline{\mathrm{H} N M R}: \quad\left(500 \mathrm{MHz}, \mathrm{CDCl}_{3}\right)$

8.37 (bs, $\left.1 \mathrm{H}, \mathrm{HC}\left(6^{\prime}\right)\right), 7.43\left(\mathrm{dd}, J=8.0,1.7,1 \mathrm{H}, \mathrm{HC}\left(4^{\prime}\right)\right), 7.09$ (d, $J=7.8,1 \mathrm{H}$, $\mathrm{H}\left(\mathrm{C}\left(3^{\prime}\right)\right)$, 4.24-4.33 (AB quartet, $J=7.1,2 \mathrm{H}, \mathrm{HC}(1)$ and $\left.\mathrm{OH}\right), 2.33$ (s, $3 \mathrm{H}$, $\left.\mathrm{H}_{3} \mathrm{C}\left(5^{\prime}\right)\right), 0.91\left(\mathrm{~s}, 9 \mathrm{H},\left(\mathrm{H}_{3} \mathrm{C}\right)_{3} \mathrm{C}(2)\right)$

${ }^{13} \mathrm{C}$ NMR: $\quad\left(100 \mathrm{MHz}, \mathrm{CDCl}_{3}\right)$

$156.9\left(\mathrm{C}\left(2^{\prime}\right)\right), 147.6\left(\mathrm{C}\left(6^{\prime}\right)\right), 136.6\left(\mathrm{C}\left(4^{\prime}\right)\right), 131.9\left(\mathrm{C}\left(5^{\prime}\right)\right), 122.5\left(\mathrm{C}\left(3^{\prime}\right)\right), 79.8$ $(\mathrm{C}(1)), 36.3\left(\mathrm{CH}_{3} \mathrm{C}\left(5^{\prime}\right)\right), 25.8(\mathrm{C}(3)), 18.1(\mathrm{C}(2))$

IR: $\quad(\mathrm{KBr})$

3161 (s), 2969 (s), 2848 (s), 2900 (s), 2867 (s), 2740 (m), 1606 (w), 1573 (m), 1486 (s), 1454 (s), 1429 (m), 1388 (s), 1363 (s), 1348 (m), 1307 (w), 1240 (m), 1209 (m), 1182 (w),1132 (w), 1079 (s), 1037 (s), 1018 (s), 910 (w), 837 (m), 798 (w)

TLC: $\quad R_{f} 0.20$ (hexane/EtOAc, 5/1) 
MS: $\quad(F I, 70 \mathrm{eV})$

181 (3), 180 (31), 179 (100), 123 (6), 122 (73)

Opt. Rot.: $\quad[\alpha]_{\mathrm{D}}^{24}+29.4(c=0.89, \mathrm{EtOH})$

Analysis: $\quad \mathrm{C}_{11} \mathrm{H}_{17} \mathrm{NO}(177.25)$
Calcd:
C, 73.10;
H, 9.56;
$\mathrm{N}, 7.81 \%$
Found:
C, 73.05;
H, 9.73;
$\mathrm{N}, 7.83 \%$

\section{5. $\quad(R)$-1-[2-(5-Methylpyridyl)]-2,2-dimethylpropyl Trifluoroacetate $((R)-43)$}

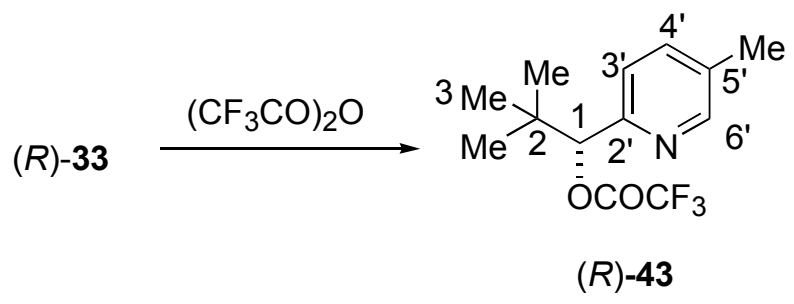

To a solution of $(R)-33(180 \mathrm{mg}, 1 \mathrm{mmol})$ in $\mathrm{CH}_{2} \mathrm{Cl}_{2}(10 \mathrm{~mL})$ was added trifluoroacetic anhydride (160 $\mu \mathrm{L}, 1.1 \mathrm{mmol}, 1.1$ equiv) at $0^{\circ} \mathrm{C}$ under $\mathrm{N}_{2}$. The resulting solution was stirred for $1 \mathrm{~h}$ at $0{ }^{\circ} \mathrm{C}$ and was quenched by pouring the mixture into a cold, saturated aqueous solution of $\mathrm{NaHCO}_{3}(20 \mathrm{~mL})$. The aqueous layer was extracted with $\mathrm{CH}_{2} \mathrm{Cl}_{2}(3 \mathrm{X} 20 \mathrm{~mL})$. The combined organic layers were washed with water $(20 \mathrm{~mL})$, dried $\left(\mathrm{MgSO}_{4}\right)$ and concentrated under reduced pressure. The product $(R)-\mathbf{4 3}(244 \mathrm{mg}, 90 \%)$ was obtained as colorless oil after bulb-to-bulb distillation.

Data for $(R)-\mathbf{4 3}$ :

bp: $\quad 150^{\circ} \mathrm{C}$ ABT $(0.3 \mathrm{mmHg})$

${ }^{1} \mathrm{H}$ NMR: $\quad\left(500 \mathrm{MHz}, \mathrm{CDCl}_{3}\right)$

$8.40\left(\mathrm{dd}, J=1.5,0.8,1 \mathrm{H}, \mathrm{HC}\left(6^{\prime}\right)\right), 7.49\left(\mathrm{dd}, J=7.8,1.4,1 \mathrm{H}, \mathrm{HC}\left(4^{\prime}\right)\right), 7.17(\mathrm{~d}, J$ $=7.8,1 \mathrm{H}, \mathrm{H}\left(\mathrm{C}\left(3^{\prime}\right)\right), 5.67(\mathrm{~s}, 1 \mathrm{H}, \mathrm{HC}(1)), 2.34\left(\mathrm{~s}, 3 \mathrm{H}, \mathrm{H}_{3} \mathrm{C}\left(5^{\prime}\right)\right), 1.01(\mathrm{~s}, 9 \mathrm{H}$, $\left.\left(\mathrm{H}_{3} \mathrm{C}\right)_{3} \mathrm{C}(2)\right)$

${ }^{13} \mathrm{C}$ NMR: $\quad\left(100 \mathrm{MHz}, \mathrm{CDCl}_{3}\right)$

$162.1(\mathrm{q}, J=35.9, \mathrm{C}(\mathrm{C}=\mathrm{O})), 152.9\left(\mathrm{C}\left(2^{\prime}\right)\right), 149.1\left(\mathrm{C}\left(6^{\prime}\right)\right), 136.9\left(\mathrm{C}\left(4^{\prime}\right)\right), 132.9$ $\left(\mathrm{C}\left(5^{\prime}\right)\right), 122.5\left(\mathrm{C}\left(3^{\prime}\right)\right), 116.6\left(\mathrm{q}, J=271.8, \mathrm{CF}_{3}\right), 87.4(\mathrm{C}(1)), 35.3\left(\mathrm{CH}_{3} \mathrm{C}\left(5^{\prime}\right)\right), 25.8$ $(\mathrm{C}(3)), 18.1(\mathrm{C}(2))$ 
IR: (neat)

2973 (s), 2974 (m), 1786 (s), 1601 (w), 1575 (m), 1486 (m), 1247 (m), 1399 (m), 1370 (s), 1337 (w), 1222 (s), 1157 (s), 1030 (m), 949 (m), 874 (w), 834 (m), 784 (m), $772(\mathrm{w}), 742(\mathrm{w}), 719(\mathrm{~m})$

TLC: $\quad \operatorname{Rf} 0.32$ (hexane/EtOAc, 10/1)

GC: $\quad(R)-43, t_{\mathrm{R}} 6.10 \min (97.8 \%) ;(S)-\mathbf{4 3}, t_{\mathrm{R}} 7.37 \min (2.2 \%)$ (Chiraldex GTA, $88{ }^{\circ} \mathrm{C}$, 18 psi)

${ }^{19}$ F NMR: $\quad\left(376 \mathrm{MHz}, \mathrm{CDCl}_{3}\right)$

$-75.55$

Analysis: $\mathrm{C}_{13} \mathrm{H}_{16} \mathrm{~F}_{3} \mathrm{NO}_{2}(275.27)$

Calcd: $\quad$ C, 56.72; $\quad \mathrm{H}, 5.86 ; \quad \mathrm{N}, 5.09 \%$

Found: $\quad$ C, 56.90; H, 5.91; N, 5.12\%

6. (R)-5-Methyl-1-(1-butyloxy-2,2-dimethylpropyl)pyridine ((R)-34)

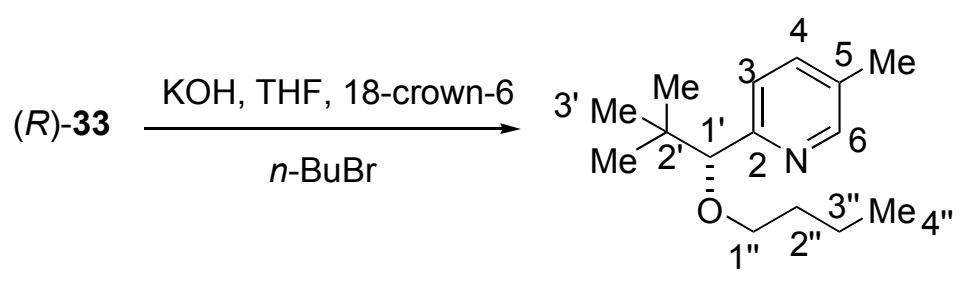

(R)-34

The mixture of $(R)-33(880 \mathrm{mg}, 4.9 \mathrm{mmol})$, potassium hydroxide (540 mg, $9.8 \mathrm{mmol}, 2.0$ equiv), $n$-BuBr (790 $\mu \mathrm{L}, 9.3 \mathrm{mmol}, 1.9$ equiv), 18-crown-6 (130 mg, $0.49 \mathrm{mmol}, 0.1 \mathrm{equiv})$ in DMF $(10 \mathrm{~mL})$ was stirred in the presence of freshly dried molecular sieve power $\left(2 \mathrm{~g}, 3 \mathrm{~A}^{\circ}\right)$ at $\mathrm{rt}$. The second and third portion of $n-\mathrm{BuBr}$ (each of $790 \mu \mathrm{L}, 1.9$ equiv), and $\mathrm{KOH}$ (each of $540 \mathrm{mg}$, 2.0 equiv) were added after $4 \mathrm{~h}$ and $8 \mathrm{~h}$, respectively. After $12 \mathrm{~h}$, the reaction mixture was diluted with $\mathrm{Et}_{2} \mathrm{O}(40 \mathrm{~mL})$ and filtered through Celite. The filtrate was dried $\left(\mathrm{MgSO}_{4}\right)$ and concentrated under reduced pressure. Purification of the residue by column silica gel chromatography (hexane/EtOAc, 5/1) afforded (R)-34 (967 mg, 84\%) as colorless oil. An analytically pure sample was obtained after bulb-to-bulb distillation. 


\section{Data for $(R)-34:$}

bp: $\quad 110^{\circ} \mathrm{C}$ ABT $(0.1 \mathrm{mmHg})$

${ }^{1} \mathrm{H} \mathrm{NMR}: \quad\left(400 \mathrm{MHz}, \mathrm{CDCl}_{3}\right)$

$8.36(\mathrm{dd}, J=1.5,0.7,1 \mathrm{H}, \mathrm{HC}(6)), 7.24(\mathrm{dd}, J=8.0,1.2,1 \mathrm{H}, \mathrm{HC}(4)), 7.26$ (d, $J=$ 8.1, 1 H, H(C(3)), 4.00 (s, $\left.1 \mathrm{H}, \mathrm{HC}\left(1^{\prime}\right)\right)$ ) 3.19-3.31 (m, 2 H, $\left.\mathrm{H}_{2} \mathrm{C}\left(1^{\prime \prime}\right)\right), 2.32$ (s, $3 \mathrm{H}$, $\left.\mathrm{H}_{3} \mathrm{C}(\mathrm{C}(5))\right)$, 1.41-1.55 (m, $\left.2 \mathrm{H}, \mathrm{H}_{2} \mathrm{C}\left(2^{\prime \prime}\right)\right)$, 1.32-1.40 (m, $2 \mathrm{H}, \mathrm{H}_{2} \mathrm{C}\left(3^{\prime \prime}\right)$ ), 0.90 (s, 9 $\left.\mathrm{H},\left(\mathrm{H}_{3} \mathrm{C}\right)_{3}\left(\mathrm{C}\left(3^{\prime}\right)\right)\right), 0.88\left(\mathrm{t}, J=7.3,3 \mathrm{H}, \mathrm{H}_{3} \mathrm{C}\left(4^{\prime \prime}\right)\right)$

${ }^{13} \mathrm{C} \mathrm{NMR}: \quad\left(100 \mathrm{MHz}, \mathrm{CDCl}_{3}\right)$

$158.2(\mathrm{C}(2)), 148.4(\mathrm{C}(6)), 136.3(\mathrm{C}(4)), 131.2(\mathrm{C}(5)), 121.6(\mathrm{C}(3)), 90.6\left(\mathrm{C}\left(1^{\prime}\right)\right)$, $69.4\left(\mathrm{C}\left(1^{\prime \prime}\right)\right), 35.6\left(\mathrm{CH}_{3}(\mathrm{C}(5)), 32.0\left(\mathrm{C}\left(2^{\prime \prime}\right)\right), 26.2\left(\mathrm{C}\left(3^{\prime}\right)\right), 19.4\left(\mathrm{C}\left(3^{\prime \prime}\right)\right), 18.1\right.$ $\left(\mathrm{C}\left(2^{\prime}\right)\right), 13.9\left(\mathrm{C}\left(4^{\prime \prime}\right)\right)$

IR: (neat)

2957 (s), 2932 (s), 2869 (s), $1601(w), 1569$ (w),1482 (m), 1242 (w), 1390 (w), $1100(\mathrm{~m}), 1030(\mathrm{w}), 858(\mathrm{w}), 836(\mathrm{w})$

TLC: $\quad R_{f} 0.24$ (hexane/EtOAc, 5/1)

MS: (EI)

234 (6), 179 (18), 178 (33), 122 (100), 92 (11)

Opt. Rot: $\quad[\alpha]_{\mathrm{D}}^{24}+37.8(c=0.67, \mathrm{EtOH})$

Analysis: $\mathrm{C}_{15} \mathrm{H}_{25} \mathrm{NO}$ (235.37)

Calcd: $\quad$ C, 76.54; H, 10.71; N, 5.95\%

Found: $\quad$ C, $76.45 ; \quad \mathrm{H}, 10.89 ; \quad \mathrm{N}, 6.11 \%$ 
7. (R)-5-Methyl-1-(1-butyloxy-2,2-dimethylpropyl)pyridine $N$-Oxide $((R)-35)$

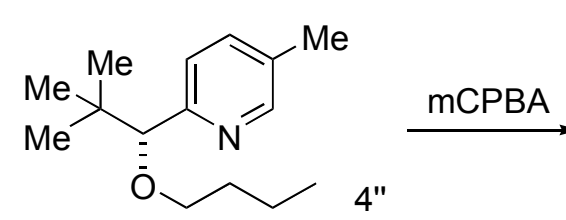

(R)-34

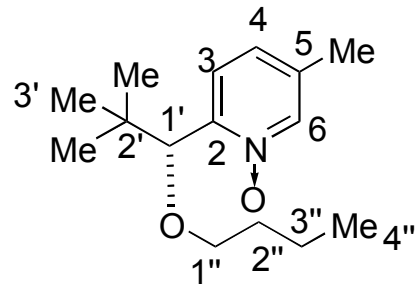

$(R)-35$

Following General Procedure III, pyridine $(R)-34(1.67 \mathrm{~g}, 7.0 \mathrm{mmol})$ and $m \mathrm{CPBA}(1.84$ g, 10.6 mmol, 1.5 equiv) were dissolved in $\mathrm{CH}_{2} \mathrm{Cl}_{2}(25 \mathrm{~mL})$ and the mixture was stirred overnight at rt. Work-up provided $1.82 \mathrm{~g}$ of white solid residue. Purification by silica gel column chromatography $\left(\mathrm{CH}_{2} \mathrm{Cl}_{2} / \mathrm{MeOH}, 20 / 1\right)$ provided the product $(1.49 \mathrm{~g}, 84 \%)$ as colorless oil. An analytically pure sample was obtained after bulb-to-bulb distillation.

Data for $(R)-35$ :

bp: $\quad 150{ }^{\circ} \mathrm{C}$ ABT $(0.1 \mathrm{mmHg})$

${ }^{1} \mathrm{H}$ NMR: $\quad\left(400 \mathrm{MHz}, \mathrm{CDCl}_{3}\right)$

8.06 (s, $1 \mathrm{H}, \mathrm{HC}(6)), 7.29$ (d, J = 8.3, $1 \mathrm{H}, \mathrm{HC}(3)), 7.05$ (d, J = 8.0, $1 \mathrm{H}, \mathrm{H}(\mathrm{C}(4))$, 5.02 (s, $\left.1 \mathrm{H}, \mathrm{HC}\left(1^{\prime}\right)\right)$, 3.15-3.23 (m, $2 \mathrm{H}, \mathrm{H}_{2} \mathrm{C}\left(1^{\prime \prime}\right)$ ), 2.23 (s, $3 \mathrm{H}, \mathrm{H}_{3} \mathrm{C}(\mathrm{C}(5))$ ), 1.341.49 (m, $\left.2 \mathrm{H}, \mathrm{H}_{2} \mathrm{C}\left(2^{\prime \prime}\right)\right)$, 1.23-1.39 (m, $\left.2 \mathrm{H}, \mathrm{H}_{2} \mathrm{C}\left(3^{\prime \prime}\right)\right), 0.96\left(\mathrm{~s}, 9 \mathrm{H},\left(\mathrm{H}_{3} \mathrm{C}\right)_{3}\left(\mathrm{C}\left(3^{\prime}\right)\right)\right.$, $0.86\left(\mathrm{t}, J=7.3,3 \mathrm{H}, \mathrm{H}_{3} \mathrm{C}\left(4^{\prime \prime}\right)\right)$

${ }^{13} \mathrm{C}$ NMR: $\quad\left(100 \mathrm{MHz}, \mathrm{CDCl}_{3}\right)$

$149.3(\mathrm{C}(2)), 139.5(\mathrm{C}(6)), 134.4(\mathrm{C}(5)), 126.3(\mathrm{C}(3)$ or $\mathrm{C}(4)), 125.1$ (C(3) or $\mathrm{C}(4)), 80.2$ (C(1')), $69.9\left(\mathrm{C}\left(1^{\prime \prime}\right)\right), 37.2\left(\mathrm{CH}_{3}(\mathrm{C}(5)), 31.9\left(\mathrm{C}\left(2^{\prime \prime}\right)\right), 25.6\left(\mathrm{C}\left(3^{\prime}\right)\right), 19.4\right.$ $\left(\mathrm{C}\left(3^{\prime \prime}\right)\right), 18.0\left(\mathrm{C}\left(2^{\prime}\right)\right), 13.9\left(\mathrm{C}\left(4^{\prime \prime}\right)\right)$

IR: (neat)

2957 (s), 2871 (m), 1613 (w), 1503 (w), 1478 (w), 1241 (w), 1383 (m), 1363 (m), 1339 (m), 1295 (m), 1256 (m), 1213 (m), 1199 (m), 1177 (s), 1095 (s), 1020 (w)

TLC: $\quad R_{f} 0.42\left(\mathrm{CH}_{2} \mathrm{Cl}_{2} / \mathrm{MeOH}, 20 / 1\right)$

MS: (FAB) 253 (17), 252 (100), 296 (18), 195 (12), 178 (18), 119 (17) 
Opt. Rot.: $[\alpha]_{\mathrm{D}}^{24}+87.5(c=0.535, \mathrm{EtOH})$

Analysis: $\quad \mathrm{C}_{15} \mathrm{H}_{25} \mathrm{NO}_{2}(251.37)$

$\begin{array}{llll}\text { Calcd: } & \text { C }, 71.67 ; & \text { H, 10.02; } & \text { N, 5.57\% } \\ \text { Found: } & \text { C, 71.55; } & \text { H, 10.10; } & \text { N, 5.76\% }\end{array}$

8. $\quad(P)-(R, R)-3,3$ '-Dimethyl-6,6'-bis-(1-butyloxy-2,2-dimethylpropyl))-2,2'-bipyridine Bis- $N$-oxide $((P)-(R, R)-19)$

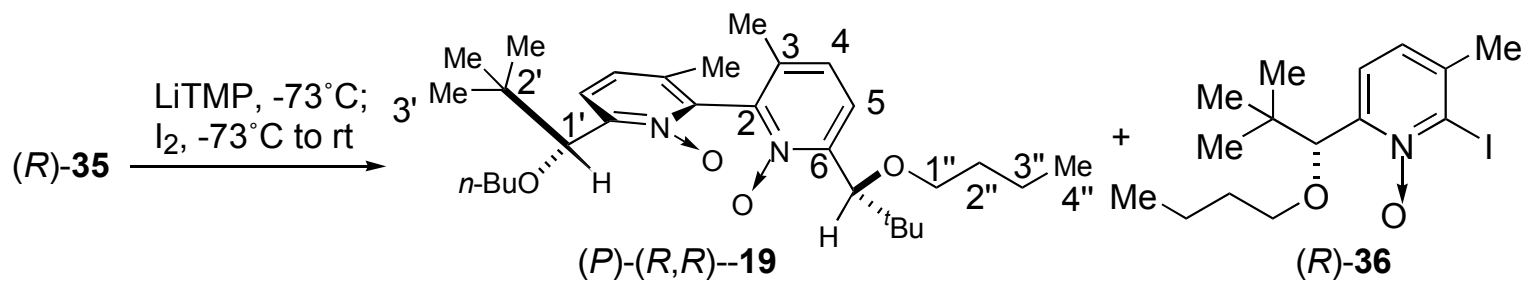

To a suspension of $(R)-35(2.51 \mathrm{~g}, 10 \mathrm{mmol})$ in $\mathrm{Et}_{2} \mathrm{O}(20 \mathrm{~mL})$ was added freshly prepared LiTMP solution $(n$-BuLi, $6.5 \mathrm{~mL}, 10 \mathrm{mmol}, 1.0$ equiv, and tetramethylpiperidine $1.70 \mathrm{~mL}, 10$ mmol, 1.0 equiv $)$ in THF $(10 \mathrm{~mL})$ at $-73{ }^{\circ} \mathrm{C}$. After being stirred for $16 \mathrm{~h}$ at $-73{ }^{\circ} \mathrm{C}$, a solution of iodine $(1.17 \mathrm{~g}, 5.0 \mathrm{mmol}, 0.5$ equiv) in THF $(10 \mathrm{~mL})$ was added dropwise over $10 \mathrm{~min}$ into the resulting deep-red solution at $-73{ }^{\circ} \mathrm{C}$. The mixture was further stirred at $-73{ }^{\circ} \mathrm{C}$ for $1 \mathrm{~h}$ until the color of the mixture was discharged and a second portion of iodine (1.17 g, $5.0 \mathrm{mmol}, 0.5$ equiv) in THF $(10 \mathrm{~mL})$ was added into the flask. The cooling bath was immediately removed and the reaction mixture was brought up to $\mathrm{rt}$ over $40 \mathrm{~min}$. During the warming period, the deep-red color was recharged. After being stirred at $\mathrm{rt}$ for $2 \mathrm{~h}$, cold water $(10 \mathrm{~mL})$ was added into the flask, followed by sat. aq. $\mathrm{NaHSO}_{3}$ solution $(10 \mathrm{~mL})$. The mixture was transferred to a separatory funnel, diluted with $\mathrm{NH}_{4} \mathrm{OH}$ solution $(50 \mathrm{~mL})$, and was thoroughly extracted with $\mathrm{CH}_{2} \mathrm{Cl}_{2}(3 \times 60 \mathrm{~mL})$. The combined organic layers were dried $\left(\mathrm{MgSO}_{4}\right)$ and concentrated under reduced pressure. The brownish residue was purified by silica gel column chromatography (pentane/ether, 2/1) to provide $3.0 \mathrm{~g}$ of a mixture of $(P)-(R, R)-\mathbf{1 9}$ and the by-product iodide $(R)$-36. The iodide $(1.70 \mathrm{~g}, 47 \%)$ was removed by recrystallization from pentane. From the mother liquor, $(P)-(R, R)-19(1.20 \mathrm{~g}, 48 \%)$ was obtained. An analytically pure sample was obtained after recrystallization from hexane. 
Data for $(P)-(R, R)-\mathbf{1 9}$ :

mp: $\quad 135-135.5^{\circ} \mathrm{C}$ (hexane)

${ }^{1} \mathrm{H} \mathrm{NMR}: \quad\left(400 \mathrm{MHz}, \mathrm{CDCl}_{3}\right)$

7.39 (d, $J=8.0,2$ H, HC(5)), 7.16 (d, J=8.1, 2 H, HC(4)), 5.06 (s, 2 H, HC(1')), 3.24-3.32 (m, $\left.4 \mathrm{H}, \mathrm{H}_{2} \mathrm{C}\left(1^{\prime \prime}\right)\right), 2.04\left(\mathrm{~s}, 6 \mathrm{H}, \mathrm{H}_{3} \mathrm{C}(\mathrm{C}(3))\right.$, 1.44-1.51 (m, $4 \mathrm{H}$, $\left.\mathrm{H}_{2} \mathrm{C}\left(2^{\prime \prime}\right)\right), 1.26-1.40\left(\mathrm{~m}, 4 \mathrm{H}, \mathrm{H}_{2} \mathrm{C}\left(3^{\prime \prime}\right)\right), 0.97\left(\mathrm{~s}, 18 \mathrm{H},\left(\mathrm{H}_{3} \mathrm{C}\right)_{3}\left(\mathrm{C}\left(3^{\prime}\right)\right), 0.88(\mathrm{t}, J=\right.$ 7.3, $\left.6 \mathrm{H}, \mathrm{H}_{3} \mathrm{C}\left(4^{\prime \prime}\right)\right)$

${ }^{13} \mathrm{C} \mathrm{NMR}: \quad\left(100 \mathrm{MHz}, \mathrm{CDCl}_{3}\right)$

$149.5(\mathrm{C}(6)), 142.9(\mathrm{C}(2)), 134.6(\mathrm{C}(3)), 125.9(\mathrm{C}(4)$ or $\mathrm{C}(5)), 124.8(\mathrm{C}(5)$ or $\mathrm{C}(4)), 80.3\left(\mathrm{C}\left(1^{\prime}\right)\right), 70.0\left(\mathrm{C}\left(1^{\prime \prime}\right)\right), 37.2\left(\mathrm{CH}_{3}(\mathrm{C}(3)), 32.1\left(\mathrm{C}\left(2^{\prime \prime}\right)\right), 25.7\left(\mathrm{C}\left(3^{\prime}\right)\right), 19.4\right.$ $\left(\mathrm{C}\left(3^{\prime \prime}\right)\right), 17.5\left(\mathrm{C}\left(2^{\prime}\right)\right), 14.0\left(\mathrm{C}\left(4^{\prime \prime}\right)\right)$

IR: $(\mathrm{KBr})$

2957 (s), 2871 (m), 1613 (w), 1503 (w), 1478 (w), 1241 (w), 1383 (m), 1295 (m), $1256(\mathrm{w}), 1177(\mathrm{~m}), 1095(\mathrm{~m})$

TLC: $\quad R_{f} 0.32$ (hexane/EtOAc, 5/1)

MS: (FAB)

502 (34), 501 (100), 485 (22)

Opt. Rot.: $[\alpha]_{\mathrm{D}}^{24}-39.7(c=1.03, \mathrm{EtOH})$

Analysis: $\quad \mathrm{C}_{30} \mathrm{H}_{48} \mathrm{~N}_{2} \mathrm{O}_{4}(500.72)$

Calcd: $\quad$ C, 71.96; H, 9.66; N, 5.59\%

Found: $\quad$ C, $72.01 ; \quad H, 9.90 ; \quad$ N, $5.64 \%$ 
Preparation of $(P)-(R, R)-3,3$ '-Bismethyl-6,6'-bis(1-butyloxy-2,2-dimethylpropyl))-2,2'bipyridine Bis- $N$-oxide $((M)-(R, R)-19)$

1. $\quad(R, R)-3,3$ '-Bismethyl-6,6'-bis(1-butyloxy-2,2-dimethylpropyl))-2,2'-bipyridine $((R, R)-37)$

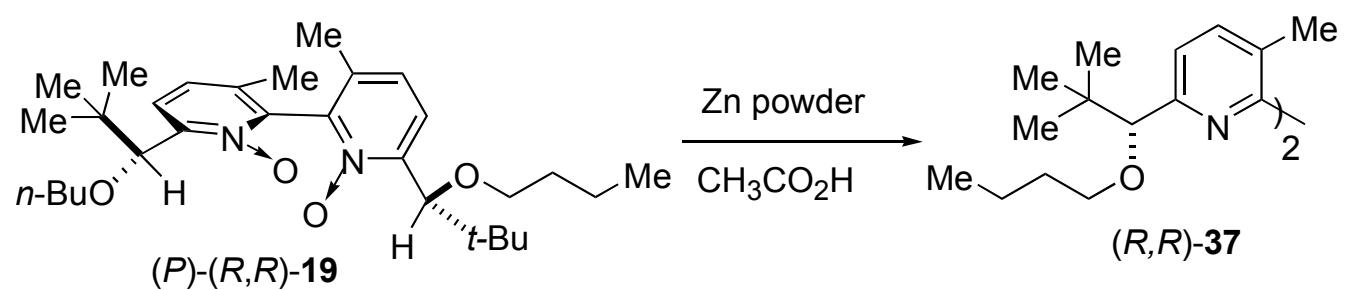

The mixture of $(P)-(R, R)-\mathbf{1 9}(1.43 \mathrm{~g}, 2.85 \mathrm{mmol})$, Zn powder $(1.86 \mathrm{~g}, 29.5 \mathrm{mmol}, 10$ equiv), and $\mathrm{CH}_{3} \mathrm{CO}_{2} \mathrm{H}$ ( 2 drop) in wet THF ( $20 \mathrm{~mL}$ ) was stirred for $12 \mathrm{~h}$ at rt. Aqueous $\mathrm{NH}_{4} \mathrm{OH}$ solution $(50 \mathrm{~mL})$ was added into the flask and stirred for $30 \mathrm{~min}$. The resulting precipitate was filtered off through Celite. The filtrate was thoroughly extracted with $\mathrm{CH}_{2} \mathrm{Cl}_{2}(3 \mathrm{X} 30 \mathrm{~mL})$. The combined organic layers were dried $\left(\mathrm{MgSO}_{4}\right)$ and concentrated under reduced pressure. The brownish residue was purified by silica gel column chromatography (hexane/EtOAc, 20/1) to provide $1.22 \mathrm{~g}(91 \%)$ of the product $(R, R)-37$ as a light yellow oil.

\section{Data for $(R, R)-37$ :}

${ }^{1}$ H NMR: $\quad\left(400 \mathrm{MHz}, \mathrm{CDCl}_{3}\right)$

7.59 (d, $J=7.8,2 \mathrm{H}, \mathrm{HC}(5)), 7.35$ (d, $J=8.0,2 \mathrm{H}, \mathrm{HC}(4)), 4.11$ (s, $\left.2 \mathrm{H}, \mathrm{HC}\left(1^{\prime}\right)\right)$, 3.18-3.38 (m, $\left.4 \mathrm{H}, \mathrm{H}_{2} \mathrm{C}\left(1^{\prime}\right)\right), 2.13\left(\mathrm{~s}, 6 \mathrm{H}, \mathrm{H}_{3} \mathrm{C}(\mathrm{C}(3))\right.$, 1.47-1.54 (m, $4 \mathrm{H}$, $\left.\mathrm{H}_{2} \mathrm{C}\left(2^{\prime \prime}\right)\right), 1.32-1.39\left(\mathrm{~m}, 4 \mathrm{H}, \mathrm{H}_{2} \mathrm{C}\left(3^{\prime \prime}\right)\right), 0.94\left(\mathrm{~s}, 18 \mathrm{H},\left(\mathrm{H}_{3} \mathrm{C}\right)_{3}\left(\mathrm{C}\left(3^{\prime}\right)\right), 0.88\right.$ (t, $J=$ 7.3, $\left.6 \mathrm{H}, \mathrm{H}_{3} \mathrm{C}\left(4^{\prime \prime}\right)\right)$

${ }^{13} \mathrm{C}$ NMR: $\quad\left(100 \mathrm{MHz}, \mathrm{CDCl}_{3}\right)$

$158.1(\mathrm{C}(6)), 155.7(\mathrm{C}(2)), 138.6(\mathrm{C}(4)), 130.2(\mathrm{C}(3)), 121.3(\mathrm{C}(5)), 90.2\left(\mathrm{C}\left(1^{\prime}\right)\right)$, $69.2\left(\mathrm{C}\left(1^{\prime \prime}\right)\right), 35.7\left(\mathrm{CH}_{3}(\mathrm{C}(3)), 32.1\left(\mathrm{C}\left(2^{\prime \prime}\right)\right), 26.2\left(\mathrm{C}\left(3^{\prime}\right)\right), 19.4\left(\mathrm{C}\left(4 \mathrm{t} 3^{\prime \prime}\right)\right), 18.8\right.$ $\left(\mathrm{C}\left(2^{\prime}\right)\right), 14.0\left(\mathrm{C}\left(4^{\prime \prime}\right)\right)$

IR: (neat)

2957 (s), 2868 (s), 1584 (s), 1569 (s), 1479 (s), 1456 (s), 1390 (s), 1364 (s), 1303 (m), 1265 (m), 1244 (m), 1207 (m), 1101 (s), 1067 (m), 1036 (m), 994 (m), 970 (m), $841(\mathrm{~m}), 737(\mathrm{~m})$ 
TLC: $\quad R_{f} 0.37$ (hexane/EtOAc, 20/1)

MS: (EI, 70eV)

249 (14), 248 (39), 413 (11), 412 (40), 411 (26), 356 (27), 355 (98), 292 (21), 231

(100), 265 (11), 241 (33)

\section{2. (M)-(R,R)-3,3'-Dimethyl-6,6'-bis-(1-butyloxy-2,2-dimethylpropyl))-2,2'-bipyridine}

Bis- $N$-oxide $((M)-(R, R)-19)$

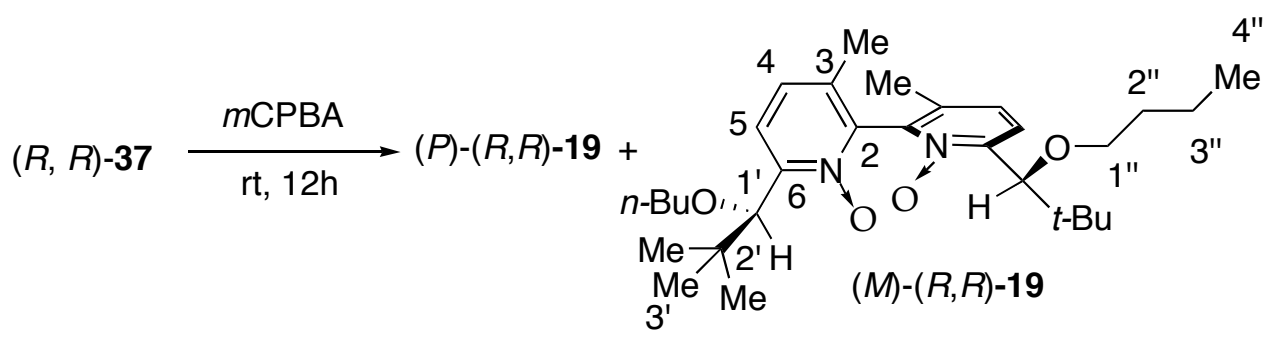

Following General Procedure III, pyridine $(R, R)-37(840 \mathrm{mg}, 1.8 \mathrm{mmol})$ and $m$ CPBA (864 mg, $5.0 \mathrm{mmol}, 2.8$ equiv) were dissolved in $\mathrm{CH}_{2} \mathrm{Cl}_{2}(20 \mathrm{~mL})$ and the mixture was stirred for $12 \mathrm{~h}$ at $\mathrm{rt}$. Work-up provided $1.11 \mathrm{~g}$ of white solid residue. Separation of the two diastereomer by silica gel column chromatography (hexane/EtOAc, 5/1) provided $601 \mathrm{mg}(67 \%)$ of $(P)-(R, R)-19$ and $242 \mathrm{mg}(27 \%)$ of $(M)-(R, R)-19$. An analytically pure sample was obtained after recrystallization from hexane.

Data for $(M)-(R, R)-19$ :

mp: $\quad 141-141.5^{\circ} \mathrm{C}$ (hexane)

${ }^{1} \underline{\mathrm{H} N M R}: \quad\left(400 \mathrm{MHz}, \mathrm{CDCl}_{3}\right)$

7.39 (d, $J=8.0,2 \mathrm{H}, \mathrm{HC}(5)), 7.16$ (d, $J=8.1,2 \mathrm{H}, \mathrm{HC}(4)), 5.06$ (s, $\left.2 \mathrm{H}, \mathrm{HC}\left(1^{\prime}\right)\right)$, 3.24-3.32 (m, $\left.4 \mathrm{H}, \mathrm{H}_{2} \mathrm{C}(1 ")\right), 2.13\left(\mathrm{~s}, 6 \mathrm{H}, \mathrm{H}_{3} \mathrm{C}(\mathrm{C}(3))\right.$, 1.44-1.51 (m, $4 \mathrm{H}$, $\left.\mathrm{H}_{2} \mathrm{C}\left(2^{\prime \prime}\right)\right), 1.26-1.40\left(\mathrm{~m}, 4 \mathrm{H}, \mathrm{H}_{2} \mathrm{C}\left(3^{\prime \prime}\right)\right), 0.97\left(\mathrm{~s}, 18 \mathrm{H},\left(\mathrm{H}_{3} \mathrm{C}\right)_{3}\left(\mathrm{C}\left(3^{\prime}\right)\right), 0.88(\mathrm{t}, J=\right.$ 7.3, $\left.6 \mathrm{H}, \mathrm{H}_{3} \mathrm{C}\left(4^{\prime \prime}\right)\right)$

${ }^{13} \mathrm{C}$ NMR: $\quad\left(100 \mathrm{MHz}, \mathrm{CDCl}_{3}\right)$

$149.5(\mathrm{C}(6)), 142.9(\mathrm{C}(2)), 134.6(\mathrm{C}(3)), 125.9(\mathrm{C}(4)$ or $\mathrm{C}(5)), 124.8(\mathrm{C}(5)$ or $\mathrm{C}(4)), 80.3\left(\mathrm{C}\left(1^{\prime}\right)\right), 70.0\left(\mathrm{C}\left(1^{\prime \prime}\right)\right), 37.2\left(\mathrm{CH}_{3}(\mathrm{C}(3)), 32.1\left(\mathrm{C}\left(2^{\prime \prime}\right)\right), 25.7\left(\mathrm{C}\left(3^{\prime}\right)\right), 19.4\right.$ $\left(\mathrm{C}\left(3^{\prime \prime}\right)\right), 17.5\left(\mathrm{C}\left(2^{\prime}\right)\right), 14.0\left(\mathrm{C}\left(4^{\prime \prime}\right)\right)$ 
IR: $\quad(\mathrm{KBr})$

2957 (s), 2871 (m), 1613 (w), 1503 (w), 1478 (w), 1241 (w), 1383 (m), 1295 (m), $1256(\mathrm{w}), 1177(\mathrm{~m}), 1095(\mathrm{~m})$

TLC: $\quad R_{f} 0.29$ (hexane/EtOAc, 5/1)

MS: (FAB)

502 (33), 501 (100), 485 (25)

Opt. Rot.: $[\alpha]_{\mathrm{D}}^{24}+125.9(c=0.74, \mathrm{EtOH})$

Analysis: $\quad \mathrm{C}_{30} \mathrm{H}_{48} \mathrm{~N}_{2} \mathrm{O}_{4}(500.72)$

$\begin{array}{llll}\text { Calcd: } & \text { C , 71.96; } & \text { H, 9.66; } & \text { N }, 5.59 \% \\ \text { Found: } & \text { C , 72.03; } & \text { H, 9.88; } & \text { N }, 5.66 \%\end{array}$

General Procedure IV. VT NMR Rate Studies of N-Oxide Promoted Aldol Additions of Methyl Trichlorosilyl Ketene Acetal To Acetophenone

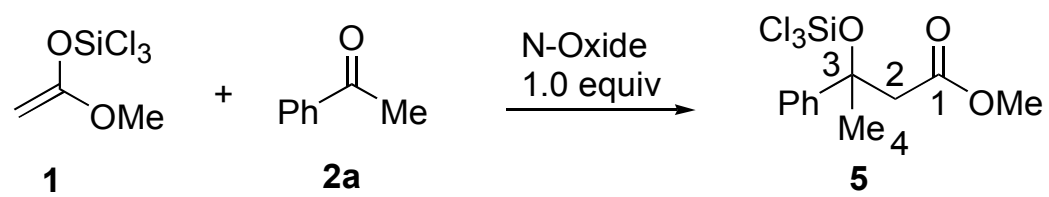

Methyl trichlorosilyl ketene acetal $1(20 \mu \mathrm{L}, \mathrm{d}=1.3144,0.12 \mathrm{mmol}, 1.2$ equiv) was added to a mixture of acetophenone $\mathbf{2 a}(12 \mu \mathrm{L}, \mathrm{d}=1.02,0.10 \mathrm{mmol}, 1.0$ equiv), N-Oxide (0.10 mmol, 1.0 equiv) in $\mathrm{CD}_{2} \mathrm{Cl}_{2}(0.7 \mathrm{~mL}$, dried over molecular sieves $)$ at $-78{ }^{\circ} \mathrm{C}$ under Argon in a flamed dried NMR tube with a septum. The tube was shaken on a Vortex shaker for 10 seconds and immediately returned to the cooling bath. The reaction was followed by $500 \mathrm{MHz}$ VTNMR.

Data for 5:

${ }^{1} \mathrm{H}$ NMR: $\quad\left(500 \mathrm{MHz}, \mathrm{CD}_{2} \mathrm{Cl}_{2}\right)$

7.17-7.28 (m, $5 \mathrm{H}$, Aryl); 3.55 (s, $\left.3 \mathrm{H}, \mathrm{OCH}_{3}\right)$; 2.93 (AB quartet, $J=15.9,2 \mathrm{H}$, $\left.\mathrm{H}_{2} \mathrm{C}(2)\right) ; 2.01$ (s, $\left.3 \mathrm{H}, \mathrm{H}_{3} \mathrm{C}(4)\right)$ 
VT NMR Studies of $(P)-(R, R)-19 \cdot \mathrm{SiCl}_{4}$ Complex

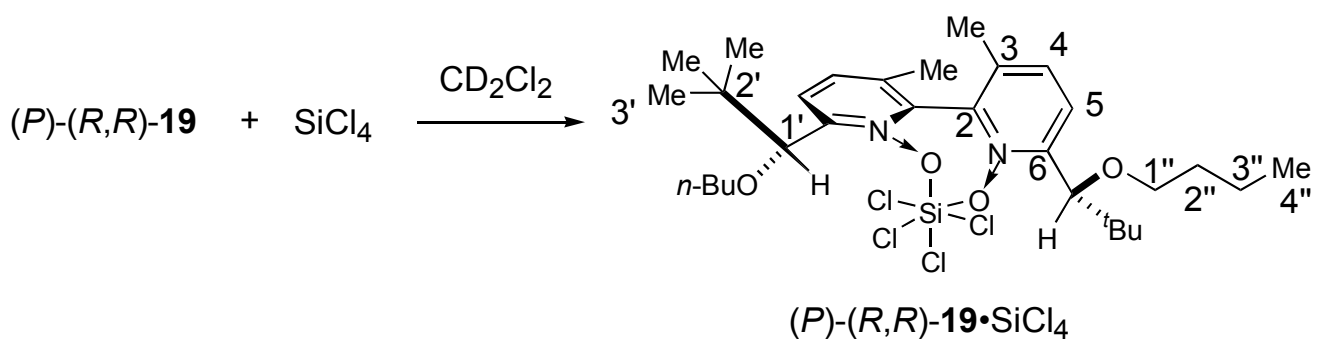

Silicon tetrachloride ( $6 \mu \mathrm{L}, \mathrm{d}=1.483,0.05 \mathrm{mmol}, 1.0$ equiv) was added to a solution of $(P)-(R, R)-19$ (25 mg, $0.05 \mathrm{mmol}, 1.0$ equiv) in $\mathrm{CD}_{2} \mathrm{Cl}_{2}(0.7 \mathrm{~mL}$, dried over molecular sieves) at $-78{ }^{\circ} \mathrm{C}$ under Argon in a flamed dried NMR tube with a septum. The tube was shaken on a Vortex shaker for 10 seconds and immediately returned to the cooling bath. The reaction was followed by $500 \mathrm{MHz}$ VT-NMR.

Data for $(P)-(R, R)-\mathbf{1 9} \cdot \mathrm{SiCl}_{4}$ :

${ }^{1} \mathrm{H} \mathrm{NMR}: \quad\left(500 \mathrm{MHz}, \mathrm{CD}_{2} \mathrm{Cl}_{2}\right.$ at $\left.-78^{\circ} \mathrm{C}\right)$

$8.12(\mathrm{~d}, J=8.0,2 \mathrm{H}, \mathrm{HC}(5)), 7.97$ (d, $J=8.1,2 \mathrm{H}, \mathrm{HC}(4)), 5.62$ (s, $\left.2 \mathrm{H}, \mathrm{HC}\left(1^{\prime}\right)\right)$, 3.51-3.75 (m, $\left.4 \mathrm{H}, \mathrm{H}_{2} \mathrm{C}\left(1^{\prime \prime}\right)\right), 2.30$ (s, $6 \mathrm{H}, \mathrm{H}_{3} \mathrm{C}(\mathrm{C}(3))$, 1.44-1.51 (m, $4 \mathrm{H}$, $\left.\mathrm{H}_{2} \mathrm{C}\left(2^{\prime \prime}\right)\right), 1.26-1.40\left(\mathrm{~m}, 4 \mathrm{H}, \mathrm{H}_{2} \mathrm{C}\left(3^{\prime \prime}\right)\right), 0.97$ (s, $18 \mathrm{H},\left(\mathrm{H}_{3} \mathrm{C}\right)_{3}\left(\mathrm{C}\left(3^{\prime}\right)\right), 0.88(\mathrm{t}, J=$ 7.3, $\left.6 \mathrm{H}, \mathrm{H}_{3} \mathrm{C}\left(4^{\prime \prime}\right)\right)$

${ }^{29}$ Si NMR: $\quad\left(100 \mathrm{MHz}, \mathrm{CD}_{2} \mathrm{Cl}_{2}\right.$ at $\left.-20{ }^{\circ} \mathrm{C}\right)$ $-167$ 
VT NMR Studies of 1/1 mixture of $(P)-(R, R)-19$ and 1

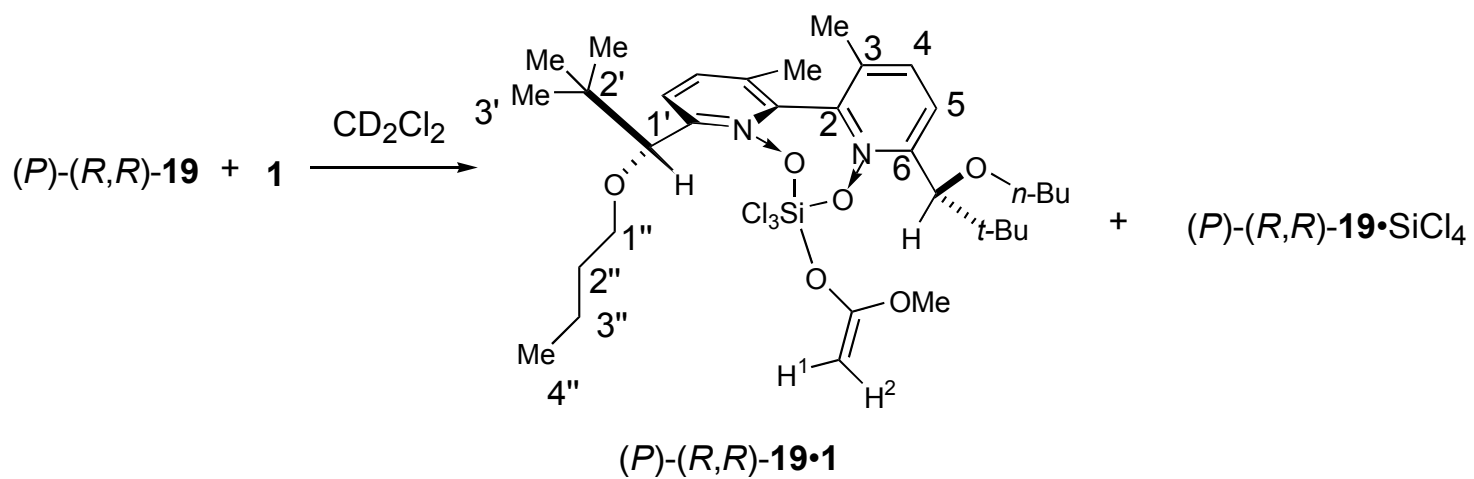

Methyl trichlorosilyl ketene acetal $1(10 \mu \mathrm{L}, \mathrm{d}=1.3144,0.06 \mathrm{mmol}, 1.0$ equiv) was added to a solution of $(P)-(R, R)-\mathbf{1 9}\left(30 \mathrm{mg}, 0.06 \mathrm{mmol}, 1.0\right.$ equiv) in $\mathrm{CD}_{2} \mathrm{Cl}_{2}(0.7 \mathrm{~mL}$, dried over molecular sieves) at $-78{ }^{\circ} \mathrm{C}$ under Argon in a flamed dried NMR tube with a septum. The tube was shaken on a Vortex shaker for 10 seconds and immediately returned to the cooling bath. The reaction was followed by $500 \mathrm{MHz}$ VT-NMR. By comparison of the ${ }^{1} \mathrm{H}$ NMR and ${ }^{29} \mathrm{Si}$ NMR spectra of this mixture with those of the 1/1 mixture of $(P)-(R, R)-\mathbf{1 9}$ and $\mathrm{SiCl}_{4}$, two complexes $(P)-(R, R)-\mathbf{1 9 \bullet 1}$ and $(P)-(R, R)-\mathbf{1 9} \cdot \mathrm{SiCl}_{4}$ were identified. The ratio of $(P)-(R, R)-\mathbf{1 9 \bullet 1}$ to $(P)-(R, R)-19 \cdot \mathrm{SiCl}_{4}$ was calculated based on the integrated intensity of the corresponding benzylic proton $\left(\mathrm{HC}\left(1^{\prime}\right)\right)$.

Data for $(P)-(R, R)-\mathbf{1 9 \bullet 1}$ :

${ }^{1} \mathrm{H} \mathrm{NMR}: \quad\left(500 \mathrm{MHz}, \mathrm{CD}_{2} \mathrm{Cl}_{2}\right.$ at $\left.-78^{\circ} \mathrm{C}\right)$

8.05 (d, $J=8.0,2$ H, HC(5)), 8.03 (d, $J=8.1,2$ H, HC(4)), 5.82 (s, $\left.1 \mathrm{H}, \mathrm{HC}\left(1^{\prime}\right)\right)$, 5.61 (s, $\left.1 \mathrm{H}, \mathrm{HC}\left(1^{\prime}\right)\right)$ ) 3.32-3.55 (m, $\left.4 \mathrm{H}, \mathrm{H}_{2} \mathrm{C}\left(1^{\prime \prime}\right)\right)$ ), 3.18 (bs, $\left.1 \mathrm{H}, \mathrm{HC}\left(5^{\prime}\right)\right), 2.95$ (bs, $\left.3 \mathrm{H}, \mathrm{H}_{3} \mathrm{C}\left(6^{\prime}\right)\right)$ ), 2.87 (bs, $\left.1 \mathrm{H}, \mathrm{H}_{3} \mathrm{C}\left(7^{\prime}\right)\right)$ ), 2.30 (s, $6 \mathrm{H}, \mathrm{H}_{3} \mathrm{C}(\mathrm{C}(3))$ ) 1.44-1.51 (m, $\left.4 \mathrm{H}, \mathrm{H}_{2} \mathrm{C}\left(2^{\prime \prime}\right)\right)$, 1.26-1.40 (m, $\left.4 \mathrm{H}, \mathrm{H}_{2} \mathrm{C}\left(3^{\prime \prime}\right)\right), 0.97$ (s, $18 \mathrm{H},\left(\mathrm{H}_{3} \mathrm{C}\right)_{3}\left(\mathrm{C}\left(3^{\prime}\right)\right), 0.88$ (t, $\left.J=7.3,6 \mathrm{H}, \mathrm{H}_{3} \mathrm{C}\left(4^{\prime \prime}\right)\right)$

${ }^{29}$ Si NMR: $\quad\left(100 \mathrm{MHz}, \mathrm{CD}_{2} \mathrm{Cl}_{2}\right.$ at $\left.-20{ }^{\circ} \mathrm{C}\right)$ $-163$ 


\section{Crystal Preparation for $(P)-(R, R)-19 \cdot \mathrm{SiCl}_{4}$}

A. The apparatus for the preparation: an oven-dried vial $(\varnothing 12 \mathrm{~mm} \mathrm{X} \mathrm{15mm)} \mathrm{is} \mathrm{placed}$ inside a flamed-dried vesicle $(\varnothing 25 \mathrm{~mm}$ X 50mm) with a septum and a nitrogen inlet.

B. A solution of $(P)-(R, R)-19(50 \mathrm{mg}, 0.1 \mathrm{mmol})$ in $\mathrm{CH}_{2} \mathrm{Cl}_{2}(0.5 \mathrm{~mL})$ was prepared inside the small vial under nitrogen and was cooled to $-78{ }^{\circ} \mathrm{C}$. To the jacket was added $\mathrm{Et}_{2} \mathrm{O}(2.0 \mathrm{~mL})$ at $-78{ }^{\circ} \mathrm{C}$ and the apparatus was warmed to $-20{ }^{\circ} \mathrm{C}$. The apparatus was kept at $-20{ }^{\circ} \mathrm{C}$ till single crystals suitable for X-ray analysis were obtained (from $12 \mathrm{~h}$ to $48 \mathrm{~h}$ ). 


\section{Computational Results}

Calculated Transition State Structures for the Addition of a Trichlorosilyl Enolate to Propiophenone.

Calculations carried out at the PM3 level of theory using the PC version of the GAMESS US (QC) Package. All energies are in Hartrees, the vibrational modes of the transition states are also given.

equatorial-equatorial isomer vi-Re

COORDINATES OF SYMMETRY UNIQUE ATOMS (ANGS)

ATOM CHARGE X $\quad$ Y $\quad$ Z

C $\quad \begin{array}{lllll}6.0 & 3.6736751761 & -0.1665324472 & -0.4381274465\end{array}$

C $\quad \begin{array}{llllll}6.0 & 4.4431757752 & -0.7403512367 & -1.4688399261\end{array}$

C $\quad 6.0 \quad 3.9813260866-1.8410505735$-2.1641364776

C $\quad \begin{array}{lllll}6.0 & 2.7825942047 & -2.4682906903 & -1.7848544066\end{array}$

$\begin{array}{lllll}\text { C } & 6.0 & 2.0700908243 & -1.9514082812 & -0.6970255478\end{array}$

C $\quad 6.0 \quad 0.9175969128-2.6722030214 \quad-0.1050264752$

C $\quad \begin{array}{lllll}6.0 & 1.1286996720 & -3.9298764936 & 0.4820901694\end{array}$

C $\quad \begin{array}{lllll}6.0 & 0.0219255736 & -4.6622779944 & 0.9273259989\end{array}$

C $\quad 6.0-1.2479647554-4.1268224853 \quad 0.8202352794$

C $\quad 6.0-1.4440604200 \quad-2.8206238899 \quad 0.3378166353$

C $\quad \begin{array}{lllll}6.0 & 4.1767481687 & 1.0942487469 & 0.2764304807\end{array}$

C $\quad \begin{array}{lllll}6.0 & 5.1960676631 & 0.7584542460 & 1.4163821457\end{array}$

C $\quad \begin{array}{lllll}6.0 & 4.5553888626 & -0.1954927234 & 2.4243268813\end{array}$

C $\quad \begin{array}{lllll}6.0 & 6.4836140246 & 0.1332202355 & 0.8870219099\end{array}$

C $\quad \begin{array}{lllll}6.0 & 5.5389024040 & 2.0622193659 & 2.1427675709\end{array}$

C $\quad \begin{array}{llllll}6.0 & 4.0331121460 & 2.9422659229 & -1.2253071075\end{array}$

C $\quad \begin{array}{lllll}6.0 & 2.3192759288 & -3.6353991674 & -2.5752404011\end{array}$

$\begin{array}{lllll}\text { C } & 6.0 & 2.4871103778 & -4.5031157741 & 0.6593886701\end{array}$ 

C $\quad 6.0-2.8603311255-2.2341695427 \quad 0.3730936456$
C $\quad \begin{array}{lllll}6.0 & -3.7043097847 & -2.6065145595 & -0.8916995486\end{array}$
C $\quad 6.0 \quad-3.0396974782 \quad-2.0522711919-2.1507490089$
C $\quad 6.0-3.8829325781 \quad-4.1144887719-1.0451880660$
C $\quad \begin{array}{lllll}6.0 & -5.0838502647 & -1.9566725125 & -0.7523264315\end{array}$
C $\quad \begin{array}{lllll} & 6.0 & -3.7892958306 & -1.8604342663 & 2.5538332032\end{array}$
CL $\quad \begin{array}{lllll} & 17.0 & -0.8351334021 & -0.0234385257 & 2.1544354287\end{array}$
$\begin{array}{lllll}\mathrm{N} & 7.0 & 2.4643756804 & -0.7369505875 & -0.1289856514\end{array}$
N $\quad \begin{array}{lllll}7.0 & -0.3545330462 & -2.0945919484 & -0.0956326203\end{array}$
$\begin{array}{llllll}\text { O } & 8.0 & 1.6135325468 & -0.1680098841 & 0.7753199886\end{array}$
$\begin{array}{lllll}\mathrm{O} & 8.0 & -0.5034183149 & -0.8423938891 & -0.5855344174\end{array}$
$\begin{array}{llllll}\mathrm{O} & 8.0 & 4.8334201824 & 1.9199112026 & -0.6803113745\end{array}$
$\begin{array}{lllll}\text { O } & 8.0 & -3.5103604147 & -2.7779485196 & 1.5252304020\end{array}$
SI $\quad \begin{array}{lllll}14.0 & 0.1380038104 & 0.6203662054 & 0.1867434572\end{array}$
$\begin{array}{lllll}\text { C } & 6.0 & -2.0862862912 & 2.1448599105 & -0.6178583033\end{array}$
$\begin{array}{llllll}\text { C } & 6.0 & -3.4935996747 & 2.1542912517 & -0.1477539752\end{array}$
C $\quad 6.0-1.5938163660 \quad 3.1327035316-1.6608526626$
$\begin{array}{lllll}\text { C } & 6.0 & -3.9130456871 & 1.1875546031 & 0.7750379619\end{array}$
C $\quad \begin{array}{lllll}6.0 & -4.4204016186 & 3.1038728667 & -0.5947249276\end{array}$
$\begin{array}{lllll}\text { C } & 6.0 & -5.2270372192 & 1.1530808641 & 1.2210068612\end{array}$
$\begin{array}{lllll}\text { C } & 6.0 & -5.7292867591 & 3.0800101963 & -0.1297183105\end{array}$
$\begin{array}{lllll}\text { C } & 6.0 & -6.1388073436 & 2.1044893797 & 0.7761087320\end{array}$
$\begin{array}{lllll}\text { O } & 8.0 & -1.4319188712 & 1.0545197407 & -0.4339004364\end{array}$
$\begin{array}{lllll}\text { C } & 6.0 & -0.0192128017 & 3.1947903124 & 0.9090313862\end{array}$
$\begin{array}{llllll}\text { O } & 8.0 & 0.7275824487 & 2.1189115473 & 0.8182887928\end{array}$
$\begin{array}{lllll}\text { O } & 8.0 & 0.8101313079 & 4.1769391621 & 0.4669069558\end{array}$
$\begin{array}{lllll}\mathrm{C} & 6.0 & 0.2371105254 & 5.4516674858 & 0.2560570104\end{array}$
$\begin{array}{lllll}\text { C } & 6.0 & -1.3480415057 & 3.2583810158 & 1.2502773790\end{array}$
$\begin{array}{lllll}\text { C } & 6.0 & -2.3366167040 & 2.9979004367 & -2.9732606212\end{array}$
CL $\quad \begin{array}{llllll} & 17.0 & 1.1001726165 & 1.0681218959 & -1.8747287301\end{array}$
$\begin{array}{lllll}\mathrm{H} & 1.0 & 5.4050027658 & -0.2731513365 & -1.7337554766\end{array}$ 


\begin{tabular}{|c|c|c|c|c|}
\hline $\mathrm{H}$ & 1.0 & 4.5511223739 & -2.2373122199 & -3.0155421640 \\
\hline $\mathrm{H}$ & 1.0 & 0.1645957685 & -5.6596126095 & 1.3658331949 \\
\hline $\mathrm{H}$ & 1.0 & -2.1222760737 & -4.7149749253 & 1.1423383235 \\
\hline $\mathrm{H}$ & 1.0 & 3.3108008547 & 1.6416586923 & 0.7369552908 \\
\hline $\mathrm{H}$ & 1.0 & 5.2169061076 & -0.3508640837 & 3.2879418054 \\
\hline $\mathrm{H}$ & 1.0 & 4.3627146863 & -1.1851418216 & 1.9879361109 \\
\hline $\mathrm{H}$ & 1.0 & 3.6032752401 & 0.1962750291 & 2.8077636243 \\
\hline $\mathrm{H}$ & 1.0 & 7.1958348669 & -0.0420379205 & 1.7051451956 \\
\hline $\mathrm{H}$ & 1.0 & 6.9823392185 & 0.7802273282 & 0.1529637520 \\
\hline $\mathrm{H}$ & 1.0 & 6.2966190409 & -0.8364441472 & 0.4052223466 \\
\hline $\mathrm{I}$ & 1.0 & 6.0225975907 & 2.7850119986 & 1.4724265650 \\
\hline $\mathrm{H}$ & 1.0 & 6.2293335566 & 1.8768024621 & 2.9769048022 \\
\hline $\mathrm{H}$ & 1.0 & 4.6414193182 & 2.5400728531 & 2.5583128695 \\
\hline $\mathrm{H}$ & 1.0 & 3.7626814761 & 3.6856522419 & -0.4661568218 \\
\hline $\mathrm{H}$ & 1.0 & 3.1244209181 & 2.5521131851 & -1.7052714274 \\
\hline $\mathrm{H}$ & 1.0 & 4.6985436568 & 3.3892333862 & -1.9682475086 \\
\hline $\mathrm{H}$ & 1.0 & 2.0839800639 & -3.3179213681 & -3.6020313068 \\
\hline $\mathrm{H}$ & 1.0 & 1.4180034062 & -4.1065838052 & -2.1607860348 \\
\hline $\mathrm{H}$ & 1.0 & 3.1011765852 & -4.4062857873 & -2.6398449536 \\
\hline $\mathrm{H}$ & 1.0 & 3.2129623275 & -3.7386078005 & 0.9700672338 \\
\hline 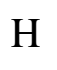 & 1.0 & 2.8501288211 & -4.9458105997 & -0.2810638686 \\
\hline $\mathrm{H}$ & 1.0 & 2.4972333925 & -5.2951892073 & 1.4215002485 \\
\hline $\mathrm{H}$ & 1.0 & -2.7949960253 & -1.1086764846 & 0.4498420370 \\
\hline $\mathbf{L}$ & 1.0 & -2.8539310385 & -0.9720826487 & -2.0717844218 \\
\hline 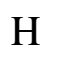 & 1.0 & -3.6764887261 & -2.2126186002 & -3.0319396398 \\
\hline $\mathrm{H}$ & 1.0 & -2.0800111495 & -2.5437833101 & -2.3570918873 \\
\hline $\mathrm{H}$ & 1.0 & -4.5151702283 & -4.3451778336 & -1.9134905793 \\
\hline $\mathrm{H}$ & 1.0 & -4.3614194503 & -4.5620709366 & -0.1635064578 \\
\hline $\mathrm{H}$ & 1.0 & -2.9209330437 & -4.6232867998 & -1.196397634 \\
\hline $\mathrm{H}$ & 1.0 & -5.6804629343 & -2.0989006382 & -1.6633667930 \\
\hline & 1. & -5.0039886265 & -0.8737492329 & -0.5709979334 \\
\hline
\end{tabular}




$\begin{array}{lllll}\mathrm{H} & 1.0 & -5.6537221879 & -2.3886521438 & 0.0816701713 \\ \mathrm{H} & 1.0 & -4.5824740136 & -1.1504202832 & 2.2585298916 \\ \mathrm{H} & 1.0 & -2.8983837558 & -1.3065304137 & 2.8834363833 \\ \mathrm{H} & 1.0 & -4.1539847186 & -2.5100332969 & 3.3536474910 \\ \mathrm{H} & 1.0 & -1.6731000971 & 4.1720072028 & -1.2615427433 \\ \mathrm{H} & 1.0 & -0.5051139423 & 2.9712010252 & -1.8484800056 \\ \mathrm{H} & 1.0 & -3.1885764651 & 0.4427965345 & 1.1622806304 \\ \mathrm{H} & 1.0 & -4.1193232624 & 3.8560703575 & -1.3405592544 \\ \mathrm{H} & 1.0 & -5.5194230919 & 0.3549438303 & 1.9232228459 \\ \mathrm{H} & 1.0 & -6.4445649562 & 3.8315141041 & -0.4830942492 \\ \mathrm{H} & 1.0 & -7.1739948066 & 2.0864425300 & 1.1350527754 \\ \mathrm{H} & 1.0 & -0.5924572260 & 5.3883168896 & -0.4681052707 \\ \mathrm{H} & 1.0 & 1.0686324192 & 6.0257891599 & -0.1591404109 \\ \mathrm{H} & 1.0 & -0.1141610237 & 5.9047324930 & 1.1894118529 \\ \mathrm{H} & 1.0 & -1.8777055294 & 4.2055756094 & 1.3154875844 \\ \mathrm{H} & 1.0 & -1.7905434242 & 2.4616888847 & 1.8558535740 \\ \mathrm{H} & 1.0 & -1.8648407030 & 3.6180858425 & -3.7462499581 \\ \mathrm{H} & 1.0 & -3.3846425811 & 3.3265356096 & -2.8800688391 \\ \mathrm{H} & 1.0 & -2.3384315627 & 1.9627261319 & -3.3381525904\end{array}$

--- OPTIMIZED RHF MO-S --- GENERATED AT 14:16:05 LT 7-SEP-2004 $\mathrm{E}=-298.9173559756, \mathrm{E}(\mathrm{NUC})=3622.4495439031$ MODE 1 FREQUENCY $=-356.99789\left(\mathrm{CM}^{* *}-1\right)$ 
equatorial-equatorial isomer vi-Si

\section{COORDINATES OF SYMMETRY UNIQUE ATOMS (ANGS)}




\begin{tabular}{|c|c|c|c|}
\hline $\mathrm{N}$ & $7.0 \quad 0.2078720414$ & -2.2763950688 & 0.1071844307 \\
\hline $\mathrm{O}$ & $8.0-1.5619738856$ & -0.1360169146 & -0.7539036067 \\
\hline $\mathrm{O}$ & $8.0 \quad 0.5533878146$ & -1.0311327182 & 0.5055788397 \\
\hline $\mathrm{O}$ & $8.0-4.3149013292$ & 2.4425502944 & 0.8495968476 \\
\hline $\mathrm{O}$ & $8.0 \quad 3.2202475688$ & -3.4849431725 & -1.5042923314 \\
\hline SI & $\begin{array}{ll}14.0 & 0.0596337071\end{array}$ & 0.4496721386 & -0.3368957710 \\
\hline $\mathrm{CL}$ & $17.0-0.6260397273$ & 31.1581714740 & $0 \quad 1.7506629569$ \\
\hline $\mathrm{C}$ & $6.0 \quad 2.6852991643$ & 1.4947821756 & -0.2534111058 \\
\hline $\mathrm{C}$ & $6.0 \quad 3.0886278351$ & 2.6948040147 & 0.5118109046 \\
\hline $\mathrm{C}$ & $6.0 \quad 3.6487048610$ & 0.7549930143 & -1.1720527644 \\
\hline $\mathrm{C}$ & $6.0 \quad 2.3485946776$ & 3.1055752131 & 1.6300036248 \\
\hline $\mathrm{C}$ & $6.0 \quad 4.2124220682$ & 3.4414978782 & 0.1293034428 \\
\hline $\mathrm{C}$ & $6.0 \quad 2.7322156757$ & 4.2352712530 & 2.3440490056 \\
\hline $\mathrm{C}$ & $6.0 \quad 4.5832944081$ & 4.5724400463 & 0.8411631170 \\
\hline $\mathrm{C}$ & $6.0 \quad 3.8439840308$ & 4.9722639949 & 1.9519854395 \\
\hline $\mathrm{O}$ & $8.0 \quad 1.6804644762$ & 0.8145611625 & 0.1651211917 \\
\hline $\mathrm{C}$ & $6.0 \quad 0.4610426799$ & 2.8693467701 & -1.4351599949 \\
\hline $\mathrm{O}$ & $8.0-0.3924410248$ & 1.9532543033 & -1.0491138864 \\
\hline $\mathrm{O}$ & $8.0-0.1389367108$ & 4.0226403778 & -1.0446443656 \\
\hline $\mathrm{C}$ & $\begin{array}{ll}6.0 & 0.4317406988\end{array}$ & 5.2343602076 & -1.5021724815 \\
\hline $\mathrm{C}$ & $6.0 \quad 1.7082987849$ & 2.6864753074 & -1.9819375250 \\
\hline $\mathrm{C}$ & $6.0 \quad 5.0179095484$ & 0.5207129595 & -0.5784454387 \\
\hline $\mathrm{H}$ & $1.0-5.1061212018$ & 0.3914896430 & 2.0430525170 \\
\hline $\mathrm{H}$ & $1.0-4.4386575873$ & -1.6338280698 & 3.3400413228 \\
\hline $\mathrm{H}$ & $1.0-0.8765416721$ & -5.7717287875 & -1.1967788820 \\
\hline $\mathrm{H}$ & $1.0 \quad 1.5305191020$ & -5.1635624248 & -1.0884176824 \\
\hline $\mathrm{H}$ & $1.0-2.9804525660$ & 1.8940041606 & -0.6753794038 \\
\hline $\mathrm{H}$ & $1.0-5.3669356625$ & 0.1027135632 & -2.9563742134 \\
\hline $\mathrm{H}$ & $1.0-4.5267474939$ & -0.7952379338 & -1.6899786584 \\
\hline $\mathrm{H}$ & $1.0-3.6559781849$ & 0.4283114966 & -2.6392327240 \\
\hline $\mathrm{H}$ & $1.0-7.1356894609$ & 0.7643152924 & -1.2371263118 \\
\hline
\end{tabular}




\begin{tabular}{|c|c|c|c|}
\hline & $1.0-6.6690362292$ & 1.5988577363 & 0.2 \\
\hline $\mathrm{H}$ & $1.0-6.2493668960$ & -0.1093405756 & 0.0198507273 \\
\hline $\mathrm{H}$ & $1.0-5.5528515482$ & 3.3918210826 & -1.2 \\
\hline & $.0-6.0206632441$ & 2.4673106577 & -2.67714921 \\
\hline & $1.0-4.3232928487$ & 2.9052571152 & -2.424964875 \\
\hline & $1.0-3.0656120268$ & 4.0449748595 & 0.425782994 \\
\hline & $1.0-2.4403401309$ & 2.8961379014 & 1.657776 \\
\hline & $1.0-3.8623531703$ & 3.9386304398 & 2.029972 \\
\hline & $1.0-2.1213953439$ & -3.0413698345 & 3.79 \\
\hline & $1.0-1.6709240706$ & -3.9542925454 & 2.341480803 \\
\hline & $1.0-3.3418844053$ & -4.0038592636 & 2.939150093 \\
\hline & $1.0-3.5992825028$ & -3.4451325381 & -0.73924410 \\
\hline & $1.0-3.3512896284$ & -4.6067148711 & 0.581346259 \\
\hline $\mathrm{H}$ & $1.0-3.1362408013$ & -5.1160349582 & -1.103906605 \\
\hline & $1.0 \quad 2.7728378360$ & -1.6921984510 & -0.497 \\
\hline & $\begin{array}{ll}1.0 & 2.8040482607\end{array}$ & -1.5135465226 & 2.0388741 \\
\hline & $1.0 \quad 3.4530587694$ & -2.8290643503 & 3.0310677697 \\
\hline & $1.0 \quad 1.8268072557$ & -2.9609926212 & 2.3599487659 \\
\hline & 1.03 .9604121429 & -5.0927139460 & 1.97 \\
\hline & $1.0 \quad 3.8149348138$ & -5.3185574820 & 0.2210276120 \\
\hline F & $1.0 \quad 2.3588824633$ & -5.1538106069 & 1.2207513163 \\
\hline I & $1.0 \quad 5.4696933636$ & -3.1357281065 & 1.6558187937 \\
\hline & $1.0 \quad 4.9753002577$ & -1.7397331045 & 0.6786229822 \\
\hline & $1.0 \quad 5.3763321106$ & -3.28 & -0.104 \\
\hline I & $1.0 \quad 4.3094135672$ & -1.99531 & -2.4400 \\
\hline F & $1.0 \quad 2.5865118059$ & -2.1043503252 & -2.948627873 \\
\hline & $1.0 \quad 3.7481626915$ & -3.4099000697 & -3.38902817 \\
\hline & $1.0 \quad 3.1966132884$ & -0.2338731918 & -1.450698415 \\
\hline & $1.0 \quad 3.7251852463$ & 1.3154794774 & -2.129736965 \\
\hline & $1.0 \quad 1.4551383548$ & 2.5391154372 & 1.9492092 \\
\hline & $1.0 \quad 4.8188862818$ & 3.1086081246 & -0.726977 \\
\hline
\end{tabular}




$\begin{array}{lllll}\mathrm{H} & 1.0 & 2.1505998927 & 4.5439695340 & 3.2202602324 \\ \mathrm{H} & 1.0 & 5.4627815441 & 5.1482090264 & 0.5317708446 \\ \mathrm{H} & 1.0 & 4.1410265942 & 5.8635846503 & 2.5161867924 \\ \mathrm{H} & 1.0 & 1.5174409078 & 5.1652519932 & -1.6692393053 \\ \mathrm{H} & 1.0 & 0.2086227008 & 5.9472610352 & -0.7049169376 \\ \mathrm{H} & 1.0 & -0.0655975498 & 5.5323386081 & -2.4304744724 \\ \mathrm{H} & 1.0 & 2.3226596036 & 3.5606590101 & -2.2054103223 \\ \mathrm{H} & 1.0 & 1.9391137951 & 1.7988868736 & -2.5786276111 \\ \mathrm{H} & 1.0 & 5.6338237665 & -0.0714344171 & -1.2697283089 \\ \mathrm{H} & 1.0 & 4.9659129523 & -0.0399124077 & 0.3697101342 \\ \mathrm{H} & 1.0 & 5.5485008797 & 1.4673331298 & -0.3898478292\end{array}$

--- OPTIMIZED RHF MO-S --- GENERATED AT 12:43:45 LT 7-SEP-2004

$\mathrm{E}=-298.9155322280, \mathrm{E}(\mathrm{NUC})=3606.2832155595$

MODE 1 FREQUENCY $=-376.97244\left(\mathrm{CM}^{* *}-1\right)$

equatorial-axial isomer vii-Re

COORDINATES OF SYMMETRY UNIQUE ATOMS (ANGS)

\begin{tabular}{|c|c|c|c|}
\hline T & CHA & $\mathbf{Y}$ & $\mathbf{Z}$ \\
\hline $\mathrm{C}$ & $6.0-3.5144370835$ & 0.3143227851 & 0.38018123 \\
\hline $\mathrm{C}$ & $6.0-4.3711870244$ & -0.2234067866 & 1.354260565 \\
\hline $\mathrm{C}$ & $6.0-4.1322108733$ & -1.4736135316 & 1.895 \\
\hline $\mathrm{C}$ & $6.0-3.0755535054$ & -2.2580262308 & 1.4145784731 \\
\hline $\mathrm{C}$ & $6.0-2.2759087695$ & -1.7517062254 & 0.3792169477 \\
\hline $\mathrm{C}$ & $6.0-1.2403744706$ & -2.5946343232 & -0 \\
\hline $\mathrm{C}$ & $6.0-1.5989232626$ & -3.7530762296 & -0.9678314513 \\
\hline$r$ & $6.0-0.5842599625$ & -4.5972070481 & -1.4409988231 \\
\hline & $6.0 \quad 0.7411120599$ & -4.2897681152 & -1.2002251 \\
\hline & $6.0 \quad 1.0965715772$ & -3.0872433990 & -0.562784525 \\
\hline
\end{tabular}




\begin{tabular}{|c|c|c|c|}
\hline $\mathrm{C}$ & $6.0-3.7450059882$ & 1.7375315750 & -0.1336852162 \\
\hline $\mathrm{C}$ & $6.0-5.0794157870$ & 1.8861729438 & -0.9396382692 \\
\hline$C$ & $6.0-5.2663811812$ & 0.6868136802 & -1.8670879351 \\
\hline$C$ & $6.0-6.3033936595$ & 2.0245114147 & -0.0373992216 \\
\hline$r$ & $6.0-4.9708849686$ & 3.1460036702 & -1.8050148423 \\
\hline $\mathrm{C}$ & $6.0-3.1546021512$ & 3.8106987225 & 0.8913207719 \\
\hline $\mathrm{C}$ & $\begin{array}{ll}6.0 & 0.4679340307\end{array}$ & 1.5581168430 & 1.9919978788 \\
\hline $\mathrm{C}$ & $\begin{array}{ll}6.0 & -2.8366985199\end{array}$ & -3.5917780824 & 2.0207637920 \\
\hline $\mathrm{C}$ & $\begin{array}{lll}6.0 & -3.0058925527\end{array}$ & -4.1228679877 & -1.2621550420 \\
\hline $\mathrm{C}$ & $6.0 \quad 2.5816207841$ & -2.7754742468 & -0.3395241164 \\
\hline $\mathrm{C}$ & $6.0 \quad 3.1354442070$ & -3.4589590812 & 0.9577336672 \\
\hline $\mathrm{C}$ & 6.02 .4857796418 & -2.8054400504 & 2.1789306382 \\
\hline $\mathrm{C}$ & $6.0 \quad 2.8769395279$ & -4.9621371536 & 0.9879301855 \\
\hline $\mathrm{C}$ & 6.04 .6466816394 & -3.2303838231 & 1.0447911571 \\
\hline $\mathrm{C}$ & 6.03 .5966374193 & -2.2871254067 & -2.4399361292 \\
\hline $\mathrm{CL}$ & $17.0-0.5241497753$ & 32.5968175136 & $6-1.1851088709$ \\
\hline $\mathrm{O}$ & $8.0-0.2104392687$ & 0.7988841615 & 1.1735999543 \\
\hline $\mathrm{N}$ & $7.0-2.4408599722$ & -0.4340976178 & -0.0490419244 \\
\hline $\mathrm{N}$ & $7.0 \quad 0.1032337306$ & -2.2155385070 & -0.1802436727 \\
\hline $\mathrm{O}$ & $8.0-1.5523737716$ & 0.0585340085 & -0.9330573509 \\
\hline $\mathrm{O}$ & $8.0 \quad 0.3909183869$ & -1.0008660593 & 0.3330805901 \\
\hline $\mathrm{O}$ & $8.0-3.7359157503$ & 2.5394576719 & 1.0522644538 \\
\hline $\mathrm{O}$ & $8.0 \quad 3.3148164489$ & -3.2597019266 & -1.4607804751 \\
\hline SI & $14.0 \quad 0.1201554754$ & 0.5483556038 & -0.5161554736 \\
\hline $\mathrm{C}$ & $6.0 \quad 2.6411787524$ & 1.6707087431 & 0.2606217840 \\
\hline $\mathrm{C}$ & $6.0 \quad 3.2357368609$ & 2.9466445653 & -0.2076573872 \\
\hline $\mathrm{C}$ & $6.0 \quad 3.3798998382$ & 0.8233857704 & 1.2897754864 \\
\hline $\mathrm{C}$ & $6.0 \quad 2.6346080392$ & 3.6757173096 & -1.2425211108 \\
\hline $\mathrm{C}$ & $6.0 \quad 4.4129123777$ & 3.4449757566 & 0.3708937259 \\
\hline $\mathrm{C}$ & $6.0 \quad 3.1954508161$ & 4.8707337233 & -1.6796473617 \\
\hline $\mathrm{C}$ & $6.0 \quad 4.9645133337$ & 4.6400980158 & -0.0654086986 \\
\hline
\end{tabular}


C $\quad \begin{array}{lllll}6.0 & 4.3563266652 & 5.3587890906 & -1.0919449166\end{array}$

$\begin{array}{llllll}\text { O } & 8.0 & 1.8003392611 & 1.0654654964 & -0.4946246254\end{array}$

$\begin{array}{lllll}\mathrm{O} & 8.0 & 0.4027432842 & 0.8760888285 & 3.1681254614\end{array}$

C $\quad \begin{array}{lllll}6.0 & 1.1991464438 & 2.6840462414 & 1.6926694427\end{array}$

C $\quad \begin{array}{lllll}6.0 & 0.8940512092 & 1.5380022396 & 4.3182086557\end{array}$

$\begin{array}{lllll}\text { CL } & 17.0 & 0.6346181680 & -0.3366168960 & -2.5168228844\end{array}$

C $\quad \begin{array}{lllll}6.0 & 4.7173677707 & 0.3558246666 & 0.7525009813\end{array}$

$\mathrm{H} \quad \begin{array}{lllll}1.0 & -5.2345192707 & 0.3841992561 & 1.6824095123\end{array}$

$\mathrm{H} \quad 1.0-4.7734932181 \quad-1.85629063602 .7014010333$

$\mathrm{H} \quad 1.0 \quad-0.8506934785 \quad-5.5060931356 \quad-1.9972905419$

H $\quad \begin{array}{llllll}1.0 & 1.5388954749 & -4.9751042461 & -1.5274635706\end{array}$

H $\quad \begin{array}{llll}1.0 & -2.9012081706 & 2.0466415623 & -0.8167766722\end{array}$

H $\quad \begin{array}{lllll}1.0 & -6.1418434276 & 0.8272067436 & -2.5161184636\end{array}$

H $\quad \begin{array}{lllll}1.0 & -5.4272523292 & -0.2422352320 & -1.3036009148\end{array}$

H $\quad \begin{array}{lllll}1.0 & -4.3960778086 & 0.5410634793 & -2.5208309184\end{array}$

H $\quad \begin{array}{lllll}1.0 & -7.2278059222 & 2.0487260372 & -0.6303379192\end{array}$

H $\quad$\begin{tabular}{lllll}
\hline & 1.0 & -6.2673796266 & 2.9500103731 & 0.5529662552
\end{tabular}

H $\quad \begin{array}{llll}1.0 & -6.3895849108 & 1.1858434254 & 0.6715326809\end{array}$

H $\quad \begin{array}{lllll}1.0 & -4.7454059898 & 4.0349169444 & -1.1923084449\end{array}$

H $\quad \begin{array}{llll}1.0 & -5.9109820891 & 3.3406400858 & -2.3378587109\end{array}$

H $\quad \begin{array}{lllll}1.0 & -4.1773559274 & 3.0541306153 & -2.5592855582\end{array}$

$\mathrm{H} \quad \begin{array}{lllll}1.0 & -3.7333959296 & 4.4251037431 & 0.1848123178\end{array}$

H $\quad \begin{array}{llll}1.0 & -2.1084884778 & 3.7499072013 & 0.5581292273\end{array}$

H $\quad \begin{array}{lllll}1.0 & -3.2158661470 & 4.2293495211 & 1.8984430697\end{array}$

H $\quad \begin{array}{lllll}1.0 & -3.1777459098 & -3.6255504985 & 3.0650606407\end{array}$

H $\quad 1.0-1.7727889416 \quad-3.8645515787 \quad 2.0112511832$

H $\quad \begin{array}{lllll}1.0 & -3.3849794401 & -4.3704336469 & 1.4681610177\end{array}$

H $\quad 1.0 \quad-3.7349494374 \quad-3.4003575576 \quad-0.8716056805$

$\mathrm{H} \quad 1.0 \quad-3.2497487036-5.1069186586-0.8349525436$

H $\quad 1.0 \quad-3.1568814534-4.1897073104-2.3497565607$

$\mathrm{H} \quad 1.0 \quad 2.7291573046 \quad-1.6666116582 \quad-0.2346167041$ 


\begin{tabular}{|c|c|c|c|}
\hline & .0 & $2.5977044339-1.7060355473$ & 2.15960 \\
\hline & .0 & $.9457874395-3.1661363220$ & 3.1089626669 \\
\hline & & $4132383084-3.0296358029$ & 2.2436394661 \\
\hline & 0 & $3.3058753862-5.4142342478$ & 1.8928069143 \\
\hline & .0 & $3.3284805673-5.4667804466$ & 0.1228968954 \\
\hline & 0 & $1.8027979159-5.1931517332$ & 0.9873 \\
\hline & 1.0 & $5.0553371654-3.6662232058$ & 1.967 \\
\hline & 0 & $4.8952111638-2.1558446426$ & 1.05 \\
\hline & 1.0 & $5.1812479872-3.6911204583$ & 0.20 \\
\hline & 1.0 & $4.3091893902-1.5421494057$ & -2.0635256830 \\
\hline & 1.0 & $2.6925102787-1.7807609392$ & -2.8078465963 \\
\hline & 1.0 & $4.0575685453-2.8782395106$ & -3.23 \\
\hline & 1.0 & $2.7694131696-0.0655681812$ & 1.58 \\
\hline & 1.0 & $3.5170225857 \quad 1.4078671817$ & 06462 \\
\hline & 1.0 & $1.7036040882 \quad 3.3130325022$ & -1.7104220212 \\
\hline & 1.0 & $4.9294313251 \quad 2.8716348757$ & 1.1557932664 \\
\hline & 1.0 & $2.7141450714 \quad 5.4283446752$ & -2.49 \\
\hline & 1.0 & $5.8838032337 \quad 5.017146$ & 0.396 \\
\hline & 1.0 & $4.7941854598 \quad 6.3028221222$ & -1.4352557691 \\
\hline & 1.0 & $1.7557872961 \quad 3.1919713620$ & 2.4813703260 \\
\hline & 1.0 & $0.9187273390 \quad 3.3214871616$ & 0.8488279785 \\
\hline & 1.0 & $1.1657121636 \quad 0.7209741023$ & 4.9904909379 \\
\hline & 1.0 & $0.0983500790 \quad 2.1464391041$ & 4.7596393564 \\
\hline & 1.0 & $1.7671105493 \quad 2.1726603417$ & 4.102980449 \\
\hline & 1.0 & $5.1207564257-0.4680315825$ & 1.3638510604 \\
\hline & 1.0 & $5.4550392642 \quad 1.1745742439$ & 0.7586557886 \\
\hline & 1.0 & $4.6392121794-0.0177628766$ & $-0.27918910 \varsigma$ \\
\hline
\end{tabular}

--- OPTIMIZED RHF MO-S --- GENERATED AT 17:45:58 LT 7-SEP-2004

$\mathrm{E}=-298.9165585306, \mathrm{E}(\mathrm{NUC})=3610.1201541711$

MODE 1 FREQUENCY $=-382.24778\left(\mathrm{CM}^{* *}-1\right)$ 
equatorial-axial isomer vii-Si

\section{COORDINATES OF SYMMETRY UNIQUE ATOMS (ANGS)}

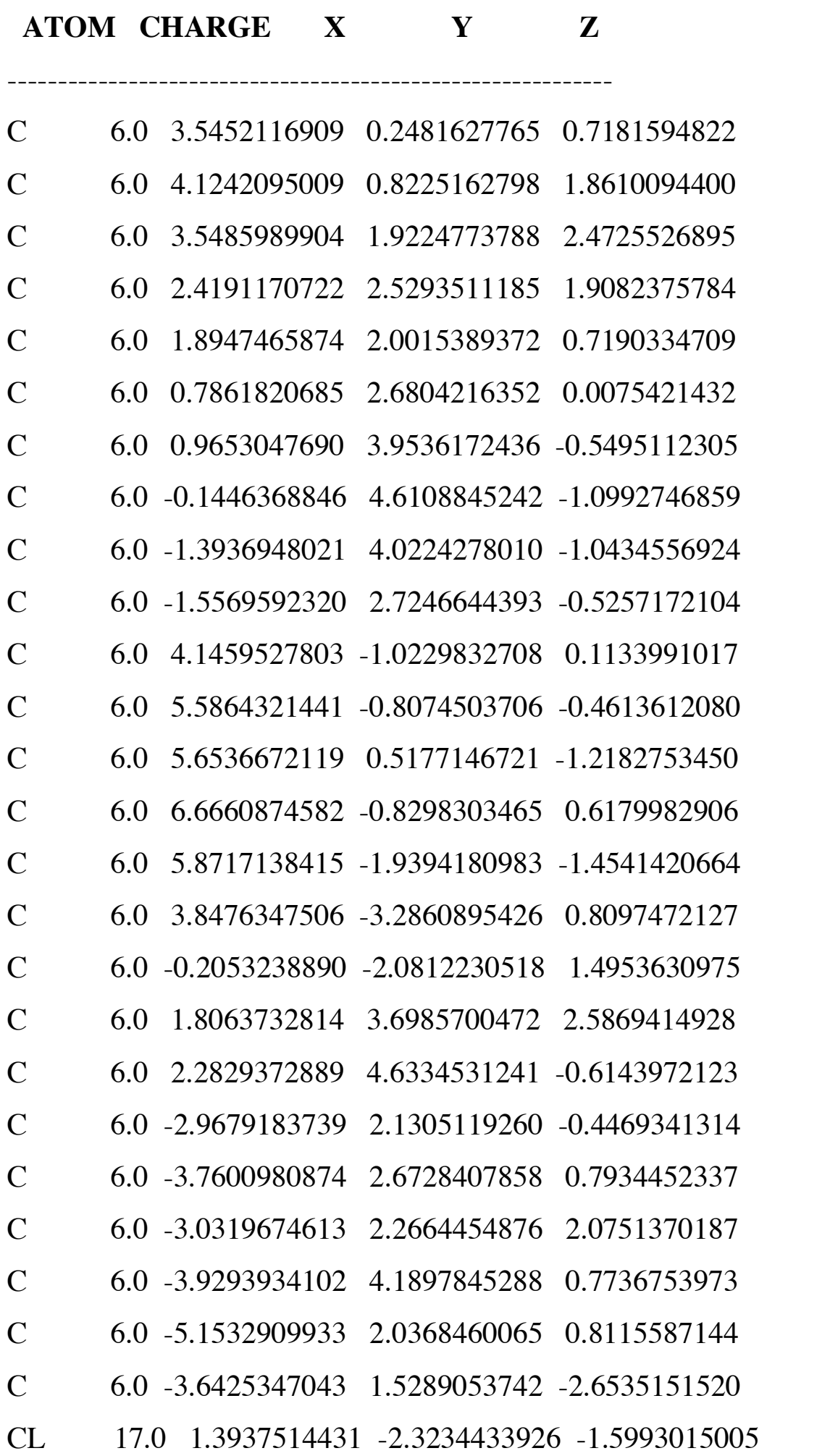




\begin{tabular}{|c|c|c|c|}
\hline $\mathrm{O}$ & $8.0 \quad 0.3532713585$ & -1.0459542875 & 0.9223157789 \\
\hline $\mathrm{N}$ & $7.0 \quad 2.4004740581$ & 0.8114230475 & 0.1985679118 \\
\hline $\mathrm{N}$ & $7.0-0.4436968718$ & 2.0261436550 & -0.1168745307 \\
\hline $\mathrm{O}$ & $8.0 \quad 1.7647670707$ & 0.2670030866 & -0.8561532729 \\
\hline $\mathrm{O}$ & $8.0-0.5055810316$ & 0.7188111713 & 0.2227369227 \\
\hline $\mathrm{O}$ & $8.0 \quad 4.1285860937$ & -1.9620309078 & 1.1940425249 \\
\hline $\mathrm{O}$ & $8.0-3.6807351394$ & 2.4904182049 & -1.6257644142 \\
\hline SI & $14.0 \quad 0.2032624111$ & -0.6037618788 & -0.7519508591 \\
\hline$C$ & $6.0-1.8493402812$ & -2.5731446121 & -0.7334478812 \\
\hline $\mathrm{C}$ & $6.0-3.1212160276$ & -2.6664186613 & 0.0314407733 \\
\hline $\mathrm{C}$ & $6.0-1.5027242939$ & -3.7079718707 & -1.6788084338 \\
\hline $\mathrm{C}$ & $6.0-3.7895702273$ & -1.5143225930 & 0.4549679452 \\
\hline $\mathrm{C}$ & $6.0-3.6825045876$ & -3.9177709556 & 0.3185292037 \\
\hline $\mathrm{C}$ & $6.0-4.9964181673$ & -1.6094951589 & 1.1367160506 \\
\hline $\mathrm{C}$ & $6.0-4.8829848357$ & -4.0099763911 & 1.0093885461 \\
\hline $\mathrm{C}$ & $6.0-5.5460045680$ & -2.8551551290 & 1.4178672130 \\
\hline $\mathrm{O}$ & $8.0-1.3525502851$ & -1.4074444626 & -0.9227714443 \\
\hline $\mathrm{O}$ & $8.0-0.5714309105$ & -1.5990939025 & 2.7115233413 \\
\hline $\mathrm{C}$ & $6.0-0.4733937719$ & -3.3102631173 & 0.9378661148 \\
\hline $\mathrm{C}$ & $6.0-1.0452713096$ & -2.5355588648 & 3.6610380044 \\
\hline $\mathrm{C}$ & $6.0-1.9100896710$ & -3.3768022905 & -3.0989188697 \\
\hline $\mathrm{CL}$ & $17.0-0.280433453$ & $4 \quad 0.416082057$ & $7-2.6919577588$ \\
\hline $\mathrm{H}$ & $1.0 \quad 5.0460010466$ & 0.3648988943 & 2.2647300592 \\
\hline $\mathrm{H}$ & $1.0 \quad 3.9790590843$ & 2.3235896851 & 3.4001829363 \\
\hline $\mathrm{H}$ & $1.0-0.0161264136$ & 5.5985961030 & -1.5622170972 \\
\hline $\mathrm{H}$ & $1.0-2.2776837181$ & 4.5557676630 & -1.4279427520 \\
\hline $\mathrm{H}$ & $1.0 \quad 3.4986318509$ & -1.3939266443 & -0.7340468576 \\
\hline $\mathrm{H}$ & $1.0 \quad 6.6256158874$ & 0.6339503426 & -1.7174232925 \\
\hline $\mathrm{H}$ & $1.0 \quad 5.5304914770$ & 1.3772427377 & -0.5455104300 \\
\hline $\mathrm{H}$ & $1.0 \quad 4.8791138995$ & 0.5803814250 & -1.9942835495 \\
\hline $\mathrm{H}$ & $1.0 \quad 7.6534244181$ & -0.6085595351 & 0.1901778636 \\
\hline
\end{tabular}




\begin{tabular}{|c|c|c|c|c|}
\hline & 1.0 & 6.7314140146 & -1.8126693487 & 1.103 \\
\hline & 1.0 & 6.4762593595 & -0.0850274737 & 1.4069498636 \\
\hline & 1.0 & 5.7454525124 & -2.9279873493 & -0.981664062 \\
\hline & 1.0 & 6.9006973417 & -1.8820539105 & -1.8 \\
\hline & 1.0 & 5.1985064928 & -1.8990435374 & -2.321496793 \\
\hline & 1.0 & 4.6286380498 & -3.6805545737 & 0.141797033 \\
\hline & 1.0 & 2.8647079432 & -3.3776241921 & 0.324 \\
\hline & 1.0 & 3.8582027568 & -3.8181000529 & 1.7637880707 \\
\hline & 1.0 & 2.0991040315 & 3.7541608494 & 77706 \\
\hline & 1.0 & 0.7086749737 & 3.6598817206 & 2.5492030999 \\
\hline & 1.0 & 2.1237486712 & 4.6368400154 & 2.1061932159 \\
\hline & 1.0 & 3.0941298073 & 4.0512999578 & -0.1570412845 \\
\hline & 1.0 & 2.2457887985 & 5.6094239076 & -0.1081222570 \\
\hline & 1.0 & 2.5588643038 & 4.8173416426 & -1.6633445958 \\
\hline & 1.0 & -2.910 & 1.0091252990 & -0.3 \\
\hline & 1.0 & -2.8653640744 & 1.1808088699 & 2.1207844726 \\
\hline & 1.0 & -3.6172042421 & 2.5464524873 & 2.9618907641 \\
\hline & 1.0 & -2.0551597586 & 2.7603648933 & 2.1672079290 \\
\hline & 1.0 & -4.5496730846 & 4.5241449690 & 1.6170783457 \\
\hline & 1.0 & -4.4185531283 & 4.5331112535 & -0.1479 \\
\hline & 1.0 & -2.9654886712 & 4.7104182296 & 0.8579321193 \\
\hline & 1.0 & -5.7402330592 & 2.4083657371 & 1.6632330187 \\
\hline & 1.0 & -5.1081829844 & 0.9382462000 & 0.9074914397 \\
\hline & 1.0 & -5.7199075104 & 2.2690251859 & -0.0997 \\
\hline & 1.0 & -4.1788064812 & 0.6169886714 & -2.364937535 \\
\hline & 1.0 & -2.6167031069 & 1.2754915280 & -2.9575928679 \\
\hline & 1.0 & -4.1705592532 & 2.0327926392 & -3.4670677108 \\
\hline & 1.0 & -2.0021329918 & -4.6423215751 & -1.3376893718 \\
\hline & 1.0 & -0.4074275987 & -3.9160830906 & -1.6228191089 \\
\hline & 1.0 & -3.3665247151 & -0.5178657633 & 0.243243390 \\
\hline & 1.0 & -3.1617932952 & -4.8289707468 & -0.016819 \\
\hline
\end{tabular}




$\begin{array}{lllll}\mathrm{H} & 1.0 & -5.5081123606 & -0.6818287075 & 1.4388507346 \\ \mathrm{H} & 1.0 & -5.3123857138 & -4.9944734653 & 1.2273180352 \\ \mathrm{H} & 1.0 & -6.4982472913 & -2.9285990566 & 1.9551283500 \\ \mathrm{H} & 1.0 & -0.9899765356 & -4.0660808016 & 1.5315826947 \\ \mathrm{H} & 1.0 & 0.1468935023 & -3.7051756751 & 0.1244646568 \\ \mathrm{H} & 1.0 & -1.7740613272 & -1.9725656362 & 4.2488564949 \\ \mathrm{H} & 1.0 & -0.2092569273 & -2.8510206770 & 4.2930501322 \\ \mathrm{H} & 1.0 & -1.5165093116 & -3.4186424152 & 3.2028906494 \\ \mathrm{H} & 1.0 & -1.6620743267 & -4.2085008377 & -3.7707334460 \\ \mathrm{H} & 1.0 & -1.3877979356 & -2.4840307915 & -3.4725072575 \\ \mathrm{H} & 1.0 & -2.9892914140 & -3.1949035774 & -3.1831402107\end{array}$

--- OPTIMIZED RHF MO-S --- GENERATED AT 15:34:44 LT 7-SEP-2004

$\mathrm{E}=-298.9181378177, \mathrm{E}(\mathrm{NUC})=3624.6715511984$

MODE 1 FREQUENCY $=-375.28748\left(\mathrm{CM}^{* *}-1\right)$

\section{Equatorial-Axial Isomer, Position 2a}

COORDINATES OF SYMMETRY UNIQUE ATOMS (ANGS)

\begin{tabular}{|c|c|c|c|}
\hline AT & CHARGE & $\mathbf{Y}$ & $\mathbf{Z}$ \\
\hline $\mathrm{C}$ & $6.0 \quad 1.9655831137$ & -2.5535 & -0.1822045977 \\
\hline $\mathrm{C}$ & $6.0 \quad 1.9737507198$ & -3.8484393873 & -0.7174315866 \\
\hline $\mathrm{C}$ & $6.0 \quad 0.7992716305$ & -4.5372919103 & -0.9609632489 \\
\hline $\mathrm{C}$ & $6.0-0.4214295391$ & -3.9786744768 & -0.5727416558 \\
\hline $\mathrm{C}$ & $6.0-0.4202125612$ & -2.7282233185 & 0.0707626910 \\
\hline $\mathrm{C}$ & $6.0-1.6911655065$ & -2.2200583394 & 0.6406482998 \\
\hline $\mathrm{C}$ & $6.0-2.3459133736$ & -2.8867563676 & 1.6827593231 \\
\hline $\mathrm{C}$ & $6.0-3.6306813357$ & -2.4578075998 & 2.0547669290 \\
\hline & $6.0-4.2309465965$ & -1.4005285125 & 1.398551823 \\
\hline
\end{tabular}



C $\quad 6.0-3.5330313692 \quad-0.6775818111 \quad 0.4121879583$
C $\quad \begin{array}{lllll}6.0 & 3.2895267870 & -1.7962119953 & -0.0132045614\end{array}$
C $\quad \begin{array}{lllll}6.0 & 4.1643209928 & -2.3613841172 & 1.1541110434\end{array}$
C $\quad \begin{array}{lllll}6.0 & 3.3324382290 & -2.3333522107 & 2.4383165963\end{array}$
C $\quad \begin{array}{lllll}6.0 & 4.6834418125 & -3.7804360675 & 0.9341024790\end{array}$
C $\quad \begin{array}{lllll}6.0 & 5.3697549633 & -1.4365398371 & 1.3475175221\end{array}$
C $\quad \begin{array}{llllll}6.0 & 4.6399379759 & -2.6060696782 & -1.8979972523\end{array}$
$\begin{array}{lllll}\text { C } & 6.0 & 1.2732964603 & 1.1019177593 & -2.2827877517\end{array}$
C $\quad 6.0-1.6774737988$-4.7172741319 -0.8578959341
C $\quad 6.0-1.7364763063 \quad-4.0105486824 \quad 2.4363017067$
$\begin{array}{lllll}\text { C } & 6.0 & -4.1899392957 & 0.5414839699 & -0.2490922478\end{array}$

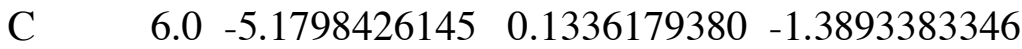
C $\quad 6.0-4.4449671043-0.7030989475-2.4365590182$
C $\quad 6.0-6.3833913429-0.6526542545 \quad-0.8776862722$
$\begin{array}{lllll}\text { C } & 6.0 & -5.6760055471 & 1.4166930010 & -2.0622147008\end{array}$
C $\quad \begin{array}{lllll}6.0 & -4.2343338179 & 2.3627548572 & 1.2849589421\end{array}$
CL $\quad \begin{array}{lllll}17.0 & -1.3177254063 & 2.5352255143 & -0.5517183452\end{array}$
$\begin{array}{llllll}\text { O } & 8.0 & 0.3415473460 & 0.3884057924 & -1.6980776229\end{array}$
N $\quad \begin{array}{lllll}7.0 & 0.7526248817 & -1.9750915540 & 0.1527801740\end{array}$
$\mathrm{N} \quad 7.0 \quad-2.2495060788 \quad-1.0562607167 \quad 0.1068297850$
$\begin{array}{lllll}\mathrm{O} & 8.0 & 0.7060933980 & -0.7256489280 & 0.6213004576\end{array}$
O $\quad 8.0-1.4710677208-0.3306480716-0.7413591895$
O $\quad 8.0 \quad 3.9209762731 \quad-1.5810549788 \quad-1.2633012678$
O $\quad \begin{array}{lllll}8.0 & -4.9133329562 & 1.2469318055 & 0.7548473503\end{array}$
SI $\quad \begin{array}{lllll}14.0 & -0.1789494268 & 0.6733870963 & -0.0674009675\end{array}$
$\begin{array}{llllll}\text { O } & 8.0 & 1.8824986214 & 0.1864788130 & -3.0790495019\end{array}$
$\begin{array}{lllll}\text { C } & 6.0 & 1.6082296236 & 2.4129854005 & -2.0266235887\end{array}$
C $\quad \begin{array}{lllll}6.0 & 2.9200613800 & 0.6405863558 & -3.9231601269\end{array}$
$\begin{array}{lllll}\text { CL } & 17.0 & -1.0640551991 & 0.8243204278 & 2.0312826339\end{array}$
C $\quad \begin{array}{lllll}6.0 & 2.0929627636 & 2.2887349896 & 0.2117210885\end{array}$
C $\quad \begin{array}{lllll}6.0 & 1.7420863688 & 3.6894999855 & 0.5223343557\end{array}$ 


\begin{tabular}{|c|c|c|c|c|}
\hline $\mathrm{C}$ & 6.0 & 3.5695291550 & 1.9557740679 & 0.07 \\
\hline $\mathrm{C}$ & 6.0 & 0.6431033186 & 4.0048705392 & 1.3285799998 \\
\hline $\mathrm{C}$ & 6.0 & 364700 & 471568 & 0.0337501239 \\
\hline $\mathrm{C}$ & 0 & 0.3815669335 & 5.3339996448 & 490 \\
\hline $\mathrm{C}$ & .0 & 2.2869271053 & 6.0405283072 & 344328 \\
\hline $\mathrm{C}$ & 5.0 & 1.1963458348 & 6.3492234376 & 1.1633245138 \\
\hline $\mathrm{O}$ & 8.0 & 1.3335582699 & 1.299 & 5821 \\
\hline $\mathrm{C}$ & 6.0 & 4.287 & 2.1 & 1.3 \\
\hline $\mathrm{H}$ & 0 & 2.954 & 7299 & 775 \\
\hline $\mathrm{H}$ & .0 & 0.8265253935 & -5.521182 & -1.448 \\
\hline $\mathrm{H}$ & 1.0 & -4.1549904989 & -2.9715792882 & 2.8718359838 \\
\hline $\mathrm{U}$ & 1.0 & -5.2520305598 & -1.0820461852 & 1.66 \\
\hline $\mathrm{H}$ & 1.0 & 3.0967659825 & -0.7040691630 & 0.2 \\
\hline $\mathbf{H}$ & 1.0 & 3.9443762894 & -2.6055 & 3.30 \\
\hline $\mathrm{H}$ & 1.0 & 2.4976104311 & -3.0450355656 & 2.3 \\
\hline $\mathrm{H}$ & 1.0 & 2.9171232620 & -1.3343281050 & 7171 \\
\hline U & 1.0 & 5.1005574417 & -4.1939690302 & 1.8622458656 \\
\hline U & 1.0 & 5.4852283871 & -3.8048823733 & 0.1771369427 \\
\hline II & 1.0 & 3.8937402668 & 916661 & 0.5 \\
\hline$H$ & 1.0 & 5.9964815708 & 5427 & 0.4 \\
\hline $\mathbf{H}$ & 1.0 & 6.0056899779 & -1.7912406136 & 2.17 \\
\hline 11 & 1.0 & 5.0581225814 & -0.4097333224 & 1.5914807905 \\
\hline H & 1.0 & 4.0306214387 & -3.5023697227 & -2.0849652021 \\
\hline $\mathbf{H}$ & 1.0 & 5.537 & -2.896 & -1.3 \\
\hline U & 1.0 & 4.9194944450 & -2.13 & -2.84 \\
\hline $\mathrm{H}$ & 1.0 & -1.5275525705 & -5.4979886670 & -1.6168936215 \\
\hline 11 & 1.0 & -2.4660045267 & -4.0461970755 & -1.2266395839 \\
\hline $\mathrm{H}$ & 1.0 & -2.0564612058 & -5.2092540676 & 0.0513308553 \\
\hline $\mathbf{H}$ & 1.0 & -0.7634036408 & -4.3227668802 & 2.0342808000 \\
\hline $\mathrm{H}$ & 1.0 & -2.3964968945 & -4.8904338957 & 2.4372861533 \\
\hline & 0 & 279287 & -3.7141847632 & 3.48 \\
\hline
\end{tabular}




\begin{tabular}{|c|c|c|c|}
\hline & $\begin{array}{ll}.0 & -3.3974592695\end{array}$ & 1.2062584430 & -0.70077 \\
\hline & $1.0-3.5558018996$ & -0.1843123490 & -2.819972672 \\
\hline & $.0 \quad-5.0976434347$ & -0.9134580553 & -3.29508411 \\
\hline & $\begin{array}{ll}1.0 & -4.1223635178\end{array}$ & -1.6732082153 & -2.03427729 \\
\hline & $1.0-7.0912619763$ & -0.8551309935 & -1.693377145 \\
\hline & $1.0-6.9305560694$ & -0.1039208813 & -0.099420 \\
\hline & $1.0-6.0870869548$ & -1.6237162820 & -0.457418312 \\
\hline & $1.0-6.3497620632$ & 1.1865780224 & -2.898757643 \\
\hline & $1.0-4.8430791367$ & 2.0100893120 & -2.4632211652 \\
\hline & $1.0-6.2307397067$ & 2.0527655168 & -1.359717611 \\
\hline & $1.0-4.0896018871$ & 3.1416299490 & 0.5261962523 \\
\hline & $1.0-3.2606109610$ & 2.0915259553 & 1.7189012862 \\
\hline & $1.0-4.9214447158$ & 2.7092770284 & 2.0604739750 \\
\hline & $1.0 \quad 0.8299047851$ & 3.1129421928 & 37060 \\
\hline & $1.0 \quad 2.4306596283$ & 2.8941731198 & -2.5513824301 \\
\hline & $1.0 \quad 2.5798602685$ & 1.4252946788 & -4.6077952871 \\
\hline & $1.0 \quad 3.1890786213$ & -0.2618883414 & -4.478034377 \\
\hline & $1.0 \quad 3.7787904936$ & 0.9984007695 & -3.3431 \\
\hline & $1.0 \quad 4.0045660051$ & 2.6306870071 & -0.6966921356 \\
\hline & $1.0 \quad 3.7050117276$ & 0.9097396169 & -0.2871263776 \\
\hline & $1.0-0.0238466344$ & 3.2064783149 & 1.6996217151 \\
\hline & $1.0 \quad 3.4148565039$ & 4.4560740178 & -0.6088257913 \\
\hline & $1.0-0.4786051803$ & 5.5764636395 & 2.2836372861 \\
\hline & $1.0 \quad 2.9256874538$ & 6.8412082917 & -0.0378191805 \\
\hline & $1.0 \quad 0.9819444063$ & 7.3940403480 & 1.414867936 \\
\hline & $1.0 \quad 5.3614613324$ & 1.9411810150 & 1.2832896472 \\
\hline & $1.0 \quad 3.9015149975$ & 1.4588970996 & 2.1661051161 \\
\hline & $1.0 \quad 4.1795799218$ & 3.1663433180 & 1.7745350 \\
\hline
\end{tabular}

--- OPTIMIZED RHF MO-S --- GENERATED AT 11:11:08 LT 8-SEP-2004 $\mathrm{E}=\quad-298.9081110936, \mathrm{E}(\mathrm{NUC})=3616.2024299504$ MODE 1 FREQUENCY $=-392.78428\left(\mathrm{CM}^{* *}-1\right)$ 


\section{Equatorial-Axial isomer, Position $2 b$}

\section{COORDINATES OF SYMMETRY UNIQUE ATOMS (ANGS)}

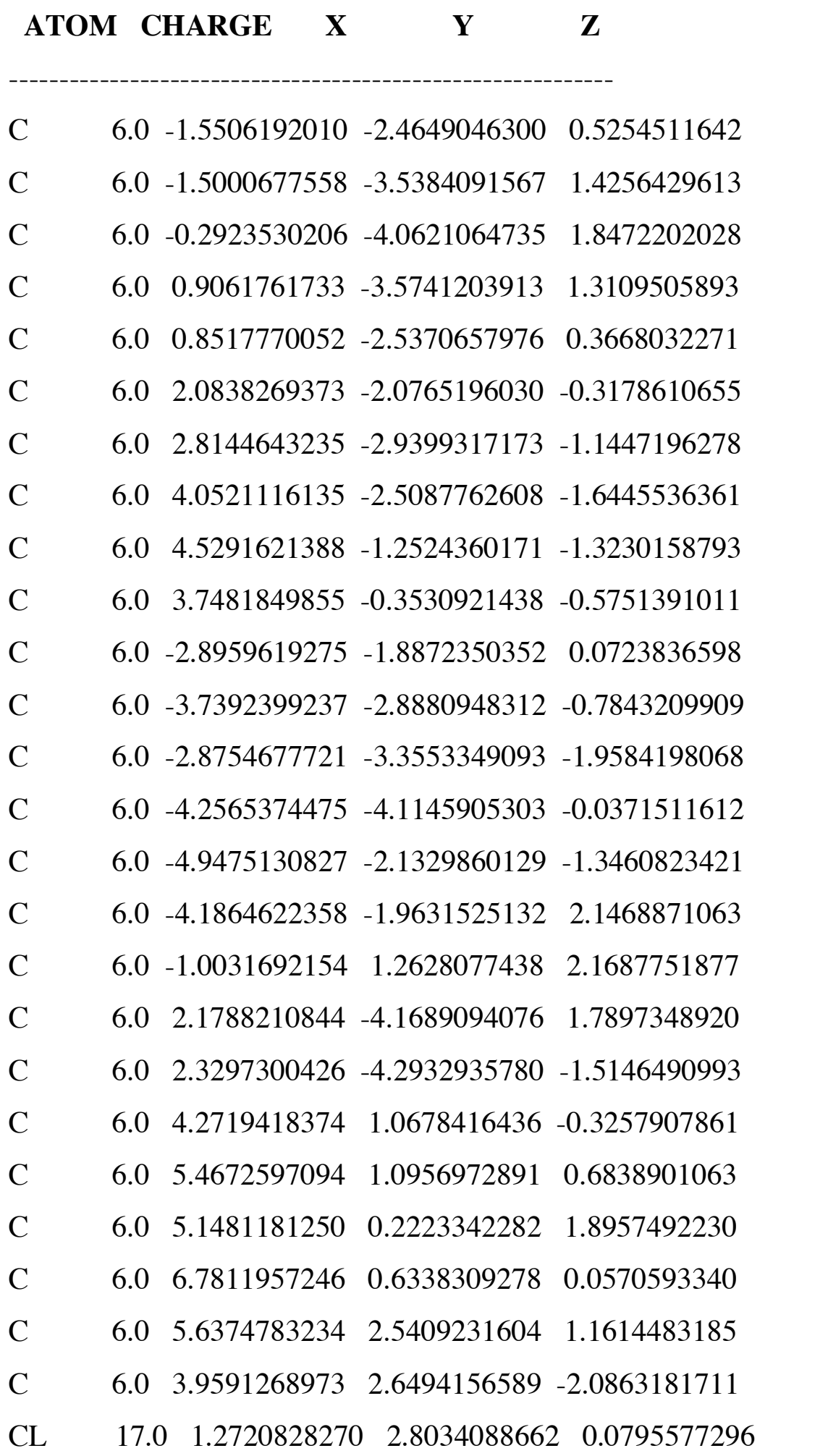




\begin{tabular}{|c|c|c|c|}
\hline $\mathrm{O}$ & $8.0-0.1315453051$ & 0.5478451865 & 1.4972263529 \\
\hline $\mathrm{N}$ & $7.0-0.3654365123$ & -1.9156639069 & 0.0778234002 \\
\hline $\mathrm{N}$ & $7.0 \quad 2.5103339264$ & -0.7578089312 & -0.1340450852 \\
\hline $\mathrm{O}$ & $8.0-0.3576377396$ & -0.8167382953 & -0.6845710799 \\
\hline $\mathrm{O}$ & $8.0 \quad 1.6916723529$ & 0.0734840923 & 0.5478482301 \\
\hline $\mathrm{O}$ & $8.0 \quad-3.5444197194$ & -1.2189560554 & 1.1413343346 \\
\hline $\mathrm{O}$ & $8.0 \quad 4.7074463432$ & 1.5639138018 & -1.5903799354 \\
\hline SI & $14.0 \quad 0.2478651459$ & 0.8099823966 & -0.1815330019 \\
\hline $\mathrm{O}$ & $8.0-0.2568780381$ & 1.8869538967 & 3.1084494071 \\
\hline $\mathrm{C}$ & $6.0-2.3500808713$ & 1.3690524099 & 1.8875691439 \\
\hline $\mathrm{C}$ & $6.0-0.9145542090$ & 2.7478675421 & 4.0145024976 \\
\hline $\mathrm{CL}$ & $17.0 \quad 0.8989719424$ & $4 \quad 0.8134136421$ & -2.3244759083 \\
\hline $\mathrm{C}$ & $6.0-2.1368676094$ & 2.3311426467 & -0.1421270072 \\
\hline $\mathrm{C}$ & $6.0-3.5523379607$ & 2.1912263258 & -0.5872475382 \\
\hline $\mathrm{C}$ & $6.0-1.7315760840$ & 3.7016993402 & 0.3658417461 \\
\hline $\mathrm{C}$ & $6.0-3.8944742693$ & 1.3979246121 & -1.6867317419 \\
\hline $\mathrm{C}$ & $6.0-4.5546292472$ & 2.9122894618 & 0.0745928767 \\
\hline $\mathrm{C}$ & $6.0-5.2143132009$ & 1.3464577983 & -2.1236144101 \\
\hline $\mathrm{C}$ & $6.0-5.8719687561$ & 2.8441283152 & -0.3552927233 \\
\hline $\mathrm{C}$ & $6.0-6.2034252032$ & 2.0645504691 & -1.4605370176 \\
\hline $\mathrm{O}$ & $8.0-1.2955215127$ & 1.4991561703 & -0.6326139254 \\
\hline $\mathrm{C}$ & $6.0-1.4320794635$ & 4.6284572646 & -0.7948982558 \\
\hline $\mathrm{H}$ & $1.0-2.4600960492$ & -3.9433999961 & 1.8024463512 \\
\hline $\mathrm{H}$ & $1.0-0.2668167853$ & -4.8667388070 & 2.5942380263 \\
\hline $\mathrm{H}$ & $1.0 \quad 4.6419984408$ & -3.1759489275 & -2.2874556278 \\
\hline $\mathrm{H}$ & $1.0 \quad 5.5328292114$ & -0.9205200211 & -1.6479458834 \\
\hline $\mathrm{H}$ & $1.0-2.7287136126$ & -0.9807104233 & -0.5751181125 \\
\hline $\mathrm{H}$ & $\begin{array}{ll}1.0 & -3.4571745209\end{array}$ & -3.9790673917 & -2.6514041732 \\
\hline $\mathrm{H}$ & $\begin{array}{ll}1.0 & -2.0224976546\end{array}$ & -3.9578966775 & -1.6188390847 \\
\hline $\mathrm{H}$ & $1.0-2.4821172631$ & -2.5078836444 & -2.5364300803 \\
\hline $\mathrm{H}$ & $1.0-4.7064245980$ & -4.8344321068 & -0.7346251705 \\
\hline
\end{tabular}




\begin{tabular}{|c|c|c|c|c|}
\hline & .0 & -5.0333847420 & -3.8445271150 & 0.69 \\
\hline $\mathrm{H}$ & 1.0 & -3.4590633491 & -4.6414993633 & 0.5088409 \\
\hline $\mathrm{H}$ & 1.0 & -5.6127859936 & -1.7805491901 & -0.545900905 \\
\hline & .0 & -5.5409474972 & -2.7796440687 & -2.00650701 \\
\hline & .0 & -4.6433780238 & -1.2518378739 & -1.93224229 \\
\hline & 1.0 & -3.5330451000 & -2.7300061029 & 2.589532370 \\
\hline & 1.0 & -5.0996046297 & -2.4472509243 & 1.76 \\
\hline & 1.0 & -4.4348702641 & -1.1971739638 & 2.8 \\
\hline F & 1.0 & 2.2297144139 & -4.1278767251 & 2.88 \\
\hline & 1.0 & 3.0684377453 & -3.6576706545 & 1.397788468 \\
\hline & 1.0 & 2.2472792017 & -5.2270666233 & 5655105 \\
\hline & 1.0 & 1.2383349519 & -4.3845548699 & -1.433946487 \\
\hline & 1.0 & 2.7769926830 & -5.0549897551 & -0.8577778021 \\
\hline $\mathrm{H}$ & 1.0 & 2.6071394557 & -4.5433908694 & -2.548555402 \\
\hline & 1.0 & 3.4496065224 & 1.7129733497 & 0.1006 \\
\hline & 1.0 & 4.1935175423 & 0.5057984523 & 2.3590450 \\
\hline & 1.0 & 5.9263333367 & 0.3211807823 & 2.6651412356 \\
\hline & 1.0 & 5.0928436066 & -0.8413143580 & 1.6276082194 \\
\hline & 1.0 & 7.5675891799 & 0.5374301644 & 0.8176 \\
\hline & 1.0 & 7.1412369089 & 1.3457732554 & -0.698 \\
\hline F & 1.0 & 6.6787276380 & -0.3441720951 & -0.4398446979 \\
\hline I & 1.0 & 6.5004929974 & 2.6324166236 & 1.8344385732 \\
\hline & 1.0 & 4.7527866086 & 2.8942424612 & 1.7087722680 \\
\hline & 1.0 & 5.8020863818 & 3.2260979572 & 0.3184830220 \\
\hline I & 1.0 & 4.1095786505 & 3.5455682114 & -1.4726727368 \\
\hline F & 1.0 & 2.8838182926 & 2.4245056336 & -2.1564704634 \\
\hline & 1.0 & 4.3887224247 & 2.7946396427 & -3.0804905370 \\
\hline & 1.0 & -3.0002604690 & 1.9914439627 & 2.4990243209 \\
\hline & 1.0 & -2.8830522980 & 0.5309454869 & 1.407475092 \\
\hline & 1.0 & -1.3633069970 & 3.6041328457 & 3.4964379 \\
\hline & 1.0 & -0.1004234324 & 3.0804147790 & 4.663497 \\
\hline
\end{tabular}



H $\quad \begin{array}{llll}1.0 & -1.6797993726 & 2.2240601112 & 4.5980402005\end{array}$
H $\quad \begin{array}{lllll}1.0 & -2.5615875900 & 4.1054711104 & 0.9897267797\end{array}$
$\mathrm{H} \quad \begin{array}{lllll}1.0 & -0.8428490214 & 3.6452090698 & 1.0316370621\end{array}$
$\mathrm{H} \quad 1.0 \quad-3.1242739705 \quad 0.8253506780 \quad-2.2211526118$
$\mathrm{H} \quad \begin{array}{lllll}1.0 & -4.2758142055 & 3.5417669062 & 0.9349342730\end{array}$
$\mathrm{H} \quad 1.0 \quad-5.4730751642 \quad 0.7413169963 \quad-3.0004870173$
H $\quad \begin{array}{lllll}1.0 & -6.6486739638 & 3.4110738801 & 0.1698330202\end{array}$
$\mathrm{H} \quad \begin{array}{lllll}1.0 & -7.2413094500 & 2.0213539298 & -1.8094445480\end{array}$
$\mathrm{H} \quad 1.0 \quad-1.2754942088 \quad 5.6533946405 \quad-0.4352100358$
$\mathrm{H} \quad \begin{array}{lllll}1.0 & -2.2510712110 & 4.6548312153 & -1.5251126261\end{array}$
$\mathrm{H} \quad \begin{array}{lllll} & 1.0 & -0.5142113141 & 4.3197564277 & -1.3176082570\end{array}$

--- OPTIMIZED RHF MO-S --- GENERATED AT 12:16:07 LT 8-SEP-2004

$\mathrm{E}=\quad-298.9044362330, \mathrm{E}(\mathrm{NUC})=3614.0294338474$

MODE 1 FREQUENCY $=-375.01818\left(\mathrm{CM}^{* *}-1\right)$ 


\section{References}

(1) Denmark, S .E.; Winter, S. B. D.; Xu, X.; Wong, K. T.; J. Am. Chem. Soc. 1996, 118, 7404.

(2) Iwai, I.; Okajima, Y. Chem. Abs. 1959, 5191.

(3) Bolm, C.; Ewald, M.; Felder, M.; Schlingloff, G. Chem. Ber. 1992, 125, 1169.

(4) (a) Dongala, E. B.; Dull, D. L.; Mioskowski, C.; Solladie, G. Tetrahedron Lett. 1973, 14, 4983; (b) Basavaiah, D.; Bharathi, T. K. Tetrahedron Lett. 1991, 32, 3417; (c) Mitsui, S; Imaizumi, S.; Senda, Y.; Konno, K. Chem. Ind. 1964, 233.

Correlation diagram for determining the absolute configuration of product $3 \mathbf{a}$ from reference $4 \mathrm{c}$ :
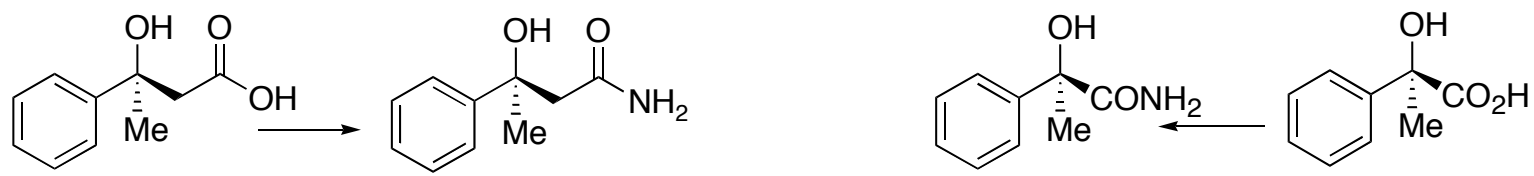

(S) $-(+),+9.86$ in $\mathrm{EtOH}$

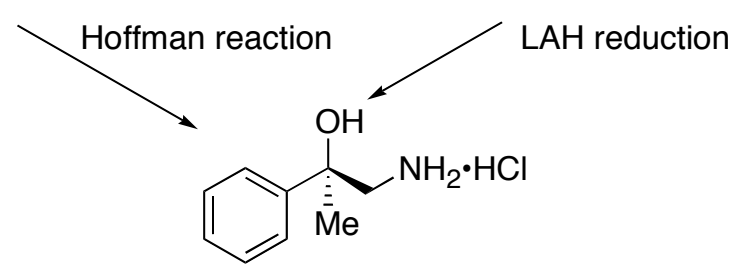

D, $(R)-(-)$ altrolactic acid

$(R)-(-)$

In reference $4 \mathrm{a}$, the rotation of both the methyl ester and its corresponding carboxylic acid were reported. The rotation for product $3 \mathbf{a}$ is $-5.62(\mathrm{c}=1.09$, EtOH). Therefore, its absolute configuration is $S$.<smiles>COC(=O)C[C@@H](O)c1ccccc1</smiles>

$3 a$

(S)-(-), -4.96 in $\mathrm{EtOH}, 58 \%$ ee ref. $4 a$<smiles>C[C@@](O)(CC(=O)O)c1ccccc1</smiles>

(S)-(+), 6.0 in $\mathrm{EtOH}, 58 \%$ ee ref. $4 a$<smiles>COC(=O)C[C@@](C)(O)c1ccccc1</smiles>

obtained value -5.62 in $\mathrm{EtOH}$ $82 \%$ ee determined by SFC $(S)-(-)$

(5) Mitsui, S.; Kudo, Y. Chem. Ind. 1965, 381. 
Correlation diagram for determining the absolute configuration of $\mathbf{3 b}$ from reference 5:

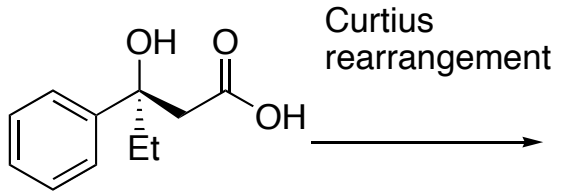

$(S)-(+),+22.0$ in $\mathrm{EtOH}$

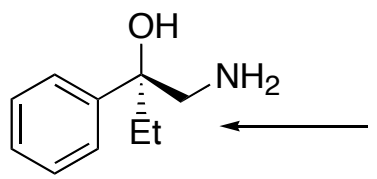

$(S)-(-)$<smiles>CCC(O)(C(N)=O)c1ccccc1</smiles>

(R)-(-)

The absolute configuration of $\mathbf{A}$ was established by the following correlation:<smiles>CCOC(=O)C(O)(CC)c1ccccc1</smiles>

(S)-(+)<smiles>CCC(O)(CCO)c1ccccc1</smiles>

(S)-(-)<smiles>CCC(O)(C[O+])c1cccc(C)c1</smiles><smiles>CI</smiles>

A<smiles>C/C=C\CC(=O)OCC(=O)O</smiles>

(S)-(+)<smiles>CCOCC[C@](C)(O)c1ccccc1</smiles><smiles>[M]C(O)c1ccccc1</smiles>

(S)-(-)

The rotation for the carboxylic acid obtained from the hydrolysis of product $\mathbf{3 b}$ is $+16.0(\mathbf{c}=0.69$, EtOH) (86\% ee determined by SFC). Therefore, the absolute configuration of product $\mathbf{3 b}$ is $S$.

\section{Full References from manuscript}

(10b) Judge, T. M.; Phillips, G.; Morris, J. K.; Lovasz, K. D.; Romines, K. R.; Luke, G. P.; Tulinsky, J.; Tustin, J. M.; Chrusciel, R. A.; Dolak, L. A.; Mizsak, S. A.; Watt, W.; Morris, J.; van der Velde, S. L.; Strohbach, J. W.; Gammill, R. B. J. Am. Chem. Soc. 1997, 119, 36273628.

(35) Schmidt, M. W.; Baldridge, K. K.; Boatz, J. A.; Elbert, S. T.; Gordon, M. S.; Jensen, J. H.; Koseki, S.; Matsunaga, N.; Nguyen, K. A.; Su, S.; Windus, T. L.; Dupuis, M.; Montgomery, Jr., J. A. J. Comput. Chem. 1993, 14, 1347-1363. 